\title{
Computational Modeling of Radiation Effects on Total Temperature Probes
}

\author{
Jonathan Paul Reardon \\ Thesis submitted to the faculty of the Virginia Polytechnic Institute and State University \\ in partial fulfillment of the requirements for the degree of \\ Master of Science \\ In \\ Aerospace Engineering \\ Joseph A. Schetz, Co-Chair \\ K. Todd Lowe, Co-Chair \\ Walter F. O’Brien, \\ $12 / 4 / 2015$ \\ Blacksburg, VA \\ Keywords: Total Temperature, \\ Thermocouple, Computational Fluid Dynamics, Heat Transfer
}




\title{
Computational Modeling of Radiation Effects on Total Temperature Probes
}

\author{
Jonathan Paul Reardon
}

\begin{abstract}
The requirement for accurate total temperature measurements in gaseous flows was first recognized many years ago by engineers working on the development of superchargers and combustion diagnostics. A standard temperature sensor for high temperature applications was and remains to be the thermocouple. However, this sensor is characterized by errors due to conduction heat transfer from the sensing element, as well as errors associated with the flow over it. In particular in high temperature flows, the sensing element of the thermocouple will be much hotter than its surroundings, leading to radiation heat losses. This in turn will lead to large errors in the temperature indicated by the thermocouple. Because the design and testing of thermocouple sensors can be time consuming and costly due to the many parameters that can be varied and because of the high level of detail attainable from computational studies, the use of advanced computational simulations is ideally suited to the study of thermocouple performance.
\end{abstract}

This work sought to investigate the errors associated with the use of total temperature thermocouple probes and to assess the ability to predict the performance of such probes using coupled fluid-heat transfer simulations. This was done for a wide range of flow temperatures and subsonic velocities. Simulations were undertaken for three total temperature thermocouple probe designs. The first two probes were legacy probes developed by Glawe, Simmons, and Stickney in the 1950's and were used as a validation case since these probes were extensively documented in a National Advisory Committee for Aeronautics (NACA) technical report. The third probe studied was developed at Virginia Tech which was used to investigate conduction errors experimentally. In all cases, the results of the computational simulations were compared to the experimental results to assess their applicability. In the case of the legacy NACA probes, it was shown that the predicted radiation correction compared well with the documented values. This served as a validation of the computational method. Next the procedure was extended to the conduction error case, where the recovery factor, a metric used to relate the total temperature of the flow to the total temperature indicated by the sensor, was compared. Good agreement between the experimental results was found. The effects of radiation were quantified and shown to be small. It was also demonstrated that computational simulations can be used to obtain quantities that are not easily measured experimentally. Specifically, the heat transfer coefficients and the flow through the vented shield were investigated. The heat transfer coefficients were tabulated as Nusselt numbers and were compared to a legacy correlation. It was found that although the legacy correlation underpredicted the Nusselt number, the predicted results did follow the same trend. A new correlation of the same functional form was therefore suggested. Finally, it was found that the mounting strut had a large effect on the internal flow patterns and therefore the heat transfer to the thermocouple. Overall, this work highlights the usefulness of computational simulations in the design and analysis of total temperature thermocouple sensors. 


\section{Acknowledgements}

I would like to take a moment to acknowledge all the people who made this work possible. First, a special thanks to Dr. Schetz and Dr. Lowe, my advisors for allowing me to be a part of this research team. Without their advice and guidance, this work would not have been possible. They have contributed significantly to my academic and professional development. I would also like to thank Dr. O'Brien for serving as a member of my committee.

Next, I would like to recognize Pratt \& Whitney for sponsoring this research and all the members of their team for their input and feedback. Working with these members allowed me a great opportunity to engage in work with practicing engineers from industry.

Finally, I need to thank my family and friends for support throughout the past year and a half. Without it, none of this would have been possible.

Thank you,

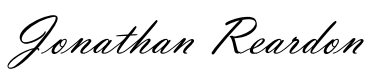




\section{Table of Contents}

List of Figures ........................................................................................................ vi

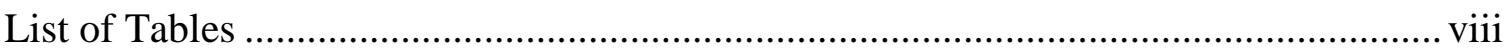

Nomenclature

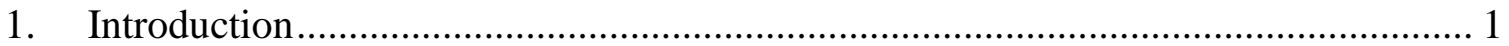

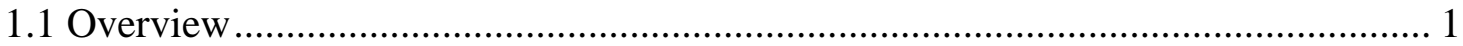

1.2 Review of Thermocouple Sensors and Their Performance ....................................... 3

1.2.1 Thermocouple Types .............................................................................. 3

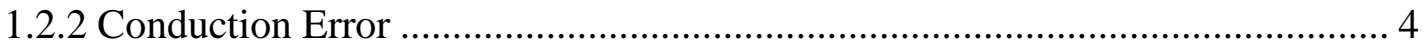

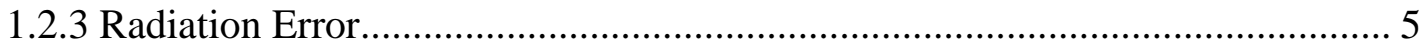

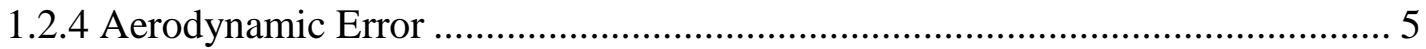

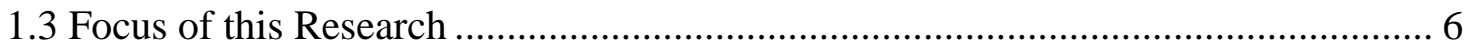

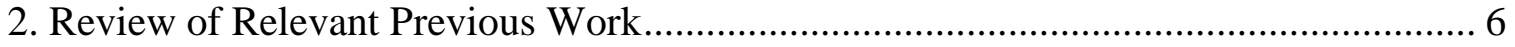

2.1 Work of Glawe, Simmons, and Stickney ............................................................... 6

2.2 Conduction Error Work at Virginia Tech ............................................................ 10

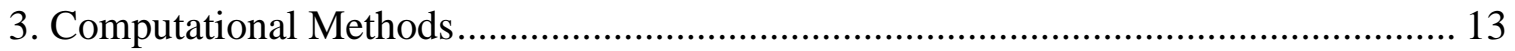

3.1 Governing Equations for Computational Fluid Dynamics ..................................... 14

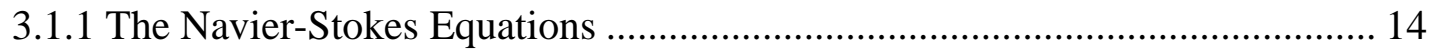

3.1.2 The Reynolds-Averaged Navier-Stokes Equations for Turbulent Flows ........ 14

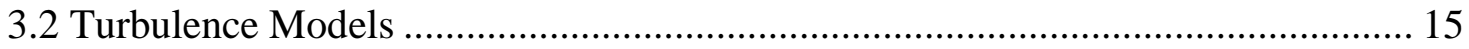

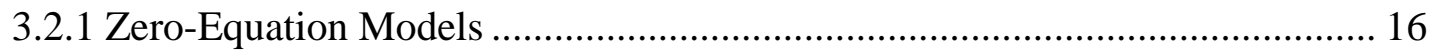

3.2.2 One-Equation Models ……………………….......................................... 16

3.2.3 Two-Equation Models .............................................................................. 16

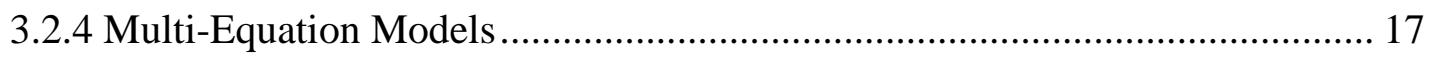

3.3 Conjugate Heat Transfer .............................................................................. 17

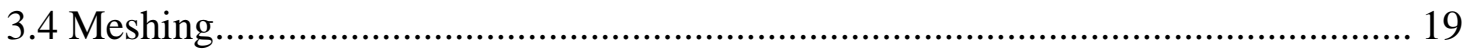

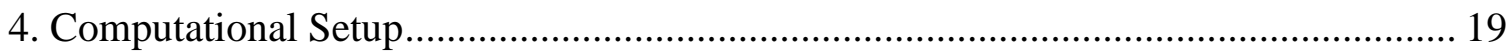

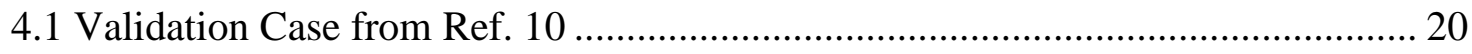

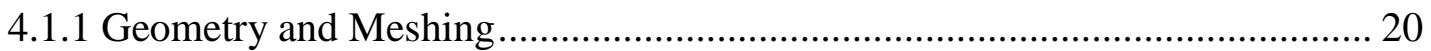

4.1.2 Boundary Conditions and Flow Setup …………………………………..... 23

4.2 Conduction Error Study Test Case from Ref. 14 ............................................. 26

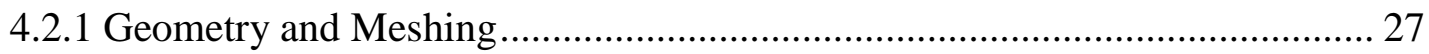

4.2.2 Boundary Conditions and Flow Setup …………….................................... 29

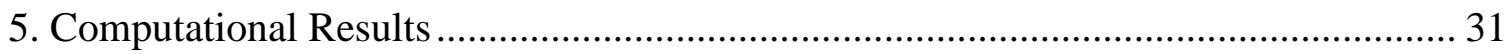




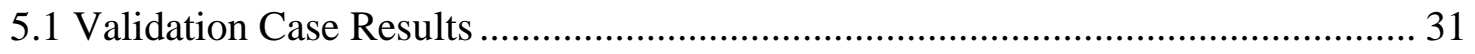

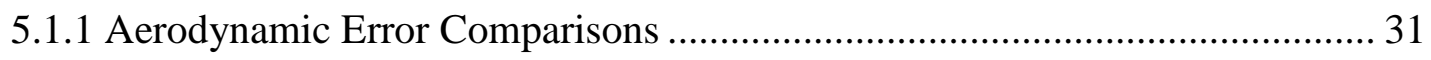

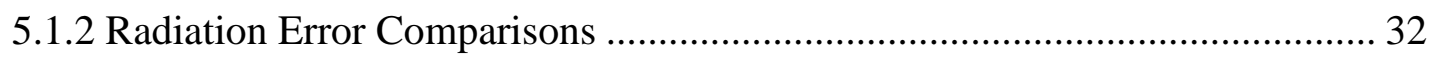

5.2 Conduction Error Test Case Results ................................................................... 38

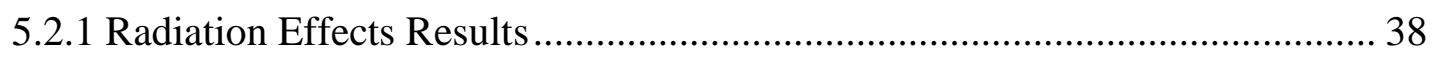

5.2.2 Sensor Recovery Results..................................................................... 41

5.2.3 Convective Heat Transfer Coefficient Results ............................................ 43

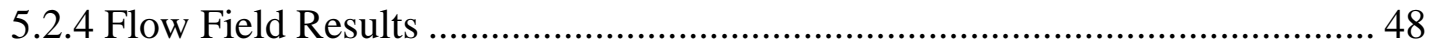

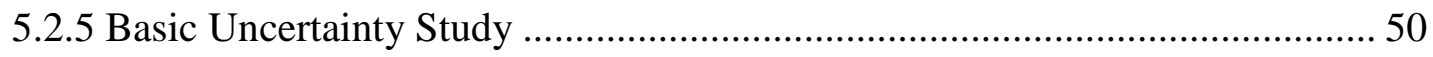

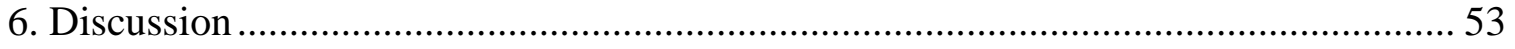

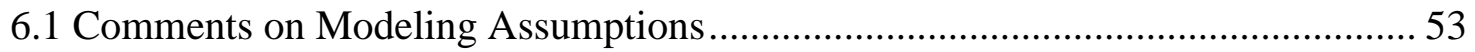

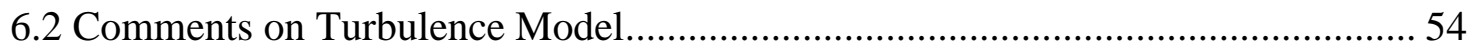

6.3 Comments on the Usefulness of Computational Simulations.............................. 55

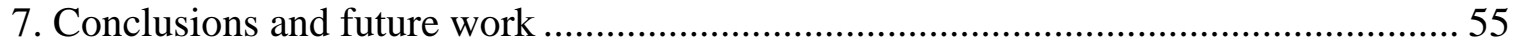

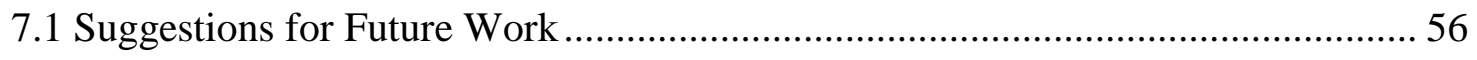

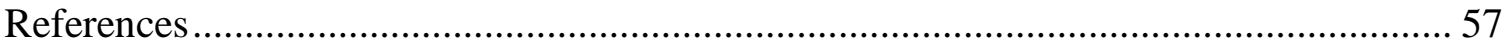




\section{List of Figures}

Figure 1: Schematic of a Thermocouple Loop [2] ................................................. 1

Figure 2: Early Shielded Probe Design [8] ......................................................... 2

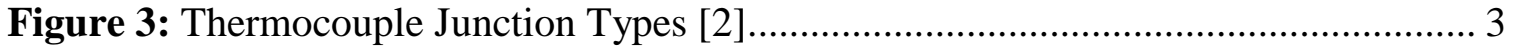

Figure 4: Total Temperature Probes from Ref. 10....................................................... 7

Figure 5: High Temperature Tunnel used in the work of Glawe et al. [10] .................... 8

Figure 6: Variation of the Aerodynamic Recovery Factor at Standard Conditions as a

Function of Mach Number for the Shielded and Unshielded Probes [10]........................ 8

Figure 7: Variation of the Aerodynamic Recovery Factor as a Function of Flow Total

Pressure for the Shielded and Unshielded Probes [10] ................................................. 9

Figure 8: Radiation Correction as a Function of Indicated Junction Temperature for the

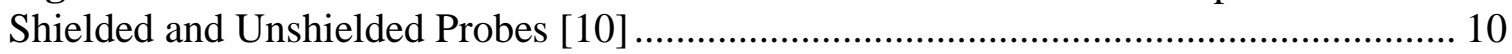

Figure 9: Shielded Probe used in Conduction Error Experiments at Virginia Tech....... 11

Figure 10: Experimental Setup of Virginia Tech Hot Jet Facility ................................. 12

Figure 11: Recovery Results from Thermal Resistance Model from Ref. 14 ................ 13

Figure 12: Computational Domain for Validation Cases .......................................... 20

Figure 13: Three Dimensional Geometry for NACA Shielded Thermocouple Sensor ... 21

Figure 14: Two-Dimensional, Axisymmetric Geometries for the NACA Sensors ......... 21

Figure 15: Unstructured Mesh for Three-Dimensional Model of Shielded Probe [15]... 22

Figure 16: Unstructured Mesh for Two-Dimensional, Axisymmetric Model of Shielded

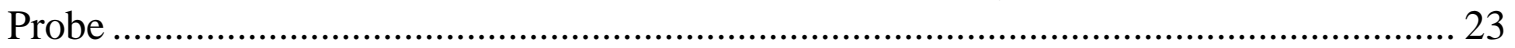

Figure 17: Boundary Conditions for Two-Dimensional, Axisymmetric Simulations ..... 24

Figure 18: Boundary Conditions for Three-Dimensional Simulations......................... 24

Figure 19: Computational Domain for Conduction Error Probe Simulations ................ 27

Figure 20: Three-Dimensional Model of Conduction Error Probe................................. 27

Figure 21: Mesh for Three-Dimensional Conduction Error Probe Simulation [15]....... 28

Figure 22: Boundary Conditions for Conduction Error Test Simulations ...................... 30

Figure 23: Predicted Temperature Distribution for Two-Dimensional, Axisymmetric

Model of Unshielded Probe from Ref. 10 at Flow Total Temperature of 2500R and Mach

Number of 0.3

Figure 24: Predicted Radiative Heat Flux Distribution for Two-Dimensional, Axisymmetric Model of Unshielded Probe from Ref. 10 at Flow Total Temperature of 2500R and Mach Number of 0.3

Figure 25: Predicted Temperature Distribution for Three-Dimensional Model of Shielded Probe of Ref. 10 at Flow Total Temperature of 2500R and Mach Number of 0.3

Figure 26: Predicted Radiative Heat Flux Distribution for Three-Dimensional Model of Shielded Probe of Ref. 10 at Flow Total Temperature of 2500R and Mach Number of 0.3

Figure 27: Radiative Heat Flux from Exposed Thermocouples from the Two-

Dimensional, Axisymmetric Models at Flow Total Temperature of 2500R and Mach

Number of 0.3

Figure 28: Comparison of Radiation Corrections from Current Simulations with Empirical Correlation from Ref. 10 
Figure 29: Radiative Heat Flux Contours for Conduction Error Probe at Total Temperature of $850^{\circ} \mathrm{F}(727 \mathrm{~K})$, Mach Number of 0.8 , and Conduction Driver of $0.5 \ldots . .40$ Figure 30: Radiative Heat Flux Contours along Thermocouple Surface for Conduction Error Probe at Total Temperature of $850^{\circ} \mathrm{F}(727 \mathrm{~K})$, Mach Number of 0.8 , and Conduction Driver of 0.5

Figure 31: Temperature Contours for Conduction Error Probe at Total Temperature of $850^{\circ} \mathrm{F}(727 \mathrm{~K})$, Mach Number of 0.8 , and Conduction Driver of 0.5

Figure 32: Temperature Contours in Sheath and TC Wire for Conduction Error Probe at Total Temperature of $850^{\circ} \mathrm{F}(727 \mathrm{~K})$, Mach Number of 0.8 , and Conduction Driver of 0.5

Figure 33: Recovery as a Function of Mach Number for Conduction Error ProbeExperimental Data [14] and Current Predictions.

Figure 34: Recovery as a Function of Reynolds Number for Conduction Error Probe Experimental Data [14] and Current Predictions.

Figure 35: In-Shield Reference Point used in Heat Transfer Coefficient Calculations ... 44

Figure 36: Contours of Wall Heat Flux along Thermocouple Body for Conduction Error Probe at Total Temperature of $850^{\circ} \mathrm{F}(727 \mathrm{~K})$, Mach Number of 0.8 , and Conduction Driver of 0.5

Figure 37: Contours of Nusselt Number along Thermocouple Body for Conduction Error Probe at Total Temperature of $850^{\circ} \mathrm{F}(727 \mathrm{~K})$, Mach Number of 0.8 , and Conduction

Driver of 0.5 45

Figure 38: Comparison of Average Max Nusselt Numbers from Simulations and Legacy Correlation from Ref. 9

Figure 39: Ratio of In-Tube Velocity to Freestream Velocity for CFD Predictions and

Estimate from Ref. 9 48

Figure 40: Mach Number Contours for Conduction Error Probe at Total Temperature of $850^{\circ} \mathrm{F}(727 \mathrm{~K})$, Mach Number of 0.8 , and Conduction Driver of 0.5

Figure 41: Pressure Contours at Leading Edge of Strut for Conduction Error Probe at Total Temperature of $850^{\circ} \mathrm{F}(727 \mathrm{~K})$, Mach Number of 0.8 , and Conduction Driver of 0.5

Figure 42: Streamlines through Shield for Conduction Error Probe at Total Temperature of $850^{\circ} \mathrm{F}(727 \mathrm{~K})$, Mach Number of 0.8 , and Conduction Driver of 0.5 50

Figure 43: Uncertainty in Radiation Correction for Four Variables of Interest for Conduction Error Probe at Total Temperature of $850^{\circ} \mathrm{F}(727 \mathrm{~K})$, Mach Number of 0.8 , and Conduction Driver of 0.5 


\section{List of Tables}

Table 1: Standard Thermocouple Types [2] ........................................................... 4

Table 2: Calculation of Radiation Quantities in DO Model ........................................ 19

Table 3: Thermo-physical Properties of Thermocouple Components used in Simulations of NACA Probes

Table 4: Equilibrium Mass Fractions for Combustion of 72 Octane with Air used in

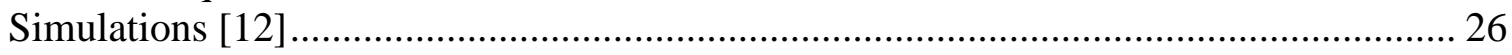

Table 5: Test Matrix for Computational Study of Conduction Error ............................. 29

Table 6: Thermo-Physical Properties of Thermocouple and Strut Components in Conduction Error Simulations 31

Table 7: RMS Error and Percent Difference between Predicted Radiation Corrections and Reported Data from Ref. 10 36

Table 8: Recovery Values from Simulations with and without Radiation Modeling ...... 39 Table 9: Ratio of Convective Heat Transfer Coefficient at Stagnation Point to Average along Body of Thermocouple

Table 10: Input Variables for Radiation Uncertainty Quantification at Total Temperature of $850^{\circ} \mathrm{F}(727 \mathrm{~K})$, Mach Number of 0.8 , and Conduction Driver of 0.5

Table 11: Sensitivity Derivatives for Radiation Uncertainty at Total Temperature of $850^{\circ} \mathrm{F}(727 \mathrm{~K})$, Mach Number of 0.8 , and Conduction Driver of 0.5 52

Table 12: Eddy Viscosity Ratio inside Shield of Conduction Error Probe. 54 


\section{Nomenclature}

$\begin{array}{ll}a & =\text { absorption coefficient } \\ A_{R} & =\text { radiation heat transfer area } \\ A_{C} & =\text { convective heat transfer area } \\ B i & =\text { Biot number } \\ C_{\mathrm{p}} & =\text { specific heat at constant pressure } \\ \mathrm{C}_{\text {radiation }} & =\text { radiation temperature correction } \\ d_{\text {wire }} & =\text { thermocouple wire diameter } \\ E_{\text {velocity }} & =\text { temperature error due to flow velocity } \\ E_{\text {conduction }} & =\text { temperature error due to conduction } \\ E_{\text {radiation }} & =\text { temperature error due to radiation } \\ h & =\text { convective heat transfer coefficient } \\ I & =\text { radiation intensity } \\ k & =\text { turbulent kinetic energy } \\ k_{f} & =\text { fluid thermal conductivity } \\ k_{s} & =\text { solid material thermal conductivity } \\ k_{T} & =\text { turbulent thermal conductivity } \\ k_{e f f} & =\text { effective thermal conductivity } \\ K_{R} & =\text { radiation form factor } \\ K_{\text {rad }}^{*} & =\text { radiation correction coefficient } \\ L & =\text { thermocouple wire length } \\ l_{m} & =\text { mixing length } \\ M & =\text { Mach number } \\ M W_{i} & =\text { molecular weight of species “i” } \\ n & =\text { refractive index } \\ N u & =\text { Nusselt number } \\ P & =\text { static pressure } \\ P_{t} & =\text { total pressure } \\ P r & =\text { Prandtl Number } \\ P r_{T} & =\text { turbulent Prandtl number } \\ q_{\text {cond }} & =\text { conduction heat transfer } \\ q_{\text {conv }} & =\text { convective heat transfer } \\ q_{\text {rad }} & =\text { radiative heat transfer } \\ R & =\text { overall temperature recovery } \\ \vec{r} & =\text { position vector } \\ R e & =\text { Reynolds number } \\ S & =\text { path length } \\ \vec{s} & =\text { direction vector } \\ \vec{s}^{\prime} & =\text { scattering direction vector } \\ T_{b} & =\text { thermocouple base temperature } \\ T_{d} & =\text { surrounding duct temperature } \\ T_{g} & =\text { gas temperature } \\ T_{s u r r} & =\text { temperature of surroundings } \\ T_{t} & =\text { total temperature } \\ T_{j} & =\text { thermocouple junction temperature } \\ & \end{array}$




$$
\begin{array}{ll}
u_{*} & =\text { friction velocity } \\
x_{i} & =\text { mole fraction of species "i”, } \\
\alpha & =\text { aerodynamic recovery factor } \\
\alpha_{\mathrm{w}} & =\text { absorptivity of a surface } \\
\gamma & =\text { ratio of specific heats } \\
\Delta & =\text { aerodynamic correction factor } \\
\varepsilon & =\text { turbulence dissipation } \\
\varepsilon_{w} & =\text { emissivity of a surface } \\
\theta & =\text { conduction driving potential } \\
v & =\text { kinematic viscosity } \\
v_{T} & =\text { eddy viscosity } \\
\rho & =\text { density } \\
\rho_{w} & =\text { reflectivity of a surface } \\
\sigma & =\text { Stefan-Boltzmann constant } \\
\sigma_{s} & =\text { scattering coefficient } \\
\tau_{T} & =\text { Reynolds shear stress } \\
\tau_{w} & =\text { wall shear stress } \\
\Phi & =\text { phase function } \\
\omega & =\text { turbulence frequency } \\
\Omega & =\text { solid angle }
\end{array}
$$




\section{Introduction}

\subsection{Overview}

The need for accurate high total temperature measurements in jet engines and other applications first developed from the advent of superchargers and other advanced engine components. Jet engines are not only continuing to operate at ever increasing temperatures to maximize efficiency and output, but their size is also being reduced to save weight, leading to much smaller internal passages and cavities. Because of this, having small, accurate total temperature measurement devices is of great value for the determination of efficiency of the engine components as well as for the design and selection of materials to be used within the engine.

The most common temperature sensor for high temperature applications has been and remains to be the thermocouple. Briefly described, a thermocouple is made by joining two dissimilar metal wires and exposing one junction to an unknown temperature while exposing another junction to a constant reference temperature. Based on the Seebeck effect, a voltage difference will develop that can be correlated to the temperature difference. This can then be measured with a voltmeter placed between the two junctions. If one junction is kept at a known temperature, the other junction can be used to measure an unknown temperature. This is shown schematically below in Figure 1. A more in depth discussion of thermocouples will be presented in a subsequent section but the reader is directed to Refs. 1-5 for an overview of temperature sensors and their use.

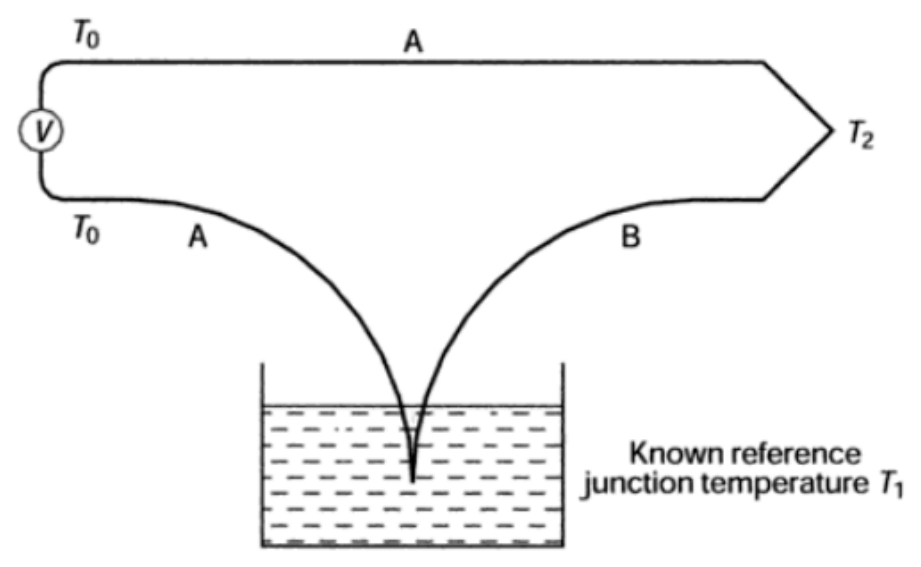

Figure 1: Schematic of a Thermocouple Loop [2]

One design of total temperature sensors which has remained popular and has been used in many fields of study is the shielded thermocouple probe, such as the early example in Figure 2 below, and presented in Refs. 6-8. Although commonly used, this sensor's measurement can be affected by uncertainties due to conduction through instrumentation wires and thermal radiation to the surroundings. In addition, when used in high speed, 
high temperature gaseous flows, the process of stagnating the flow becomes more complicated, which can also lead to errors. The addition of a shield surrounding the sensing element was applied to reduce the flow velocity and thereby the velocity error, as well as to shield the hot junction from the cooler surroundings to reduce the radiation error.

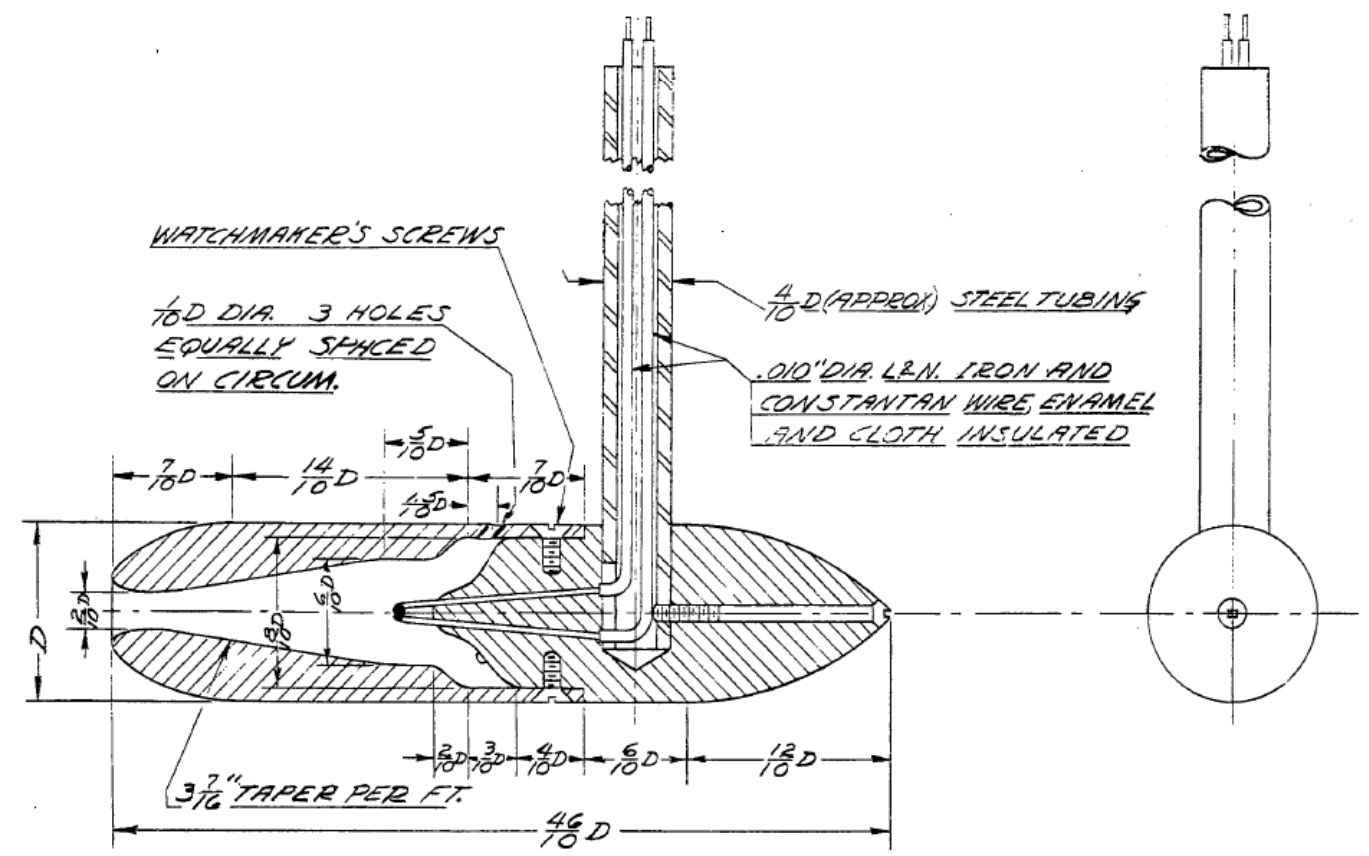

Figure 2: Early Shielded Probe Design [8]

To quantify the impact of these uncertainties, the recovery factor is defined as the ratio of the total temperature indicated by the probe to the true total temperature of the flow, and it is used as a performance metric of the sensor. The recovery factor of a probe can be affected by the conditions of the flow as well as its orientation within the flow. Due to this, design and testing of these probes is both costly and time-consuming. Therefore, simulation, in particular coupled fluid-heat transfer simulations, can be an invaluable tool used in the design of these probes. Simulation allows rapid design iterations to be completed in a relatively short amount of time, allowing designers to better define details of the geometry early in the design process. They also allow the engineer to conduct an in-depth investigation into aspects of the sensor's performance that would be difficult or impossible to investigate experimentally. This is especially true as the size of sensors continues to decrease.

This work utilizes computational simulations to investigate the use of total temperature probes in high subsonic, high temperature flows, with an emphasis on radiation errors. Particularly, this work builds from previous studies that will be reviewed shortly.

First, a review of thermocouples and the major sources of error when used in high temperature, high velocity gaseous flows as studied in historic and recent investigations will be presented. Next, the computational methods used will be described in detail and then applied to a validation case and then to the subjects of the present study at Virginia 
Tech. The results of the computational models will be presented and compared to experimental data to first validate the ability to model radiative heat transfer and then show the ability to predict a sensor's performance. From this, the value of computational modeling in the design of total temperature sensors will be shown.

\subsection{Review of Thermocouple Sensors and Their Performance}

A thermocouple can be formed by connecting two metal wires of different materials [4, 5]. If there is a temperature difference between two junctions, a voltage difference will exist. This is the Seebeck effect and the voltage will be proportional to the temperature difference and will vary depending on the material of the wires used [4,5]. In this work, thermocouples will be discussed with respect to their use in gases although they can be used in other media.

\subsubsection{Thermocouple Types}

There are several types of thermocouples commonly used, and they are classified based on their design and the material combination used in their wires. The two most common types of thermocouples, and the two that will be discussed exclusively in this work, are the bare wire and the sheathed thermocouple. A bare wire thermocouple is one in which the two wires are exposed to the flow as seen in Figure 3A. The wires that form the two legs of the thermocouple are usually welded together to form a small bead. In a sheathed thermocouple (Figure 3B and C) the two thermocouple legs are contained within a sheath and are generally surrounded by a potting material. Sheathed thermocouples can be either grounded or ungrounded indicating that the junction is in contact with the sheath or not, respectively. Also, thermocouples are classified based on the wire combinations. The standard thermocouple types are shown in Table 1 with their general operating temperature range.

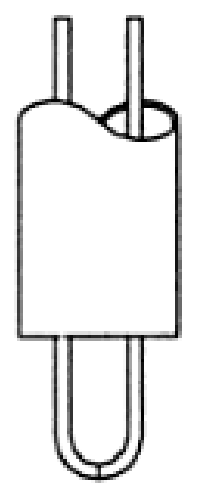

A) Bare Wire

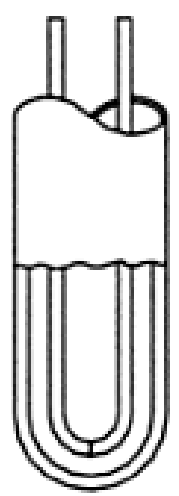

B) Ungrounded, Sheathed

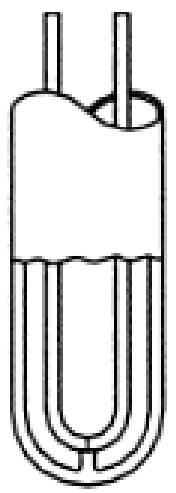

C) Grounded, Sheathed

Figure 3: Thermocouple Junction Types [2] 
Table 1: Standard Thermocouple Types [2]

\begin{tabular}{|c|c|c|}
\hline Type & Material & Temperature Range $\left({ }^{\circ} \mathbf{C}\right)$ \\
\hline T & Copper-Constantan & -262 to 850 \\
\hline J & Iron-Constantan & -196 to 700 \\
\hline E & Chromel-Constantan & -268 to 800 \\
\hline K & Chromel-Alumel & -250 to 1100 \\
\hline N & Nicrosil-Nisil & 0 to 1250 \\
\hline B & $\begin{array}{c}\text { Platinum-30\% Rhodium- } \\
\text { Platinum-6\% Rhodium }\end{array}$ & 100 to 1750 \\
\hline S & $\begin{array}{c}\text { Platinum-10\% Rhodium- } \\
\text { Platinum }\end{array}$ & 0 to 1500 \\
\hline R & $\begin{array}{c}\text { Platinum-13\% Rhodium- } \\
\text { Platinum }\end{array}$ & 0 to 1600 \\
\hline
\end{tabular}

Despite their common use, the temperature measurement by a thermocouple in a gaseous flow can be affected by several errors. It is known that the actual temperature the sensing junction indicates is in fact an equilibrium temperature due to the three modes of heat transfer: conduction, convection, and radiation [9-11]. High relative convective heat transfer from the fluid to the thermocouple junction is the desired condition. However, a large temperature gradient between the junction and the base of the thermocouple can lead to conduction heat transfer existing along the wires (and sheath) of the thermocouple. Cooler surroundings can lead to radiation heat transfer from the thermocouple. Both effects cause the thermocouple to indicate a temperature less than the true total temperature of the gas flow. Also, if the thermocouple is immersed in a gas stream that is at a high velocity, there will be an aerodynamic or velocity error. An in depth derivation of the errors associated with the use of thermocouple sensors in high temperature, high velocity flows can be found in Ref. 13. However, in practice, a reduced form of these equations can be used when it is assumed that each error can be isolated from the others [9]. Those equations as developed in Ref. 9 will be presented here.

\subsubsection{Conduction Error}

Conduction error can be a significant source of uncertainty in thermocouple measurements, especially if the thermocouple is mounted in a fixture that is at a much lower temperature than its exposed junction. If this occurs, a large temperature gradient will exist from the junction to the thermocouple base, leading to significant conduction heat transfer along the thermocouple wires and sheath, if present. The error due to conduction has been derived assuming one dimensional conduction heat transfer analysis and can be shown to be

$$
E_{\text {conduction }}=\frac{T_{t}-T_{b}}{\cosh \left[L\left(4 h / d_{\text {wire }}\right)^{0.5}\right]}
$$


where $T_{t}$ is the total temperature of the flow, $T_{b}$ is the base temperature of the thermocouple, $L$ and $d_{\text {wire }}$ are the length and diameter of the thermocouple wires, respectively, $h$ is the convective heat transfer coefficient, and $k$ is the thermal conductivity of the wires. To reduce this error, it is best to increase the thermocouple's length-to-diameter ratio [9]. This simple expression is mainly applicable to bare wire thermocouples as shown in Figure 3A.

\subsubsection{Radiation Error}

At high temperatures, radiation heat transfer begins to play an increasingly important role. If the temperature of the surroundings is lower than the temperature at the junction of the thermocouple, radiation heat transfer will exist from the junction to the surroundings, reducing the temperature that the thermocouple indicates. The error associated with this radiative exchange can be estimated using

$$
E_{\text {radiation }}=\frac{K_{R} \varepsilon_{w} \sigma A_{R}\left(T_{j}^{4}-T_{\text {surr }}^{4}\right)}{h A_{C}}
$$

where $K_{R}$ is the radiation form factor, $\sigma$ is Stefan-Boltzmann constant, $A_{R}$ is the area on which radiation is experience, $A_{C}$ is the area on which convection acts, and $T_{j}$ and $T_{\text {surr }}$ are the junction and surrounding temperatures of the thermocouple, respectively. The term $K_{R}$ depends upon many factors in a complicated manner.

\subsubsection{Aerodynamic Error}

Aerodynamic or velocity error in the measurement of a total temperature thermocouple exists when the probe is immersed in a high velocity gas stream. This error is due to losses as the flow is slowed to near stagnation values on the sensing element. The total temperature of a fluid is the sum of the static temperature and the kinetic energy associated with the bulk motion of the fluid. As the flow is stagnated, that is brought to rest, the kinetic energy is converted to static temperature. However, due to effects within the viscous boundary layer that wets the surface of the thermocouple, not all of the kinetic energy is returned to static temperature. Instead, some of this energy is redistributed throughout the boundary layer. This in turn reduces the temperature indicated by the thermocouple to less than the actual flow total temperature, leading to error. Because this source of error is due to the incomplete conversion of kinetic energy, it will increase as the flow velocity increases. This is the reason for shielded probes with vent holes, that is, to reduce the flow velocity to a fraction of that of the freestream over the sensing element. The aerodynamic or velocity error can be calculated as

$$
E_{\text {velocity }}=(1-\alpha) \frac{[(\gamma-1) / 2] M^{2}}{1+[(\gamma-1) / 2] M^{2}} T_{t}
$$

where $\alpha$ is the aerodynamic recovery factor which is dependent on the flow properties and the geometry. From the work of Moffat, the recovery factor was found to be 
$\alpha=0.68 \pm 0.07$ and $\alpha=0.86 \pm 0.09$ for bare wire thermocouples perpendicular and parallel to the flow, respectively [9].

\subsection{Focus of this Research}

Total temperature probes are used in a wide variety of applications and in many different fields of study. The general analysis and study of temperature sensor performance is equally broad. This work seeks to focus specifically on hot flows in the high subsonic flow regime indicative of flows encountered in jet engine testing. In particular, this work seeks to build upon recent conduction error studies performed at Virginia Tech to include effects of radiation for the analysis of probes at higher temperatures. To do this, multiphysics computational simulations were utilized to calculate simultaneously the flow over and heat transfer to and from the total temperature probes.

\section{Review of Relevant Previous Work}

Again, the general topic of thermocouple performance is very broad and has been studied and documented extensively. The reader is directed to books on the subject such as Refs. 1-5 and historical reports and review articles such as Refs. 7-11 for a comprehensive review of works to date. The goal of this section is not to present this review, but to highlight the major works that contributed directly to the current study. First, a case chosen to be used as validation for the computational results will be described. Second, recent work that focused on the effect of conduction errors and from which the current study developed will be highlighted.

\subsection{Work of Glawe, Simmons, and Stickney}

In the 1950's Glawe, Simmons, and Stickney [10] investigated the radiation and aerodynamic errors as well as the time constants of a variety of thermocouple probe designs. Because of the extensive documentation and relevant flow conditions considered, this work was chosen as the validation case for the computational simulations to be presented in the current study. For the validation, two probes from this work were chosen in particular. These were the shielded and unshielded probes shown in Figure 4 oriented such that the gas flow would be from left to right in the figure. Both probes use a 20-gage (0.032 inch diameter) Chromel-Alumel (Type K) bare-wire thermocouple. Also, a 3/16 inch outer diameter of sheathing, constructed of Inconel, was used around the thermocouple wires with Magnesium Oxide potting. The shield, when used, was also made of Inconel and was 1/4 inches in outer diameter with eight 0.040 inch vent holes located 3/8 inch from the leading edge of the shield. All of the probes studied were designed with large length-to-diameter ratios to minimize errors due to conduction [10]. In addition, as will be shown, the two probes chosen in particular had small aerodynamic errors at low velocities. This allowed for a detailed investigation into radiation errors at low velocities where aerodynamic errors were negligible, as well as a detailed investigation into aerodynamic errors at low temperatures where radiation errors were negligible, in both cases knowing again that conduction errors could be neglected. 


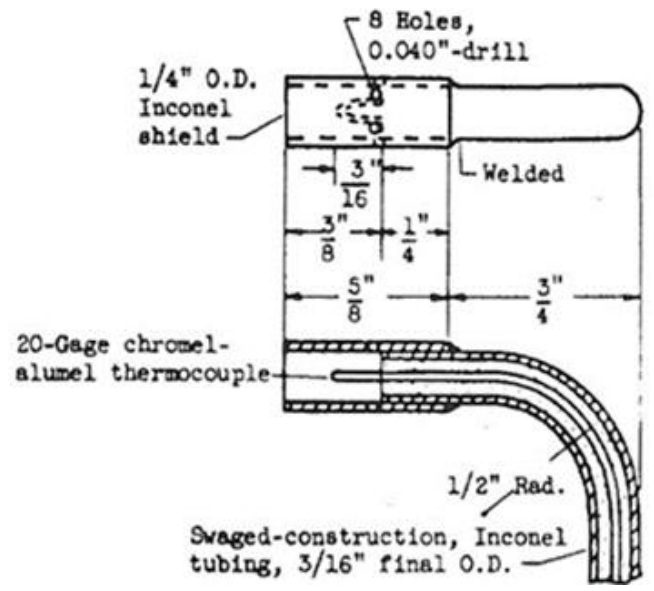

A) Shielded

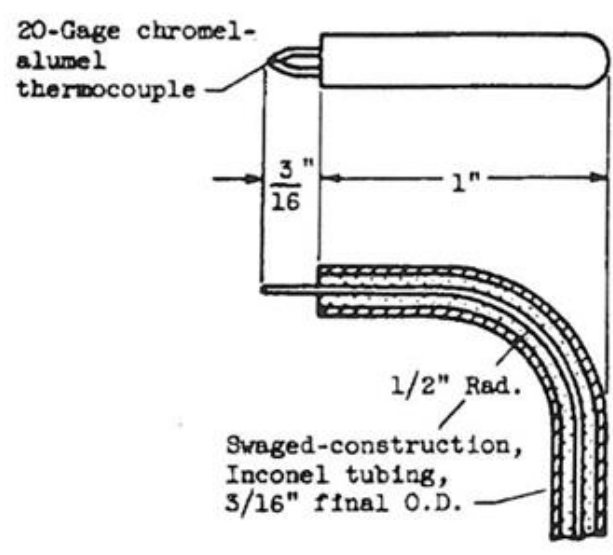

B) Unshielded

Figure 4: Total Temperature Probes from Ref. 10

To conduct these tests, a high temperature tunnel, shown in Figure 5, was used. The test section was located downstream of the combustor section and fed by a nozzle. The probes under test could be aligned along the centerline of the nozzle and retracted via the actuators. This allowed for the transient testing.

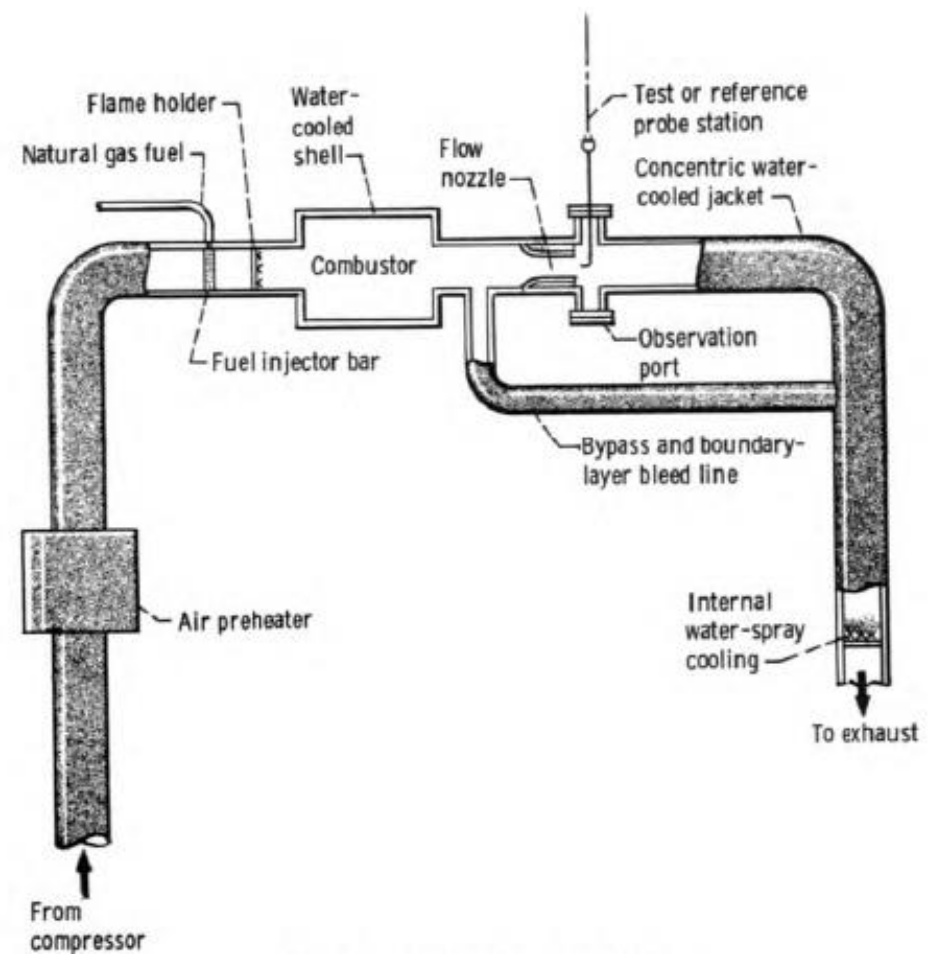

A) Tunnel Schematic 


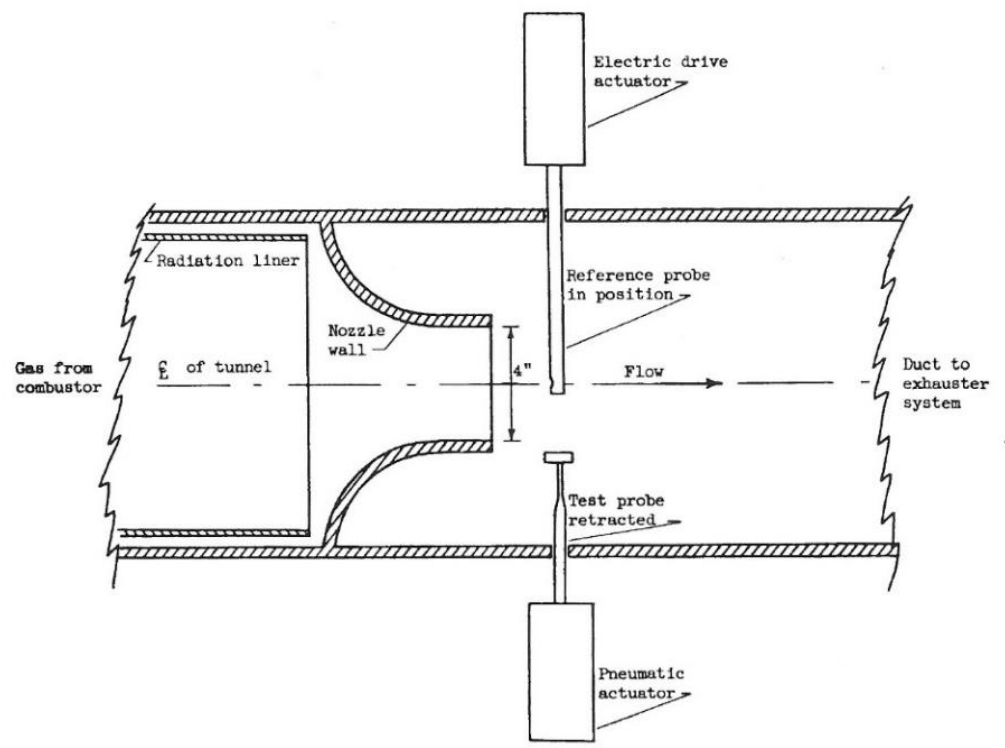

B) Enlarged View of Test Section of High Temperature Tunnel

Figure 5: High Temperature Tunnel used in the work of Glawe et al. [10]

First, Glawe and co-workers [10] investigated the aerodynamic or velocity errors in the probes. This velocity error was given by them as the aerodynamic recover correction factor, defined as $\Delta=\left(T_{t}-T_{j}\right) / T_{t}$. This factor varies with Mach number and Reynolds number. The variation of the aerodynamic recovery factor at standard temperature and pressure for the shielded and unshielded probes is shown in Figure 6 where $\Delta_{\mathrm{o}}$ indicates standard conditions $\left(\mathrm{T}_{\mathrm{t}}=300 \mathrm{~K}\right.$ and $\left.\mathrm{P}_{\mathrm{t}}=1 \mathrm{~atm}\right)$. Also by changing the total pressure of the flow, the Reynolds number effect can be seen. This is shown in Figure 7.

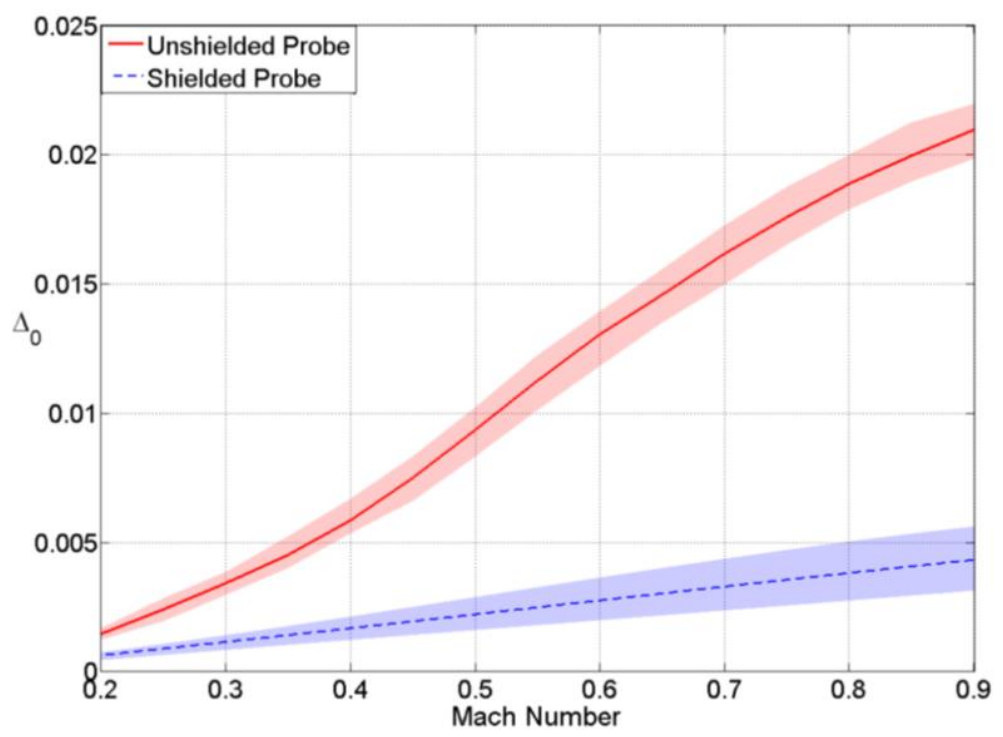

Figure 6: Variation of the Aerodynamic Recovery Factor at Standard Conditions as a Function of Mach Number for the Shielded and Unshielded Probes [10] 


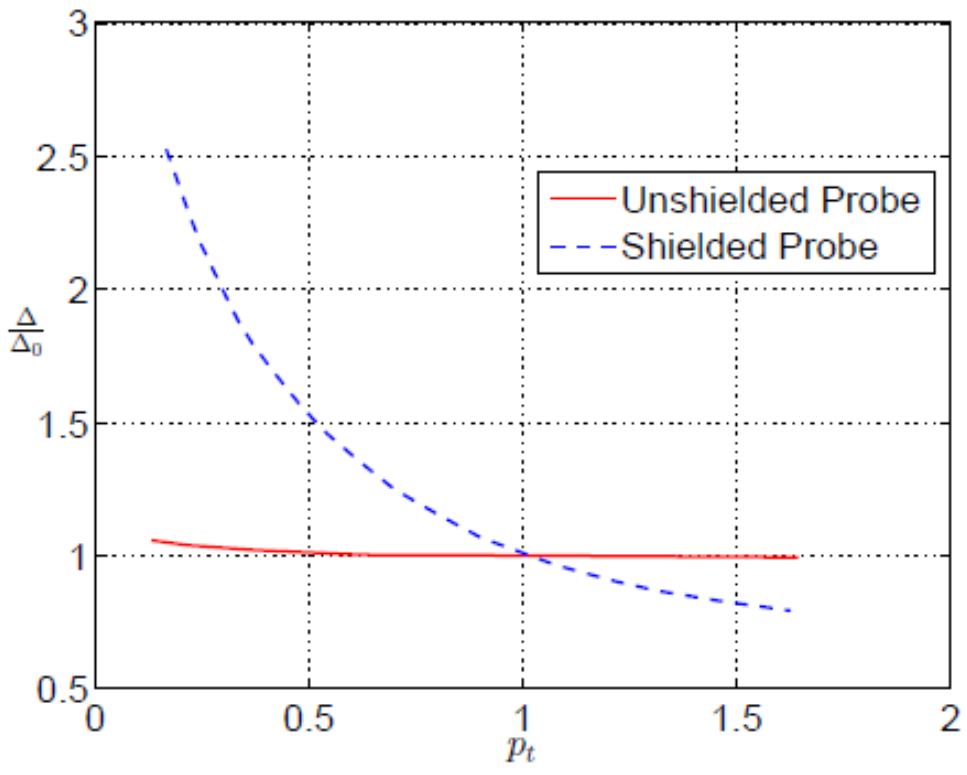

Figure 7: Variation of the Aerodynamic Recovery Factor as a Function of Flow Total Pressure for the Shielded and Unshielded Probes [10]

They also characterized the radiation errors of these two probes by adapting the radiation correction in Ref. 13 as shown below. The radiation corrections are plotted as a function of the indicated junction temperature in Figure 8. Because the effects of conduction were negligible in this setup, the use of the aerodynamic correction and radiation correction could be combined to obtain a simple equation to obtain the true total temperature of the flow given an indicated temperature from the thermocouple, as seen in Eq. 5.

$$
\begin{aligned}
& C_{\text {radiation }}=\frac{K_{r a d}^{*}}{\sqrt{M P}}\left(\frac{T_{j}}{1000}\right)^{-0.18}\left[\left(\frac{T_{j}}{1000}\right)^{4}-\left(\frac{T_{d}}{1000}\right)^{4}\right] \\
& T_{t}=T_{j}+\left\{\frac{K_{r a d}^{*}}{\sqrt{M P}}\left(\frac{T_{j}}{1000}\right)^{-0.18}\left[\left(\frac{T_{j}}{1000}\right)^{4}-\left(\frac{T_{d}}{1000}\right)^{4}\right]\right\}+T_{j} \Delta
\end{aligned}
$$




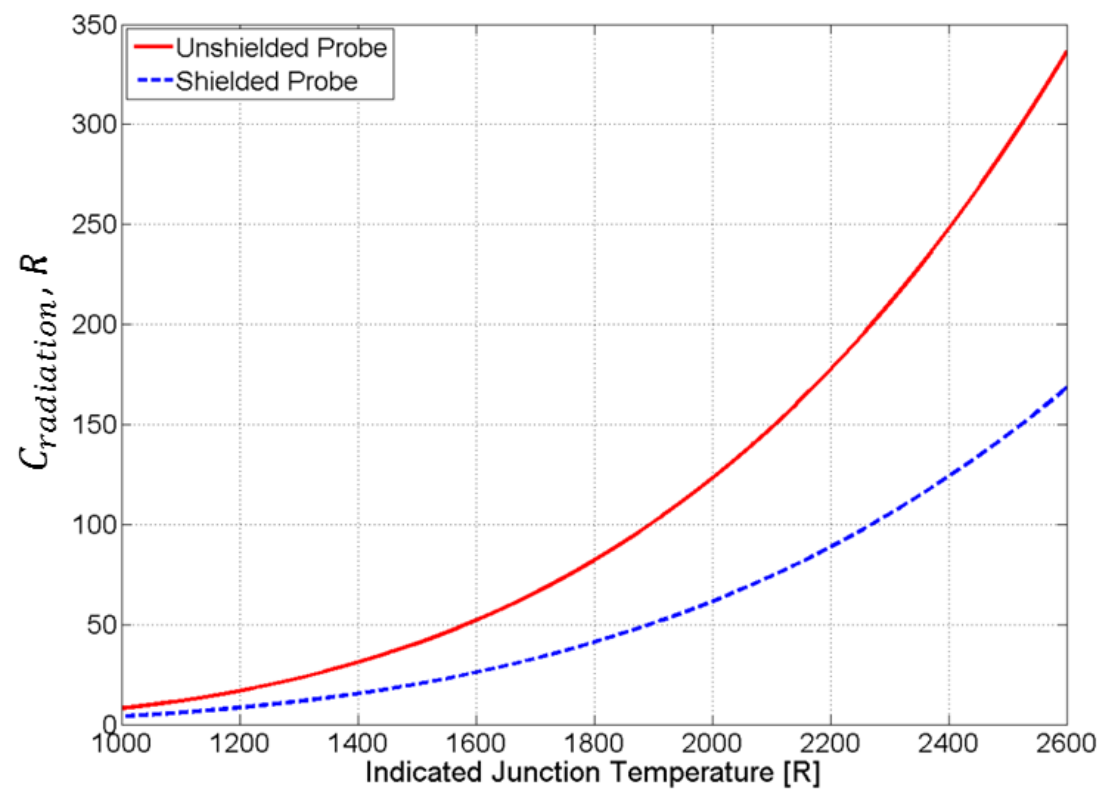

Figure 8: Radiation Correction as a Function of Indicated Junction Temperature for the Shielded and Unshielded Probes [10]

\subsection{Conduction Error Work at Virginia Tech}

Prior to the current study which focuses on radiation error, a comprehensive study of conduction error was performed at Virginia Tech both experimentally, as documented by Englerth in Ref. 14, as well as computationally, documented by Schneider in Ref. 15. Conduction errors are important to the design of total temperature thermocouple probes for the use in jet engines because the mount by which the thermocouple is secured is generally actively cooled due to material temperature limits [14]. This can lead to large temperature gradients between the junction and the base of the thermocouple which will cause significant heat transfer that will reduce the temperature that the thermocouple indicates.

In Ref. 14, the effects of conduction errors were investigated for the total temperature probe seen in Figure 9. This probe was a straight tube, vented, shielded design and used an ungrounded, Inconel sheathed, Type K (Chromel-Alumel) thermocouple with Magnesium Oxide potting. The vented shield was made of Stainless Steel with four 0.0135 inch vent holes located about 0.2 inches from the leading edge.

The experimental hot jet facility used can be seen in Figure 10A.The probe was mounted in an airfoil strut that was actively cooled with either water or air to produce the desired conduction error effect. The strut was also coated with a thermal barrier coating (TBC) of Zirconia. The strut was secured to the nozzle using the brace seen in Figure 10B. The study focused on the recovery of the probe which is a performance metric that is defined as the fraction of the total temperature that the thermocouple indicates, as shown in Equation 6. To quantify the intensity of conduction that existed in the study, a 
dimensionless conduction driver was defined in Equation 7, where $T_{b}$ is the base temperature where the probe joins the strut.

$$
\begin{aligned}
& R=\frac{T_{j}}{T_{t}} \\
& \theta=\frac{\left(T_{t}-T_{b}\right)}{T_{t}}
\end{aligned}
$$

Physically, the conduction driver is the driving temperature difference within the thermocouple, normalized by the total temperature. Therefore, the driver is a number between zero and one, where a large conduction driver, close to unity, will indicate that the base temperature is much lower than the total temperature and therefore a large amount of heat loss due to conduction will exist. On the contrary, a low conduction driver, close to zero, will indicate that the base temperature is close to the total temperature of the flow and will result in a low value of conduction heat transfer. It was found that conduction error can be characterized by non-dimensional numbers, specifically the Reynolds number, $R e=\frac{\rho U D}{\mu}$; the Biot number, $B i=\frac{h d}{k_{S}}$; and the Nusselt number, $N u=\frac{h d}{k_{f}}$ [14]. Experiments were conducted at Mach numbers between 0.1 and 0.8 and total temperatures of $550^{\circ} \mathrm{F}$ and $850^{\circ} \mathrm{F}$. An important finding was that for a constant conduction driver, the recovery was independent of total temperature, that is, the Reynolds number and conduction driver could be used to completely characterize the recovery of the probe. This result can be seen in Figure 11 where the data was created using a thermal resistance model. For specific details, the reader is referred to Ref. 14. It should be mentioned, however, that these results were from a low-order model that included several simplifications. For example, the heat transfer via conduction was modeled simply by a Nusselt number correlation from Ref. 9 which was actually derived for a bare-wire thermocouple bead. To use this correlation, the Reynolds number was scaled to account for the lower velocity inside the shield and thus over the sensing element. Also, the results are plotted as a function of the Reynolds number based on the inner diameter of the shield; however, it could be argued that the diameter of the thermocouple sheath would be more physical and would relate better to the Nusselt number correlation. Nevertheless, despite these simplifications, the figure does show the theoretical collapse of the data on the two main non-dimensional parameters.

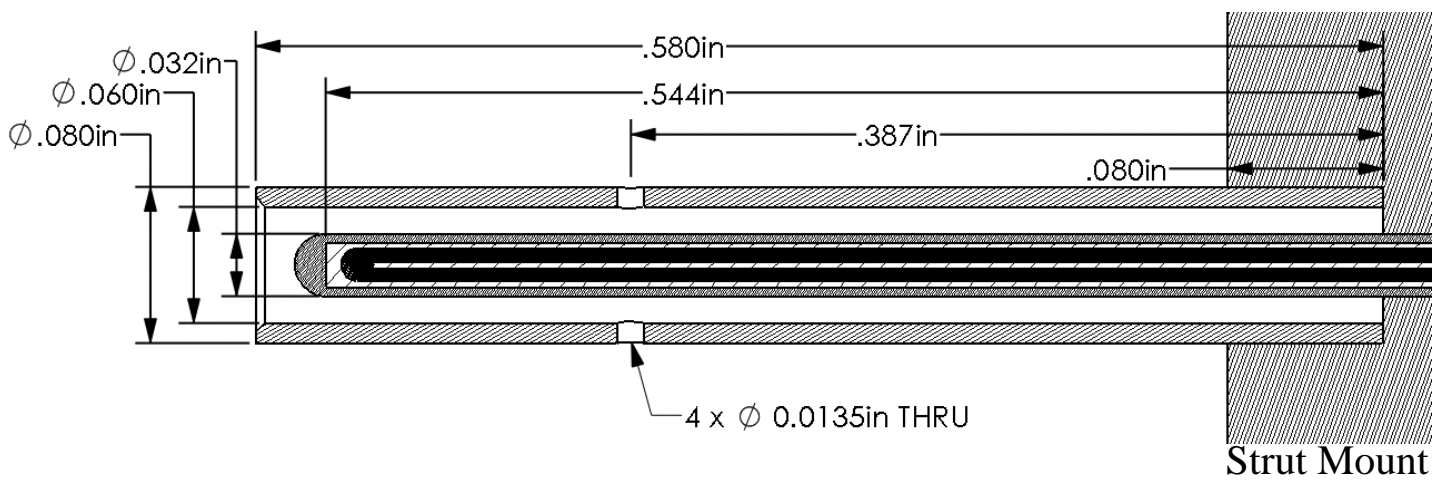

Figure 9: Shielded Probe used in Conduction Error Experiments at Virginia Tech 


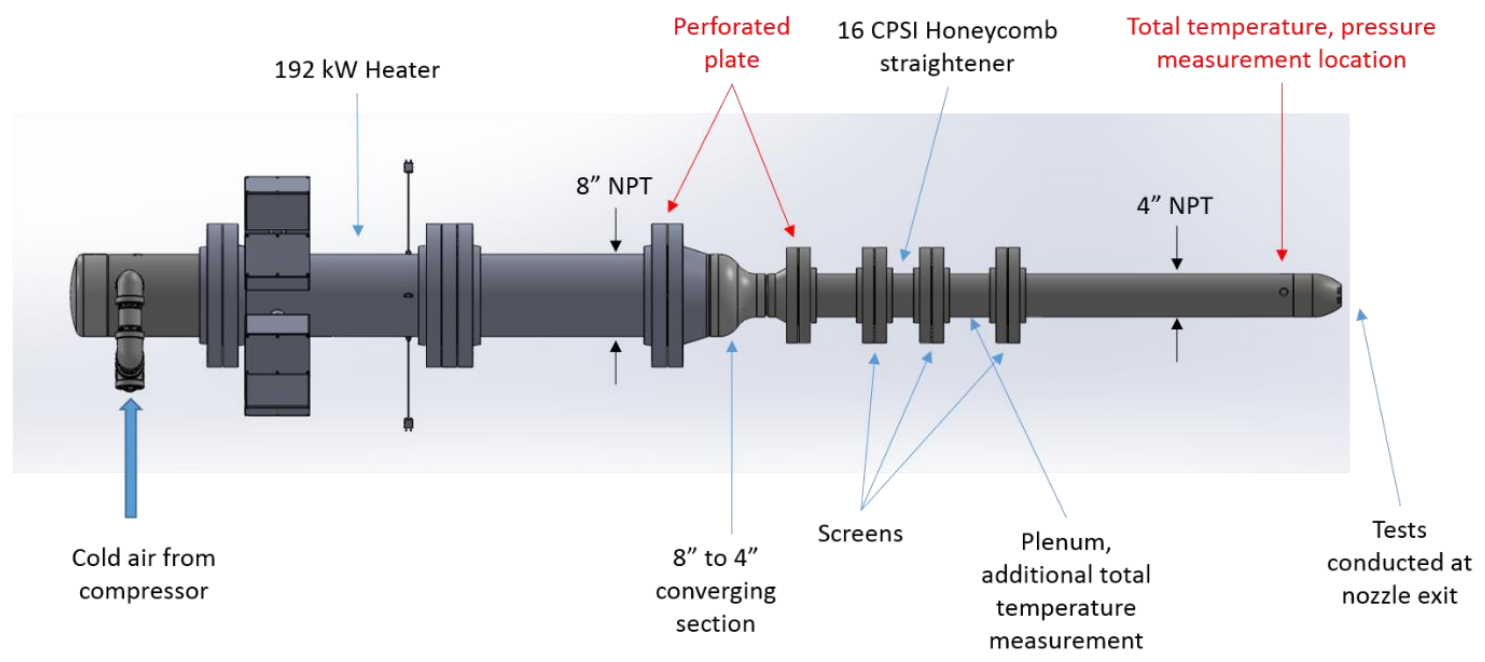

A) Overview of Hot Jet Facility

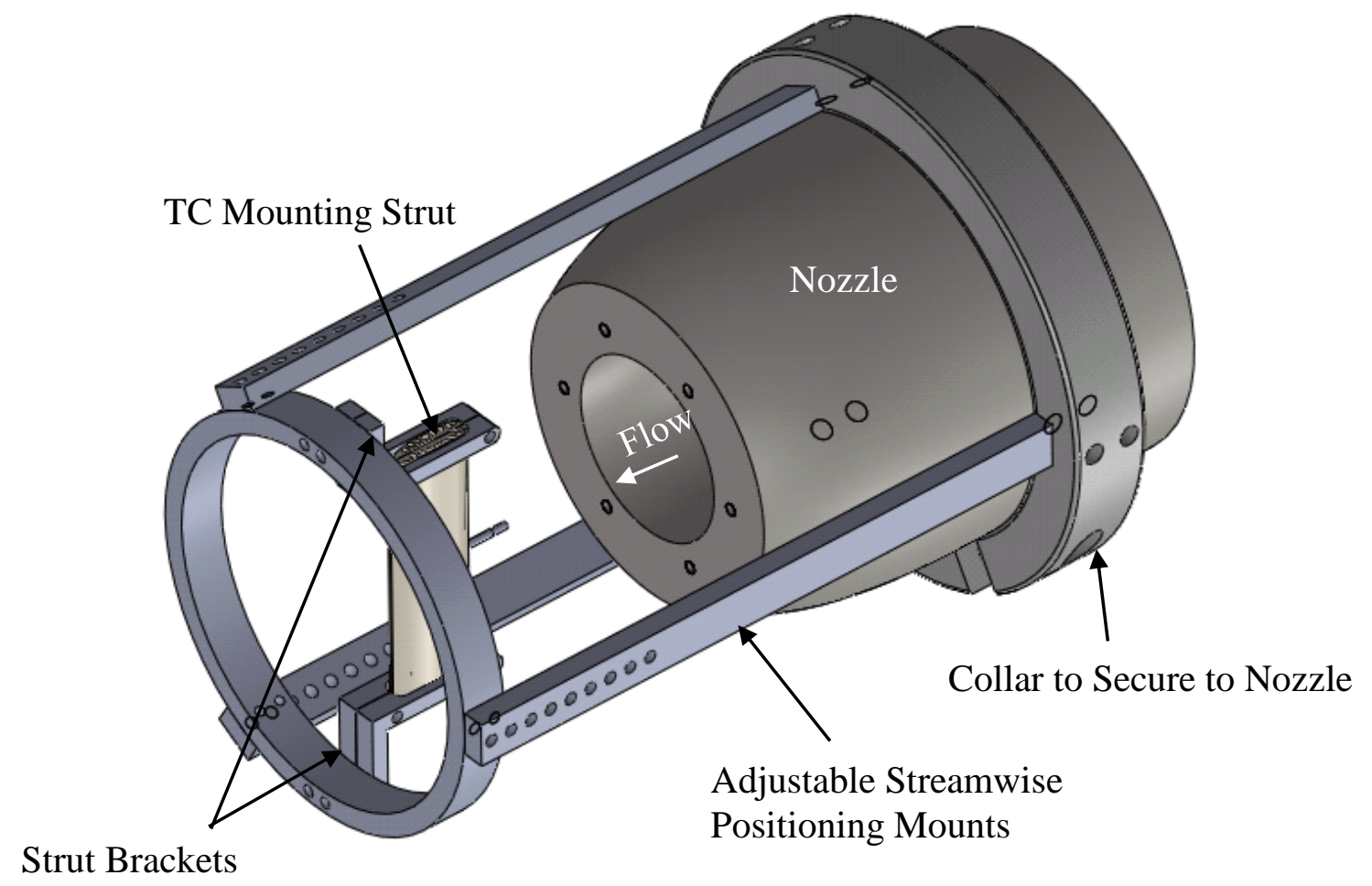

B) Airfoil Strut and Nozzle Mounted Brace

Figure 10: Experimental Setup of Virginia Tech Hot Jet Facility 


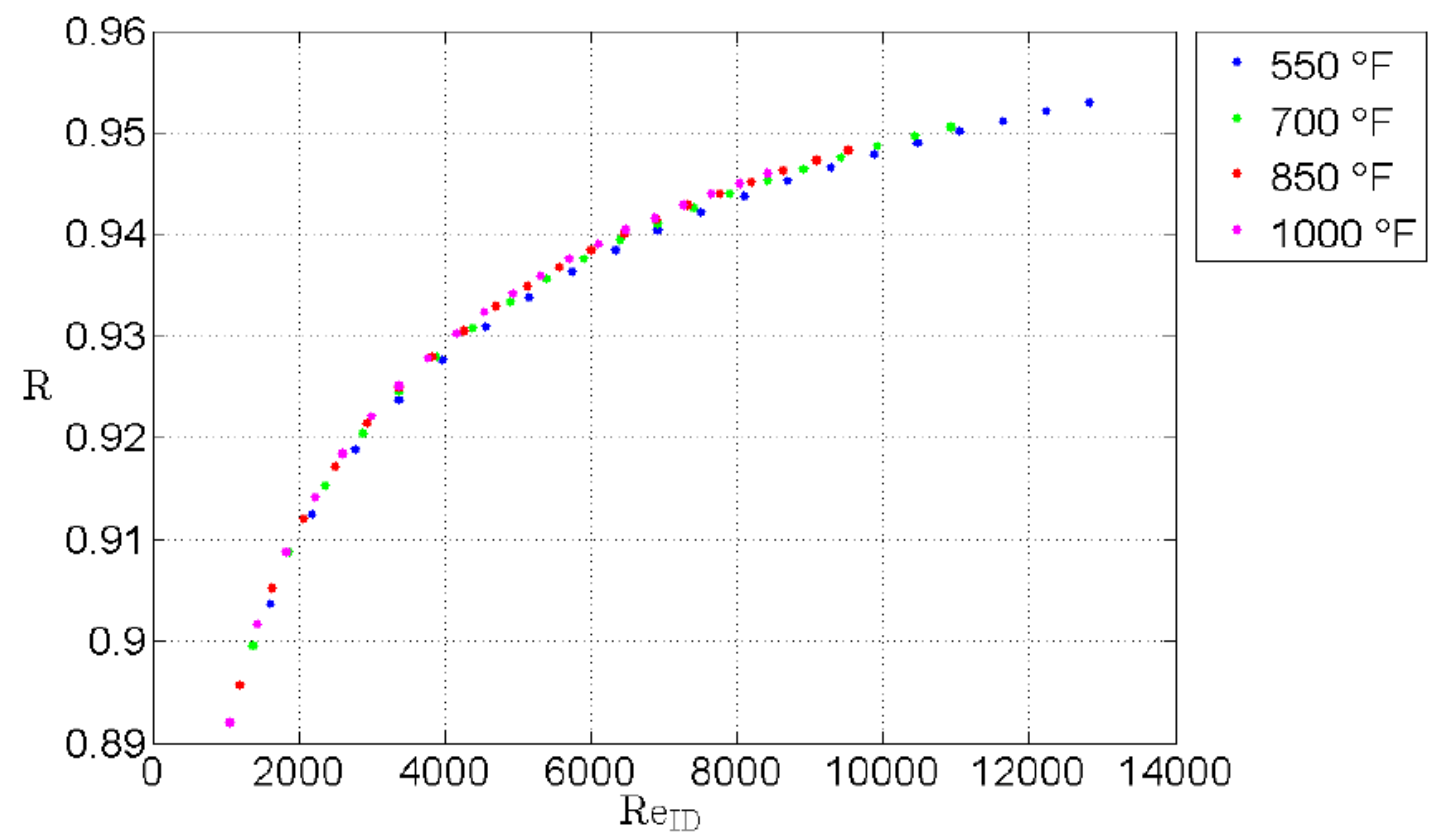

Figure 11: Recovery Results from Thermal Resistance Model from Ref. 14

In parallel, the ability to use computational models to simulate and predict the probe performance was studied by Schneider in Ref. 15. Schneider used the aerodynamic recovery correction work of Glawe et al. presented in the previous section as a validation case for low temperature simulations. From this he showed the ability to use multiphysics computer simulations to accurately model the flow and heat transfer processes that occur over a total temperature thermocouple. Good agreement was found between the simulations and the validation case so the methods were extended to the experimental results obtained by Englerth. For details, the reader is referred to Ref. 15.

The work of Schneider [15] has shown the usefulness of computation simulations in the design and analysis of total temperature thermocouple sensors. However, his work neglected the effect of radiation because of the moderate temperatures tested experimentally. Therefore, the current study seeks to extend those results and the capability of computational simulations to include the effects of radiation and to determine if radiation affected the probe's performance in the experimental work.

\section{Computational Methods}

The current study builds on the advanced multi-physics computational simulations developed in Ref. 15. A commercial Computational Fluid Dynamics (CFD) software was utilized. In particular, ANSYS FLUENT was chosen because of its broad capabilities and ready availability in the design community. Also, the program has the capability of performing Conjugate Heat Transfer (CHT) calculations simultaneously, which is the main focus of this study. A brief review of the major aspects of CFD and CHT simulations will be presented next. 


\subsection{Governing Equations for Computational Fluid Dynamics}

The basic principles used in fluid mechanics are the conservation of mass, momentum and energy. These equations are generally referred to as the Navier-Stokes equations for viscous fluid flows and can be derived in either a differential or integral form based on either an infinitesimal or finite control volume [16]. Here, the differential form will be presented in conservation form. A detailed discussion and derivation of these equations can be found in Refs. 16-18 among others.

\subsubsection{The Navier-Stokes Equations}

The continuity equation which expresses the principle of conservation of mass is given as

$$
\frac{\partial \rho}{\partial t}+\nabla \cdot(\rho \vec{V})=0
$$

where $\vec{V}=u \hat{\imath}+v \hat{\jmath}+w \hat{k}$ is the velocity vector shown in Cartesian coordinates. The momentum equation is a vector relation. The three components again shown in Cartesian coordinates are given as

$$
\begin{aligned}
& \frac{\partial(\rho u)}{\partial t}+\nabla \cdot(\rho u \vec{V})=-\frac{d P}{d x}+\frac{\partial \tau_{x x}}{\partial x}+\frac{\partial \tau_{y x}}{\partial y}+\frac{\partial \tau_{z x}}{\partial z}+\rho f_{x} \\
& \frac{\partial(\rho v)}{\partial t}+\nabla \cdot(\rho v \vec{V})=-\frac{d P}{d y}+\frac{\partial \tau_{x y}}{\partial x}+\frac{\partial \tau_{y y}}{\partial y}+\frac{\partial \tau_{z y}}{\partial z}+\rho f_{y} \\
& \frac{\partial(\rho w)}{\partial t}+\nabla \cdot(\rho w \vec{V})=-\frac{d P}{d z}+\frac{\partial \tau_{x z}}{\partial x}+\frac{\partial \tau_{y z}}{\partial y}+\frac{\partial \tau_{z z}}{\partial z}+\rho f_{z}
\end{aligned}
$$

where $\tau_{i j}$ is the flow stress tensor and $\vec{f}=f_{x} \hat{\imath}+f_{y} \hat{\jmath}+f_{z} \hat{k}$ is body force vector if body forces like gravity are present. Finally, the conservation of energy expressed in terms of internal energy is given in Equation 12. It should be noted that for turbulent flows, the thermal conductivity of the fluid $k_{f}$ is replaced by an effective thermal conductivity, $k_{\text {eff, }}$, which is the sum of the laminar and eddy thermal conductivities; the latter determined by an appropriate turbulence model.

$$
\begin{gathered}
\frac{\partial}{\partial t}\left[\rho\left(e+\frac{V^{2}}{2}\right)\right]+\nabla \cdot\left[\rho \vec{V}\left(e+\frac{V^{2}}{2}\right)\right]=\rho \dot{q}+\frac{\partial}{\partial x}\left(k_{f} \frac{\partial T}{\partial x}\right)+\frac{\partial}{\partial y}\left(k_{f} \frac{\partial T}{\partial y}\right)+\frac{\partial}{\partial z}\left(k_{f} \frac{\partial T}{\partial z}\right)- \\
\frac{\partial(P u)}{\partial x}-\frac{\partial(P v)}{\partial y}-\frac{\partial(P w)}{\partial z}+\frac{\partial\left(\tau_{x x} u\right)}{\partial x}+\frac{\partial\left(\tau_{y x} u\right)}{\partial y}+\frac{\partial\left(\tau_{z x} u\right)}{\partial z}+\frac{\partial\left(\tau_{y x} v\right)}{\partial x}+\frac{\partial\left(\tau_{y y} v\right)}{\partial y}+\frac{\partial\left(\tau_{z y} v\right)}{\partial z}+ \\
\frac{\partial\left(\tau_{x z} w\right)}{\partial x}+\frac{\partial\left(\tau_{y z} w\right)}{\partial y}+\frac{\partial\left(\tau_{z z} w\right)}{\partial z}+\rho \vec{f} \cdot \vec{V}
\end{gathered}
$$

These equations form a closed set when an equation of state such as the ideal gas law is included and when the shear stress terms are properly modeled. A brief discussion of turbulence models will be given next.

\subsubsection{The Reynolds-Averaged Navier-Stokes Equations for Turbulent Flows}

Turbulent flows by nature are inherently three dimensional and unsteady [17]. This makes computational simulations much more difficult than comparable laminar 
simulations. There are generally three ways to apply the Navier-Stokes equations to turbulent flows: using a Direct Numeric Simulation (DNS) approach, using a Large Eddy Simulation (LES) formulation, or using the Reynolds-Averaged Navier-Stokes (RANS) approach. Direct Numerical Simulation attempts to model all scales of turbulence including spatial and temporal fluctuations directly. However this becomes essentially impossible for any practical application of interest because the grid size and time step must be smaller than the smallest turbulent length and time scale given by the Kolmogrov scales $[17,18]$. A comprehensive review of the progress of DNS can be found in Ref. 19. Recently, Large Eddy Simulations (LES) has been developed to attempt to bridge the gap between DNS and RANS simulations by fully resolving some scales while modeling others. However, the run times are still prohibitive for routine applications.

In practical engineering applications, the instantaneous fluctuations of quantities are not necessary; a mean or time averaged quantity is more useful. Therefore, a common approach to solving turbulent flows is to use the time average of the Navier-Stokes equations. To do this, the velocity components are broken into a mean value and a fluctuating value. For example, the velocity component in the $\mathrm{x}$-direction of a Cartesian coordinate system will be written as $u(x, y, z, t)=U(x, y, z)+u^{\prime}(x, y, z, t)$, where $U$ is the mean value of the $\mathrm{x}$-velocity at a point $\mathrm{x}, \mathrm{y}, \mathrm{z}$ and $u^{\prime}$ is the instantaneous fluctuation of $\mathrm{x}$-velocity at this point. By replacing the variables in the Navier-Stokes equations, and taking the time average, the equations can be used to solve for mean flow quantities. A thorough derivation of these equations can be found throughout the literature, e.g. Ref. 17. An important result can be seen by inspecting the time-averaged momentum equation, expressed in index form below. It is important to note the introduction of a new term, $-\rho \overline{u_{\imath}{ }^{\prime} u_{\jmath}{ }^{\prime}}$. This term is known as the Reynolds stress and must be modeled to close the system of equations [17]. There are many ways to model this turbulent shear stress term, and a brief description of the most popular models will be given in the next section.

$$
\frac{\partial}{\partial t}\left(\rho u_{i}\right)+\frac{\partial}{\partial x_{j}}\left(\rho u_{i} u_{j}\right)=-\frac{\partial P}{\partial x_{i}}+\frac{\partial}{\partial x_{j}}\left[\mu\left(\frac{\partial u_{i}}{\partial x_{j}}+\frac{\partial u_{j}}{\partial x_{i}}-\frac{2}{3} \delta_{i j} \frac{\partial u_{l}}{\partial x_{l}}\right)\right]+\frac{\partial}{\partial x_{j}}\left(-\rho \overline{u_{l}{ }^{\prime} u_{\jmath}^{\prime}}\right)
$$

\subsection{Turbulence Models}

Turbulence modeling is a vast subject in itself and many models have been developed. These range from simple algebraic models or zero-equation models, to one-equation, two-equation, and other multi-equation differential models. Only a brief discussion will be given here but the reader is directed to the numerous works in the literature on the subject for more in depth discussions of specific models.

The attempt to develop turbulence models have generally followed two approaches: the eddy viscosity approached developed by Boussinesq and shown in Equation (14) and the mixing length approach developed by Prandtl shown in Equation (15) [16, 17].

$$
\tau_{t}=-\rho \overline{u^{\prime} v^{\prime}}=\rho v_{T} \frac{\partial U}{\partial y}
$$




$$
\tau_{t}=-\rho \overline{u^{\prime} v^{\prime}}=\rho l_{m}^{2}\left|\frac{\partial U}{\partial y}\right| \frac{\partial U}{\partial y}
$$

In these equations, $\mu_{T}$ is the turbulent eddy viscosity and $l_{m}$ is the mixing length. The eddy viscosity resembles the laminar viscosity but depends not only on the fluid but also the flow; that is, it is not a fluid property [17]. The mixing length represents an "effective interaction distance" between eddies within the flow [17]. The above equations are formulated for two-dimensional flows. However, attempts to extend them to three dimensional flows have been made by Smagorinsky and Baldwin and Lomax [18].

\subsubsection{Zero-Equation Models}

The simplest approach to modeling turbulence is to use a zero-equation model, that is, a model that is algebraic with no differential equations. A variety of models have been developed. Popular among these are the Cebeci and Smith (CS) Model and the BaldwinLomax Model. It has been shown that these models are particularly accurate for attached boundary layer flows [18]. The reader is referred to Refs. 16 and 20 for more specific information regarding these models.

\subsubsection{One-Equation Models}

Perhaps the most well-known one equation model developed is the Spallart-Almaras Model. This model includes one differential transport equation that is semi-empirical to define the eddy viscosity $[18,20]$. This formulation was created specifically for external aerodynamics and thus produces the best results when used for such applications [18]. For specific details, the reader is referred to Refs. 18 and 21.

\subsubsection{Two-Equation Models}

There two well-known two-equation models, the $k-\varepsilon$ model and the $k$ - $\omega$ model. The $k-\varepsilon$ model was developed by Jones and Luander [22] and defines the eddy viscosity as $v_{T}=$ $c_{m} k^{2} / \varepsilon$, where $c_{m}$ is a constant, and $k$ and $\varepsilon$ are the turbulent kinetic energy and rate of dissipation, respectively and are found from two separate transport equations [18]. This formulation performs well for free shear flows but must be adjusted for use in wall bounded flows [18].

The $k-\omega$ model due to Wilcox is similar to the $k-\varepsilon$ model but provides better accuracy in the presence of walls [18]. In this model, the turbulence frequency is defined as $\omega=\varepsilon / k$ and the eddy viscosity is calculated in the same way as in the $k-\varepsilon$ model. Again, these two quantities are found with separate transport equations [20]. A recent development of the $k-\omega$ model is the $k-\omega$ Shear Stress Transport (SST) model developed by Menter. This model combines aspects of the $k-\varepsilon$ to be used in the free stream flow away from any walls and uses a blending function that transitions to a $k-\omega$ formulation at the wall [18]. The $k$ $\omega$ SST model will be used throughout this work. 
The SST formulation transforms the $k-\varepsilon$ model equations into a $k$ - $\omega$ formulation and multiplies the equations of both the $k-\omega$ model and the $k-\varepsilon$ model by a blending function. The two equations for $\mathrm{k}$ and $\omega$ can be seen below, where $\Gamma_{k}$ and $\Gamma_{\omega}$ are the effective diffusivity of $k$ and $\omega, \widetilde{G_{k}}$ represents the generation of $k$ from mean velocity gradients, $G_{\omega}$ represents the generation of $\omega, Y_{k}$ and $Y_{\omega}$ represent the dissipation of $k$ and $\omega, S_{k}$ and $S_{\omega}$ are source terms, and $D_{\omega}$ represents a cross diffusion term. For specific information how these quantities are calculated in FLUENT, the reader is directed to Ref. 23.

$$
\begin{aligned}
& \frac{\partial}{\partial t}(\rho k)+\frac{\partial}{\partial x_{i}}\left(\rho k u_{i}\right)=\frac{\partial}{\partial x_{j}}\left(\Gamma_{k} \frac{\partial k}{\partial x_{j}}\right)+\widetilde{G_{k}}-Y_{k}+S_{k} \\
& \frac{\partial}{\partial t}(\rho \omega)+\frac{\partial}{\partial x_{j}}\left(\rho \omega u_{j}\right)=\frac{\partial}{\partial x_{j}}\left(\Gamma_{\omega} \frac{\partial \omega}{\partial x_{j}}\right)+G_{\omega}-Y_{\omega}+D_{\omega}+S_{\omega}
\end{aligned}
$$

The eddy viscosity is then calculated as in Equation 18, where $a^{*}$ is a coefficient that damps the turbulent viscosity, $F_{2}$ is a blending function, $S$ is the magnitude of the strain rate, and $a_{1}$ is a model constant.

$$
\mu_{t}=\frac{\rho k}{\omega} \frac{1}{\max \left[\frac{1}{a^{*}}, \frac{S F_{2}}{a_{1} \omega}\right]}
$$

From this eddy viscosity, the effective conductivity (the sum of the laminar and eddy conductivities) can be calculated. For example, in the areas that the $k$ - $\varepsilon$ model is employed, the effective conductivity is given as in Equation 19, where $\operatorname{Pr}_{T}$ is the turbulent Prandtl number with a default value of 0.85 .

$$
k_{e f f}=k_{f}+\frac{C_{p} \mu_{t}}{P r_{T}}
$$

\subsubsection{Multi-Equation Models}

There are many other turbulence models that utilize more than two equations. For example, the $\overline{v^{2}}-f$ model developed by Durbin [24] is a four equation model developed from the $k-\varepsilon$ by adding two additional equations. The reader can consult the literature for further details, specifically Refs. 17 and 24 for example.

\subsection{Conjugate Heat Transfer}

In addition to calculating fluid flows, many CFD codes have incorporated multi-physics models, most common of which is the capability to calculate conjugate heat transfer. This allows for the calculation of heat transfer by conduction and sometimes radiation as well as convection due to the bulk fluid flow. To solve a flow problem that includes heat transfer, the appropriate heat transfer models are included in the energy equation. For example, the governing model of heat conduction is Fourier's Law given as $q^{\prime \prime}=-k \nabla T$, where $k$ is the thermal conductivity of the medium. This corresponds to the second, third, and fourth terms of the energy equation given in Equation 12. 
The fundamental physical law for convection is also Fourier's Law; however, it is traditional to write convective heat transfer using Newton's Law of Cooling given as $q=$ $h A_{c}\left(T_{\infty}-T_{s}\right)$ where $h$ is the convective heat transfer coefficient, $A_{C}$ is the surface area, and $T_{\infty}$ and $T_{S}$ are the freestream flow temperature and the surface temperature, respectively. It is important to note that the convective heat transfer coefficient is not a fluid property but depends on the nature of the flow.

To calculate the heat transfer in turbulent flows, a simple approach is made by modifying the laminar formulation by adding an eddy conductivity, much like the eddy viscosity is done in the momentum equation [25]. The result for the heat flux in the $n$ direction is $\dot{q}^{\prime \prime}=-\rho C_{p}\left(\frac{v}{P r}+\frac{v_{T}}{P r_{T}}\right) \frac{\partial T}{\partial n}$, where $v_{T}$ is the eddy viscosity and $\operatorname{Pr}_{T}$ is the turbulent Prandtl number defined as $P r_{T}=\frac{v_{T}}{k_{T} / \rho C_{p}}$ where $k_{T}$ is the turbulent eddy conductivity, $\rho$ is the average density and $C_{p}$ is the specific heat at constant pressure.

Finally, the radiation heat transfer between a surface and the surroundings can be given as $q=\varepsilon_{w} \sigma A_{R}\left(T_{s}{ }^{4}-T_{\text {surr }}{ }^{4}\right)$. This equation shows why radiation becomes so important at high temperatures: due to the effect of the fourth power. In practice, computational codes solve the Radiative Transport Equation (RTE), seen below to include scattering and absorption effects within the gaseous mediums as well as exchange between multiple surfaces. In particular, the Discrete Ordinance (DO) Model will be used in this work. This model solves the below RTE for a range of direction vectors, $\vec{s}$, based on a user defined discretization of the solid angle [23]. This model has the ability to include diffuse and/or specular radiation, opaque or transparent surfaces, as well as the effects of participating media (absorption and scattering).

$$
\nabla \cdot(I(\vec{r}, \vec{s}) \vec{s})+\left(a+\sigma_{s}\right) I(\vec{r}, \vec{s})=a n^{2} \frac{\sigma T^{4}}{\pi}+\frac{\sigma_{s}}{4 \pi} \int_{0}^{4 \pi} I(\vec{r}, \vec{s}) \Phi\left(\vec{s} \cdot \vec{s}^{\prime}\right) d \Omega^{\prime}
$$

For an opaque wall, incident radiation can be absorbed or reflected while the wall can also emit radiation. The DO model calculates these quantities as shown in Table 2 where $n$ is the refractive index, $\varepsilon_{w}$ is the emissivity of the surface, $f_{d}$ is the diffuse fraction and $q_{\text {incident }}$ is the incident radiation at the surface. For a surface that reflects radiation diffusely, the diffuse fraction is 1 , and for a specular surface reflection, the diffuse fraction is 0 . The reader is reminded that the below equations make use of Kirchhoff's Law which states that for heat transfer in a blackbody cavity the emissivity of a surface is equal to its absorptivity $\left(\alpha_{w}\right)$. Therefore, it follows for an opaque surface, the reflectivity can be defined as $\rho_{w}=1-\alpha_{w}=1-\varepsilon_{w}$. 
Table 2: Calculation of Radiation Quantities in DO Model

\begin{tabular}{|c|c|}
\hline Quantity & Equation \\
\hline \hline Emission from Wall & $n^{2} \varepsilon_{w} \sigma T_{w}{ }^{4}$ \\
\hline Diffusely Reflected Radiation & $f_{d}\left(1-\varepsilon_{w}\right) q_{\text {incident }}$ \\
\hline Specular Reflected Radiation & $\left(1-f_{d}\right)\left(1-\varepsilon_{w}\right) q_{\text {incident }}$ \\
\hline Absorbed Radiation & $\varepsilon_{w} q_{\text {incident }}$ \\
\hline
\end{tabular}

\subsection{Meshing}

The physical and model equations given must be solved at discrete points in the domain. Therefore, a computational grid must be developed; this is called a mesh. There are two common types of meshes that are used, structured and unstructured. Structured meshes can allow for quicker computations [17]. However, for complex geometries, creating a structured mesh becomes difficult, although there are procedures that allow for their development. An alternate approach is to use an unstructured mesh where the elements are not ordered.

An important component of the mesh for fluid flows is the inflation layer. This is a thin region of elements near the surface of a body which is used to resolve the large gradients near the wall due to the boundary layer. It is generally desired to have the first layer of the inflation layer at a height corresponding to $\mathrm{y}^{+} \sim 1$, where the $\mathrm{y}^{+}$is a non-dimensional height defined as $y^{+}=\frac{y u_{*}}{v}$, where $y$ is the distance perpendicular from the surface, $u_{*}=$ $\sqrt{\tau_{w} / \rho}$ is the friction velocity, and $v$ is the kinematic viscosity [17]. The $y^{+}$parameter relates the element height off the surface to the small scale turbulent processes at the wall and can be used as an indication of whether the region directly near the wall is resolved well enough.

\section{Computational Setup}

Computations were undertaken for three thermocouple sensors. The first two sensors were the shielded and unshielded probes from the work of Glawe et al. as described in Section 2.1 and they were used as validation for the computational methods. The third probe modeled was the sensor developed at Virginia Tech to investigate conduction errors as discussed in Section 2.2. In each case, the same procedure was followed. First, a computer-aided-design (CAD) model of the geometry and fluid domain of interest was developed in SolidWorks. This model was then spatially discretized using the meshing ability of ANSYS Workbench. After creating the mesh, the flow field boundary conditions were applied and the simulation was conducted in ANSYS FLUENT. Finally, post processing was done in ANSYS CFD Post. In the following sections, this process will be outlined for each case starting first with the validation case, followed by the conduction error case. It should be noted that the validation case was performed prior to the conduction error case. However, because the general methods applied to both cases 
were identical, their development will be given simultaneously in the following sections. It will be shown that the validation case indeed confirmed the ability to use computational simulations to model and predict the performance of these thermocouple sensors.

\subsection{Validation Case from Ref. 10}

As previously mentioned, the shielded and unshielded thermocouple sensors from the work of Glawe, Simmons, and Stickney were chosen as a validation case. These probes were chosen because they were extensively documented in Ref. 10 and were designed to have negligible conduction errors and minimal aerodynamic errors at subsonic Mach numbers.

\subsubsection{Geometry and Meshing}

To conduct this validation, the physical geometries were replicated using a computeraided-design (CAD) program. The region modeled was to represent the fluid region immediately downstream of the four inch diameter nozzle as shown in Figure 12. To reduce the complexity of the simulation, a three-dimensional model representing a $45^{\circ}$ sector of the region was developed. This was done to reduce the computational expense that accompanies a full three-dimensional simulation and to take advantage of the assumed symmetry of the flow. The region was broken into $45^{\circ}$ sections because of the location of the vent holes on the shielded probe. It is important to note that because the symmetric model was created, the bare-wire thermocouple pair could not be modeled explicitly. Instead, the thermocouple pair was modeled as a single "rod" with the same cross sectional area as the two wires. This three-dimensional model was developed for the shielded probe only and is shown in Figure 13.

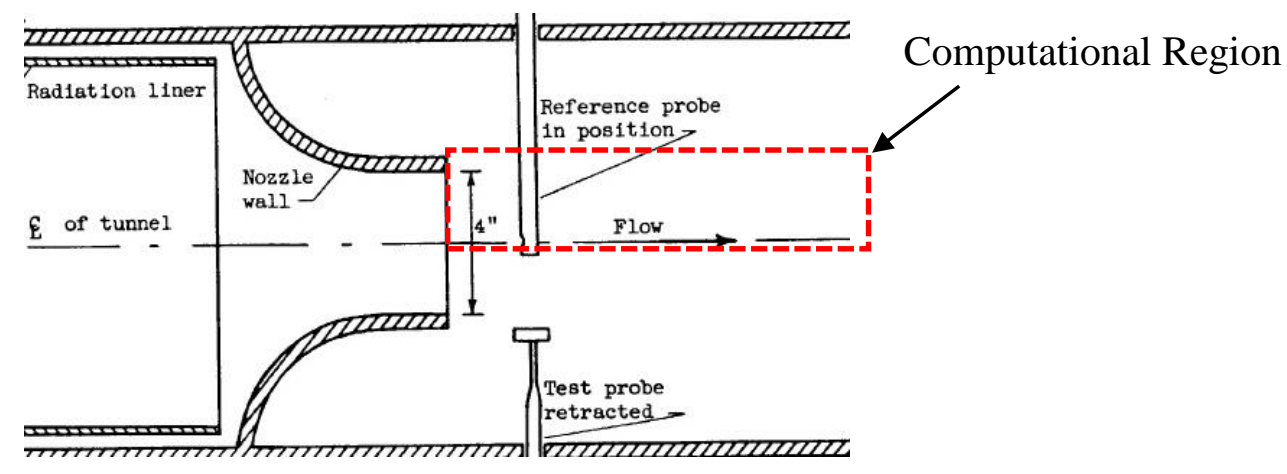

Figure 12: Computational Domain for Validation Cases 


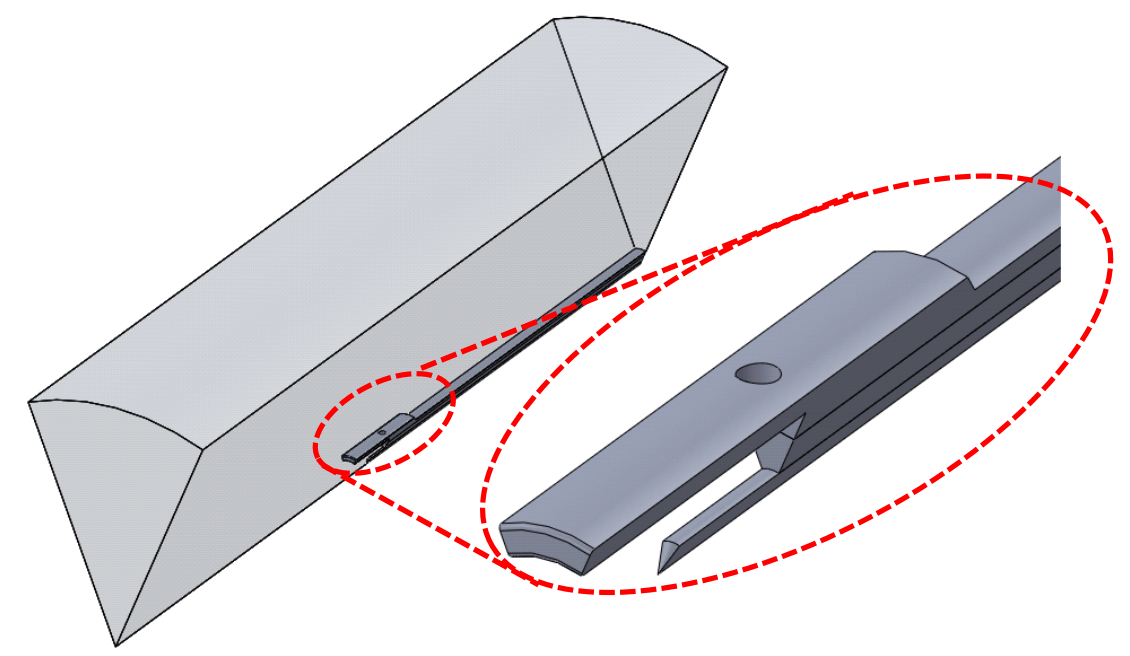

Figure 13: Three Dimensional Geometry for NACA Shielded Thermocouple Sensor

In addition to the three-dimensional symmetric model, two-dimensional axisymmetric models were created. This was done again to further reduce the computational time and to allow for more rapid parametric studies. Two-dimensional, axisymmetric models were created for both the shielded and unshielded NACA probes. Again, several modifications to the geometry had to be made. As was the case with the three-dimensional symmetric model, the bare-wire thermocouple pair had to be modeled as a single "rod". Also, for the shielded probe, the vent holes needed to be modeled as a single "slot" due to the axisymmetric definition. This slot was sized to allow the same mass flow rate as the circular vent holes. Further information can be found in Ref. 15. Axisymmetric models of the shielded and unshielded NACA sensors can be seen in Figure 14 below.

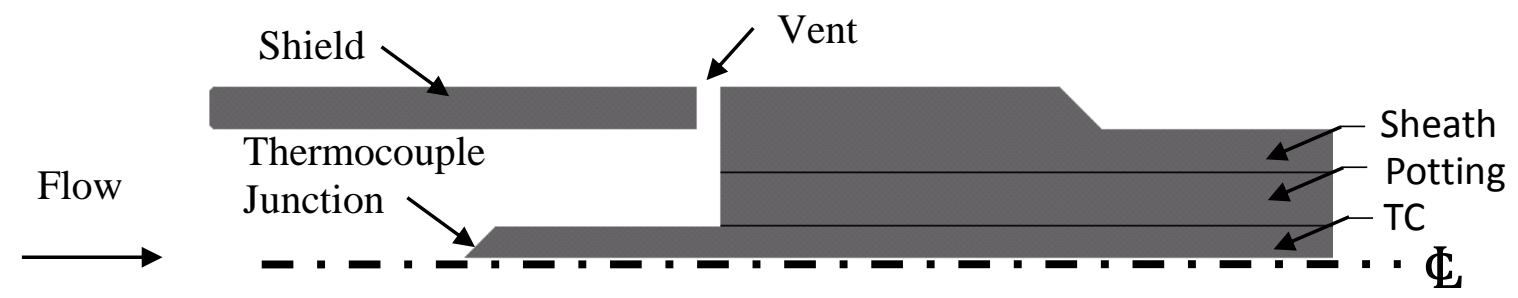

A) Shielded Probe

Thermocouple

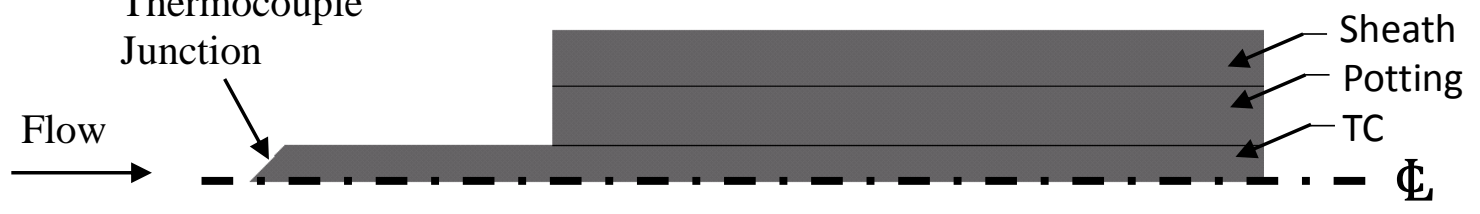

B) Unshielded Probe

Figure 14: Two-Dimensional, Axisymmetric Geometries for the NACA Sensors

After creating the CAD geometries, the computational domains had to be spatially discretized, or meshed. The three-dimensional model of the shielded probe was meshed using an unstructured grid developed in ANSYS Mechanical, the meshing program in 
ANSYS Workbench. An unstructured mesh was chosen because of complex curves and sweeps in the geometry, in particular in and around the shield [15]. The mesh was composed of tetrahedral elements with an inflation layer made of prisms to resolve the boundary layer that would develop on the surface of the solid components. The internal solid components were also meshed with an unstructured approach again using tetrahedral elements. Also, a manual region of refinement was created around the shield and exposed thermocouple to increase resolution in this region of interest. The final mesh had a total of 4,007,117 elements. This mesh can be seen in Figure 15.

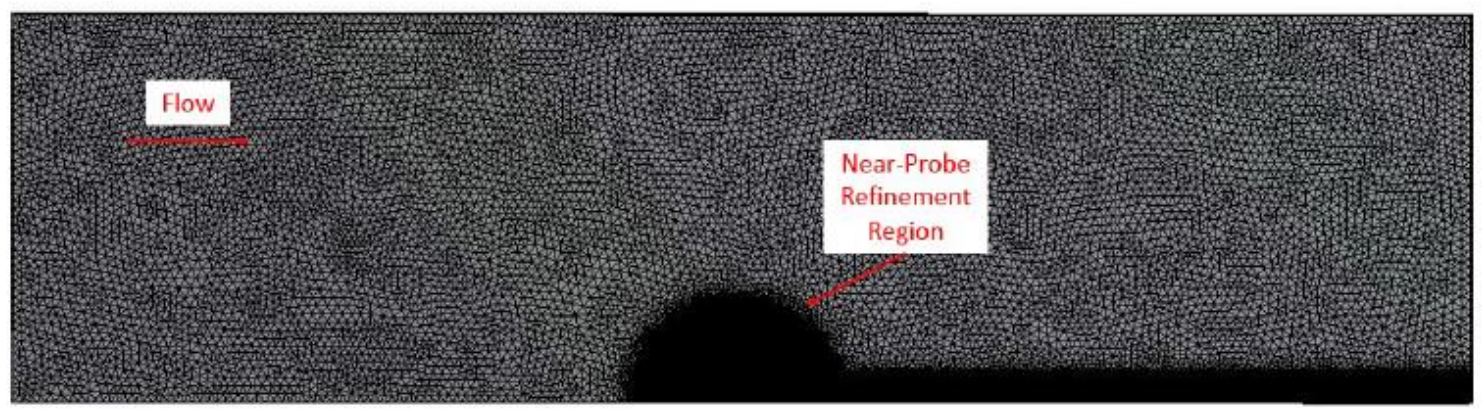

A) Overall Mesh

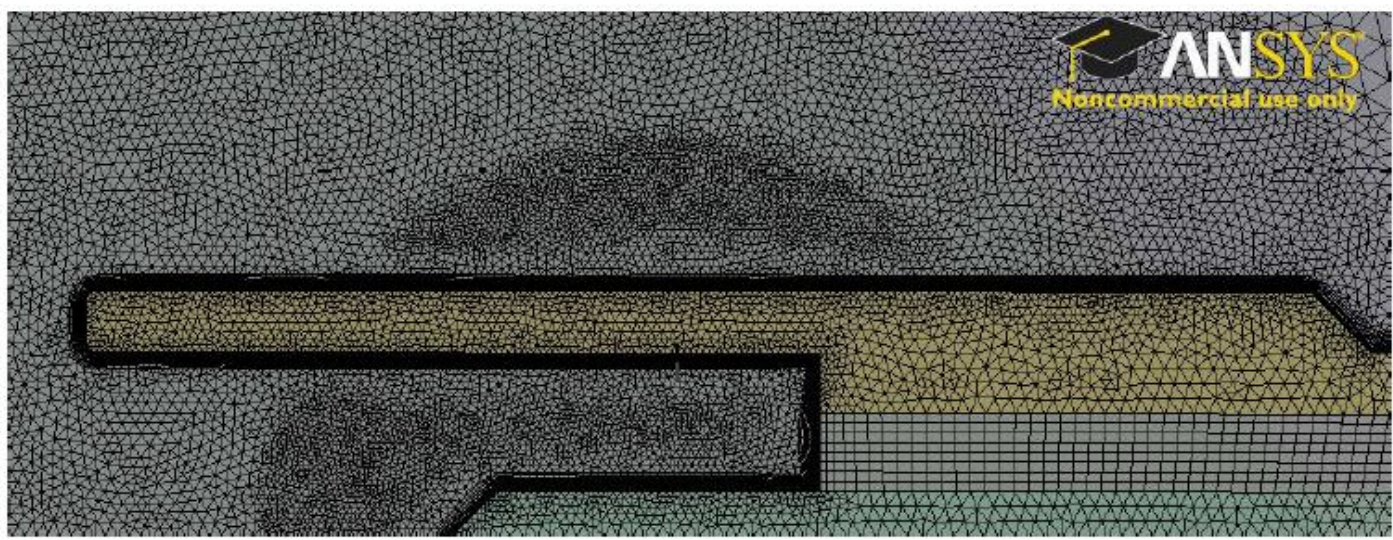

B) Detailed View of Probe Region

Figure 15: Unstructured Mesh for Three-Dimensional Model of Shielded Probe [15]

Similarly, the two-dimensional axisymmetric geometries were meshed with an unstructured grid. These meshes were composed of triangular elements in the fluid and quadrilateral elements in the inflation layer and in the solid components. Again, a refinement region around the thermocouple junction was created manually to ensure that this region was adequately resolved. The number of elements was much less in the axisymmetric meshes than in the three dimensional meshes. The total number of elements were 208,066 and 136,929 for the shielded and unshielded geometries, respectively. The mesh for the shielded probe is shown in Figure 16. 


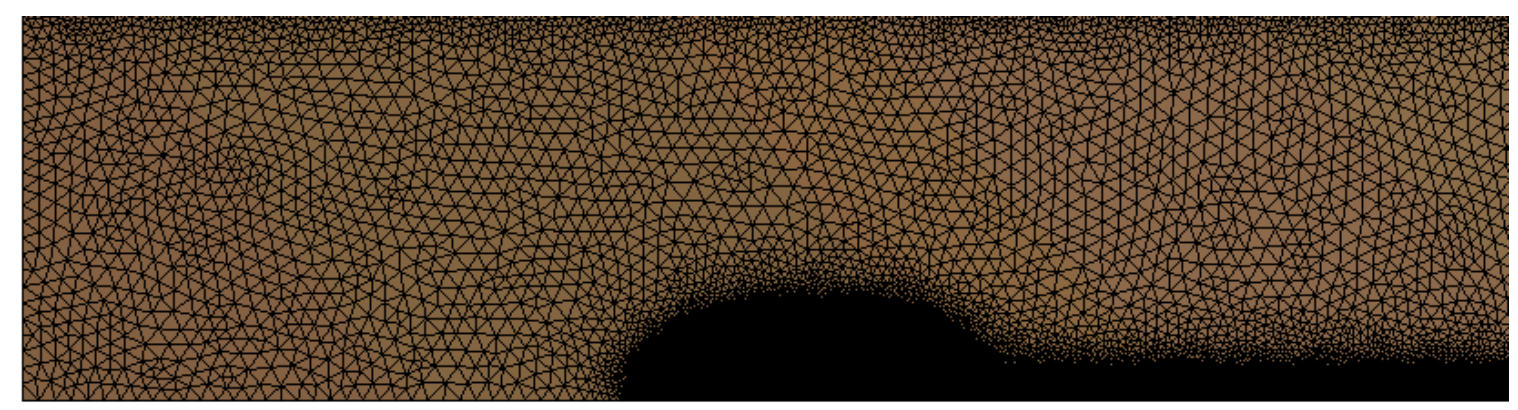

A) Overall Mesh

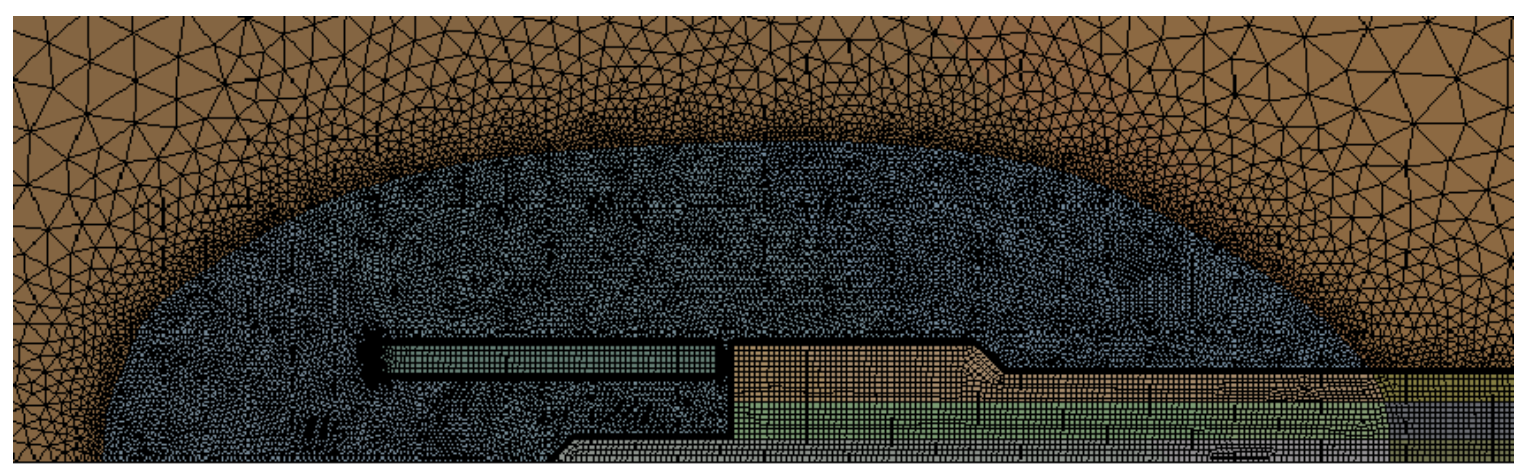

B) Detailed View of Probe Region

Figure 16: Unstructured Mesh for Two-Dimensional, Axisymmetric Model of Shielded Probe

The development of suitable meshes is not a trivial process as the quality of the mesh has great impacts on the accuracy of the computations. This is especially true at the wall-fluid interface where skin friction and heat transfer processes take place within the boundary layer. To ensure that the boundary layer was properly resolved, the $y^{+}$parameter was used. In general it is desired to have the first cell located within the laminar sublayer which corresponds to a $y^{+}<5$ [17]. The meshes used were within this range.

\subsubsection{Boundary Conditions and Flow Setup}

After creating the mesh, it was necessary to define the boundary conditions to represent the flow parameters in the experimental setup. In Ref. 15, a validation study based on the aerodynamic correction was conducted with simulations undertaken at a total temperature of $540 \mathrm{R}(300 \mathrm{~K})$ and a total pressure of 1atm with Mach numbers of 0.3, 0.6 and 0.9. The current study focused on extending these results to validate the computation of radiation heat transfer. These simulations were undertaken at total temperatures of $2500 \mathrm{R}$ $(1388.89 \mathrm{~K}), 2200 \mathrm{R}(1222.22 \mathrm{~K}), 1800 \mathrm{R}(1000 \mathrm{~K})$, and 1600R $(888.89 \mathrm{~K})$ all at a Mach number of 0.3 and a total pressure of $1 \mathrm{~atm}$ for comparison with the published data in Ref. 10. The boundary conditions were applied using Pressure Farfield conditions to specify the freestream conditions. In addition, for the two-dimensional axisymmetric simulations, the axis had to be defined and for the three-dimensional simulation the symmetry planes had to be defined as shown in Figure 17 and Figure 18. 


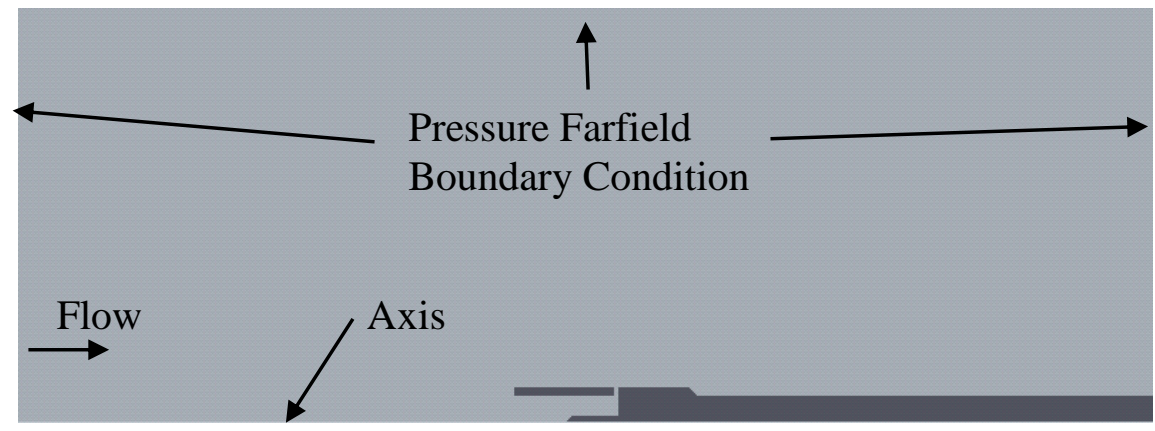

Figure 17: Boundary Conditions for Two-Dimensional, Axisymmetric Simulations

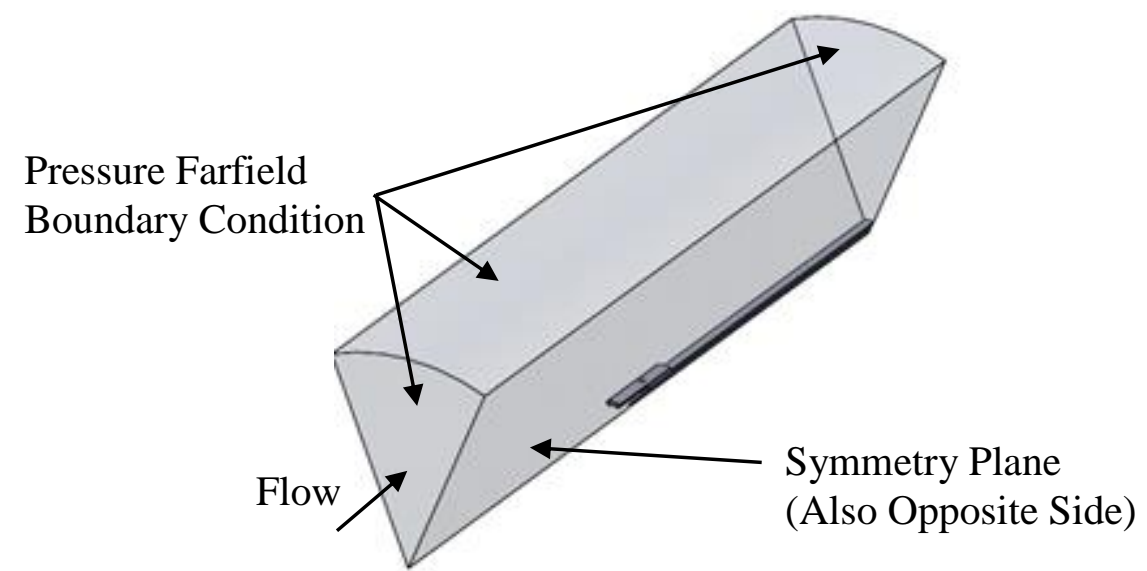

Figure 18: Boundary Conditions for Three-Dimensional Simulations

In addition to defining flow variables, the setup required specification of models for turbulence and heat transfer as was discussed previously. In all simulations, the $k-\omega \mathrm{SST}$ turbulence model was chosen for its wide applicability in a range of flows. Also, to compute the radiation heat transfer, the Discrete Ordinance (DO) Radiation model was chosen because of its generality.

It is important to note that an upstream radiation source was modeled using an emissivity of 0.1 and the total temperature to represent the radiation from a non-luminous flame in the combustor section. The surroundings were also defined as room temperature with an emissivity of 1.0 .

Finally, it was necessary to define the material properties of the solids and fluid modeled. The thermocouple was given "effective" properties of Chromel-Alumel because as previously mentioned, the single bare wires were not modeled, but a simple rod surrogate was employed instead. The thermal properties of the thermocouple materials were defined as presented in Table 3. 
Table 3: Thermo-physical Properties of Thermocouple Components used in Simulations of NACA Probes

\begin{tabular}{|c|c|c|c|c|}
\hline Component & Material & $\begin{array}{c}\text { Thermal } \\
\text { Conductivity }\end{array}$ & $\begin{array}{c}\text { Specific Heat at } \\
\text { Constant Pressure }\end{array}$ & Emissivity \\
\hline $\begin{array}{c}\text { Sheath \& } \\
\text { Shield }\end{array}$ & Inconel & $23.234 \mathrm{~W} / \mathrm{m}-\mathrm{K}$ & $722.964 \mathrm{~J} / \mathrm{kg}-\mathrm{K}$ & 0.3 \\
\hline Potting & $\begin{array}{c}\text { Magnesium } \\
\text { Oxide }\end{array}$ & $8.3164 \mathrm{~W} / \mathrm{m}-\mathrm{K}$ & $1273.24 \mathrm{~J} / \mathrm{kg}-\mathrm{K}$ & 0.16 \\
\hline TC & $\begin{array}{c}\text { Chromel- } \\
\text { Alumel }\end{array}$ & $24.45 \mathrm{~W} / \mathrm{m}-\mathrm{K}$ & $485.5 \mathrm{~J} / \mathrm{kg}-\mathrm{K}$ & 0.75 \\
\hline
\end{tabular}

The properties of the working fluid were also specified. Initially, the working fluid was assumed to be air as no documentation on the combustion products in the test apparatus was readily found. Air was modeled as an ideal gas and the thermal conductivity and specific heat at constant pressure were calculated by piecewise linear interpolation between temperature dependent data obtained from Ref. 26. Also, the viscosity was calculated using Sutherland's Law. The assumption of air as the working fluid was deemed acceptable because calculating the adiabatic flame temperature of the combustion products for several fuels to yield the temperatures studied required were on the order of $300 \%-400 \%$ excess air [12]. Therefore, the combustion products were assumed to be quite lean and their effects were assumed negligible.

Although the effects were assumed to be negligible, it was still uncertain whether the thermo-physical properties of the combustion products, particularly the specific heat capacity, thermal conductivity and the specific heat ratio, actually influenced the flow and heat transfer processes, especially at the higher total temperatures. Therefore, after further investigation, it was determined that the experimental tunnel was run on 72 Octane [27]. This knowledge allowed for a more accurate model to be developed that included the relevant thermodynamics. To do this, the equilibrium mass fractions of the combustion products was computed using the NASA Chemical Equilibrium with Applications (CEA) software [28]. As was expected, the analysis indicated that for all total temperatures tested, the combustion was lean. The mass fractions of the most abundant species in the combustion products that were modeled in the simulations are shown in Table 4. 
Table 4: Equilibrium Mass Fractions for Combustion of 72 Octane with Air used in Simulations [12]

\begin{tabular}{|c|c|c|c|c|c|}
\hline $\begin{array}{c}\text { Total } \\
\text { Temperature: }\end{array}$ & $\mathbf{1 6 0 0} \mathbf{R}$ & $\mathbf{1 8 0 0} \mathbf{R}$ & $\mathbf{2 0 0 0} \mathbf{R}$ & $\mathbf{2 2 0 0} \mathbf{R}$ & $\mathbf{2 5 0 0} \mathbf{R}$ \\
\hline $\begin{array}{c}\text { Equivalence } \\
\text { Ratio: }\end{array}$ & 0.22 & 0.27 & 0.315 & 0.365 & 0.445 \\
\hline \hline Argon & 0.012731 & 0.01269 & 0.012653 & 0.012612 & 0.012547 \\
\hline $\mathbf{C O 2}$ & 0.044649 & 0.05451 & 0.063331 & 0.073072 & 0.088526 \\
\hline $\mathbf{H 2 O}$ & 0.020342 & 0.024884 & 0.028946 & 0.033432 & 0.040544 \\
\hline $\mathbf{N 2}$ & 0.74436 & 0.74193 & 0.73975 & 0.73731 & 0.73337 \\
\hline $\mathbf{O 2}$ & 0.17791 & 0.16595 & 0.15524 & 0.14339 & 0.12448 \\
\hline
\end{tabular}

The mass fractions tabulated above were used in ANSYS FLUENT to create a gas mixture that still used the ideal gas law to calculate density. However, the thermal properties were calculated as shown in Equations 21-23. The temperature depended thermal properties of each species " $i$ " was input as polynomial curve fits as shown in Equation 25 for a generic property $\eta$.

$$
\begin{gathered}
C_{p, \text { mixture }}=\sum_{i=1}^{N} x_{i} C_{p, i} \\
k_{\text {mixture }}=\sum_{i=1}^{N} \frac{x_{i} k_{i}}{\sum_{j=1}^{N} x_{j} \phi_{i j}} \\
\mu_{\text {mixture }}=\sum_{i=1}^{N} \frac{x_{i} \mu_{i}}{\sum_{j=1}^{N} x_{j} \phi_{i j}} \\
\phi_{i j}=\frac{\left[1+\left(\frac{\mu_{i}}{\mu_{j}}\right)^{1 / 2}\left(\frac{M W_{i}}{M W_{j}}\right)^{1 / 4}\right]^{2}}{8\left[1+\left(\frac{M W_{i}}{M W_{j}}\right)^{1 / 2}\right]} \\
\eta(T)=A+B T+C T^{2}+\cdots
\end{gathered}
$$

\subsection{Conduction Error Study Test Case from Ref. 14}

After conducting the validation case, it was desired to apply the computational simulations to an experimental case conducted at Virginia Tech that investigated conduction error in total temperature thermocouple sensors. This work, as previously mentioned, is outlined in Refs. 14-15. However, it was desired to extend the computational work to include the effects of radiation as is done in this study. 


\subsubsection{Geometry and Meshing}

Again, the geometry was first modeled in SolidWorks. The computational domain chosen is shown in Figure 19. Because of the importance of the strut in the study, it was necessary to include it in the CAD model. However, doing so ruled out the possibility of creating an axisymmetric model. Therefore a three-dimensional model of half of the thermocouple and strut was created, again attempting to use the assumed symmetry of the flow field across the airfoil chord line to reduce the size of the mesh. This model can be seen in Figure 20.

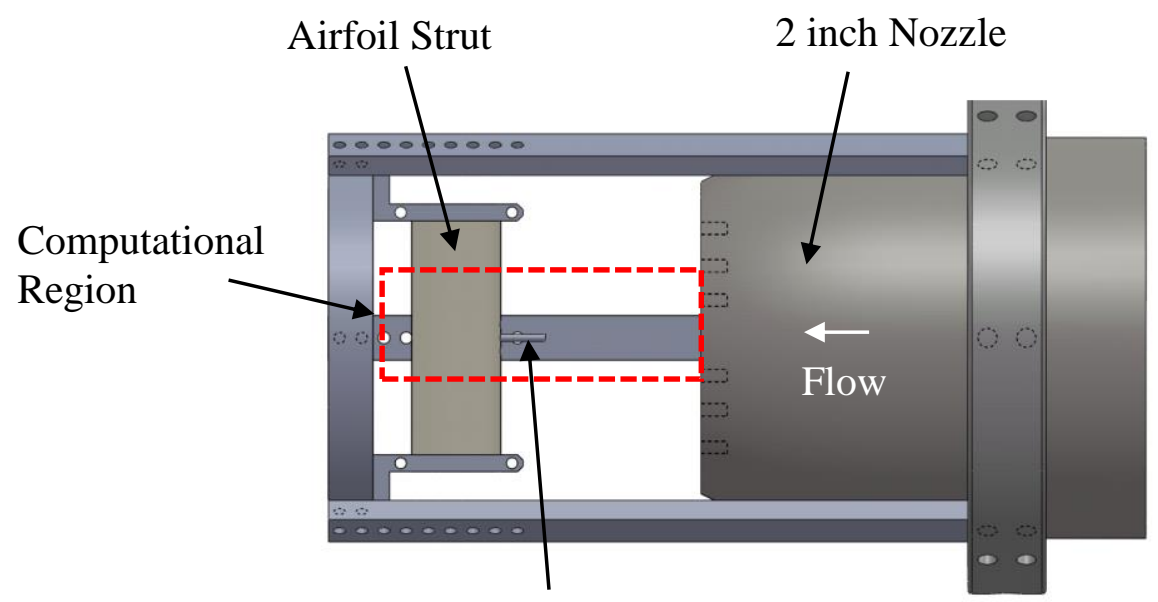

Test Thermocouple Sensor

Figure 19: Computational Domain for Conduction Error Probe Simulations

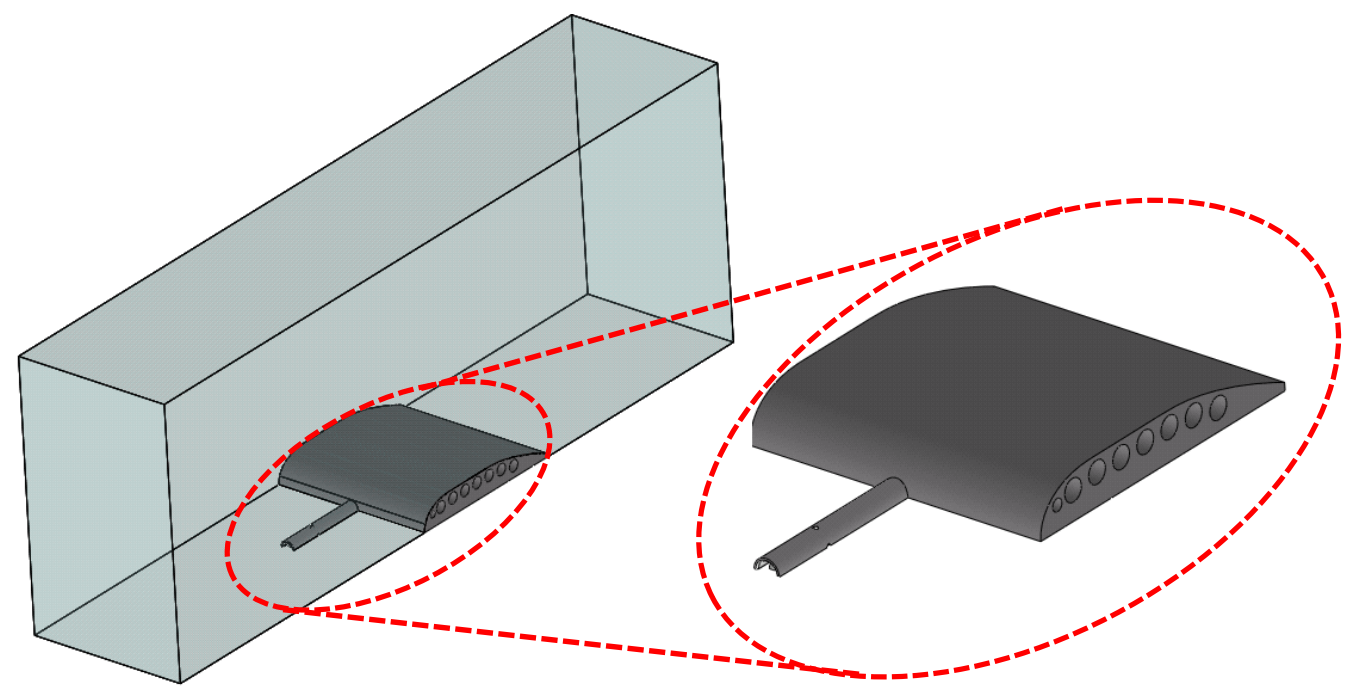

Figure 20: Three-Dimensional Model of Conduction Error Probe

As was done in the validation case, the model was meshed using ANSYS Mechanical. Again an unstructured mesh was developed for both the fluid and solid domains that utilized tetrahedral elements. An inflation layer was created along the solid surfaces to ensure the boundary layer effects were captured. To increase resolution in the area of 
interest, namely the thermocouple junction, a user-defined region of refinement was created. The mesh in the solid domains were sized based on the scale of the domain in which they were located. For example, the mesh within the thermocouple components were quite small whereas the mesh within the strut was larger. This allowed for adequate resolution of the small thermocouple wires but kept the overall mesh size manageable. This mesh contained 1,147,495 elements. For an in depth grid independence study and for more details, the reader is referred to Ref. 15. This mesh can be seen below.

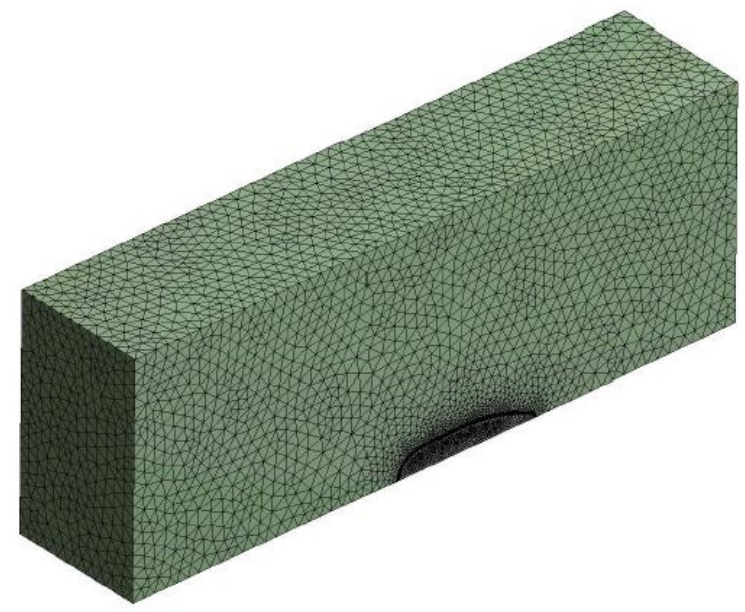

A) Overall Mesh

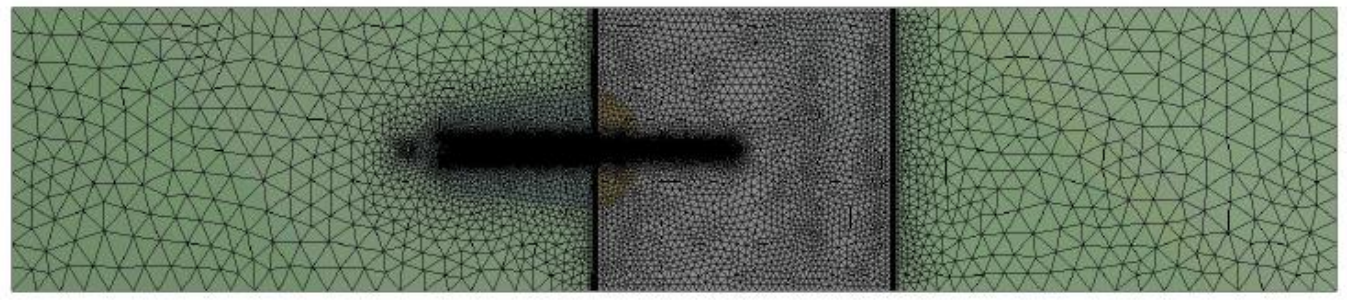

B) View of Probe Region

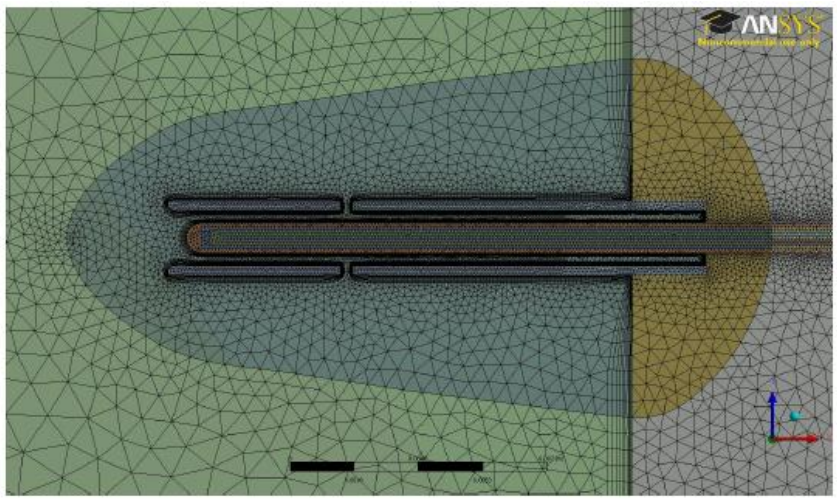

C) Probe Refinement Region

Figure 21: Mesh for Three-Dimensional Conduction Error Probe Simulation [15] 


\subsubsection{Boundary Conditions and Flow Setup}

Simulations were conducted for a range of conditions tested experimentally and documented in Ref. 14. The major parameters that were varied were the total temperature of the flow, the Mach number of the flow, and the non-dimensional conduction driver. Table 5 shows the test matrix developed for the computational study.

Table 5: Test Matrix for Computational Study of Conduction Error

\begin{tabular}{|c|c|c|}
\hline $\begin{array}{c}\text { Total Temperature } \\
\left({ }^{\circ} \mathbf{F}\right)\end{array}$ & Mach Number & $\theta=\left(T_{t}-T_{b}\right) / T_{t}$ \\
\hline \multirow{6}{*}{850} & \multirow{2}{*}{0.8} & 0.5 \\
\hline & & 0.3 \\
\hline & \multirow{2}{*}{0.4} & 0.5 \\
\hline & & 0.3 \\
\hline & \multirow{2}{*}{0.1} & 0.5 \\
\hline & & 0.3 \\
\hline \multirow{6}{*}{550} & \multirow{2}{*}{0.8} & 0.5 \\
\hline & & 0.3 \\
\hline & \multirow{2}{*}{0.4} & 0.5 \\
\hline & & 0.3 \\
\hline & \multirow{2}{*}{0.1} & 0.5 \\
\hline & & 0.3 \\
\hline
\end{tabular}

Again, Pressure Farfield boundary conditions were applied to prescribe the freestream flow conditions for the simulations. Also, the symmetry plane along the center of the airfoil and along the edges of the domain were applied as seen in Figure 22.

The $k$ - $\omega$ SST turbulence model and the Discrete Ordinance (DO) Radiation model were used as was the case in the validation simulations. In these simulations, an upstream radiation source was modeled using an emissivity of 0.2 to represent the radiation from ceramic flow straighteners near the heating coils. The surroundings were also defined as room temperature with an emissivity of 1.0. 


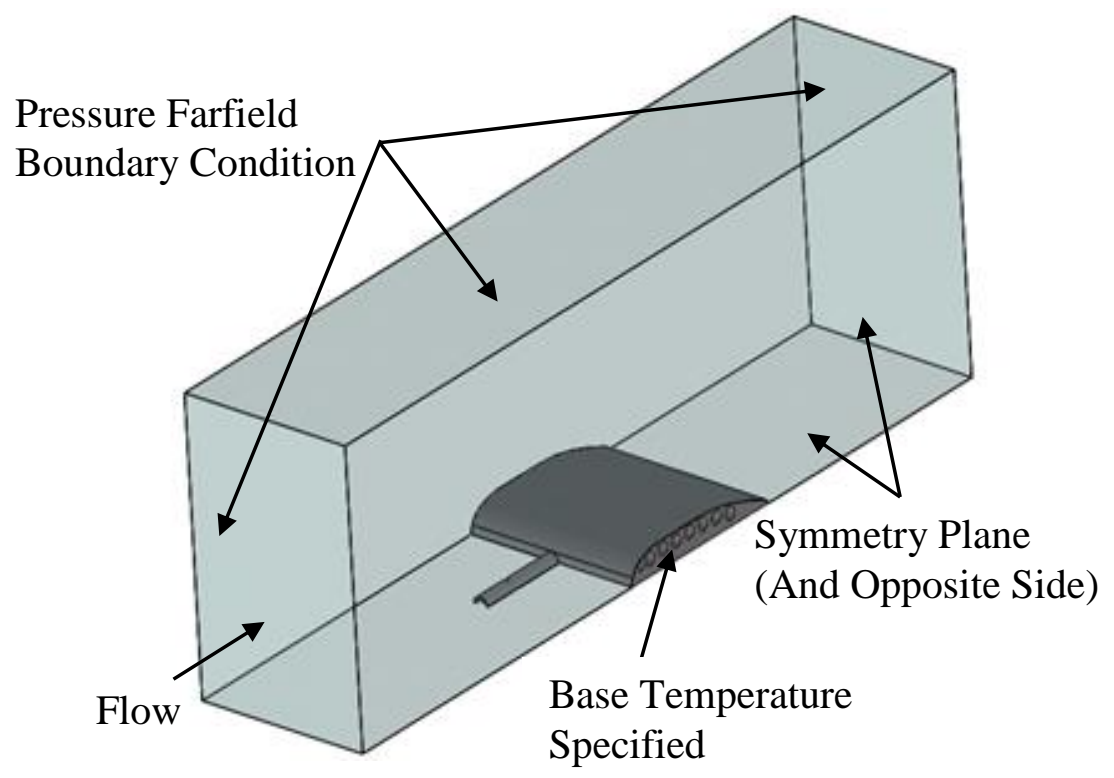

Figure 22: Boundary Conditions for Conduction Error Test Simulations

In modeling the effect of the cooling channels, there were three options: to mesh the channels and model the coolant flowing through them, to prescribe a convective boundary condition at the walls of the cooling channels, or to simply impose the temperature of the coolant directly on the walls of the cooling channels. The first option would have unnecessarily increased computational complexity with little to no true benefit in understanding the performance of the sensor and the assembly, so it was not considered. A simulation was run using a convective boundary condition that specified the convective heat transfer at the walls of the cooling channel based on a representative convective heat transfer coefficient and mean fluid temperature. These results were then compared to the third option of simply prescribing the walls of the cooling channels as the temperature the coolant would need to be to yield a prescribed conduction driver. In both cases, the base temperature and recovery were monitored. Although it was felt that the temperature boundary condition may be a better representation of a liquid coolant as opposed to a gaseous coolant which would have a lower convective heat transfer coefficient, it was found that by simply prescribing the wall temperature, the conduction driver was easily reached. Therefore, this simpler modeling approach was utilized to impose the required base temperature for the desired conduction driver.

In these simulations, the working fluid was indeed heated air as that was what the experimental hot jet facility used. The temperature-dependent properties of air were input into the material definitions based on the tabulated values of Ref. 26. The thermophysical properties of the solid components are shown in Table 6 below. 
Table 6: Thermo-Physical Properties of Thermocouple and Strut Components in Conduction Error Simulations

\begin{tabular}{|c|c|c|c|c|}
\hline Component & Material & $\begin{array}{c}\text { Thermal } \\
\text { Conductivity }\end{array}$ & $\begin{array}{c}\text { Specific Heat at } \\
\text { Constant Pressure }\end{array}$ & Emissivity \\
\hline $\begin{array}{c}\text { Sheath \& } \\
\text { Strut }\end{array}$ & Inconel & $11.5-33.5 \mathrm{~W} / \mathrm{m}-\mathrm{K}$ & $434.1-500.60 \mathrm{~J} / \mathrm{kg}-\mathrm{K}$ & 0.2 \\
\hline Strut TBC & Zirconia & $1 \mathrm{~W} / \mathrm{m}-\mathrm{K}$ & $473.44-669.77 \mathrm{~J} / \mathrm{kg}-\mathrm{K}$ & 0.7 \\
\hline Shield & $\begin{array}{c}\text { Stainless } \\
\text { Steel }\end{array}$ & $26.913 \mathrm{~W} / \mathrm{m}-\mathrm{K}$ & $924.60 \mathrm{~J} / \mathrm{kg}-\mathrm{K}$ & 0.56 \\
\hline Potting & $\begin{array}{c}\text { Magnesium } \\
\text { Oxide }\end{array}$ & $\begin{array}{c}8.0-24.75 \\
\mathrm{~W} / \mathrm{m}-\mathrm{K}\end{array}$ & $963-1398 \mathrm{~J} / \mathrm{kg}-\mathrm{K}$ & $\begin{array}{c}\text { Not } \\
\text { Exposed }\end{array}$ \\
\hline TC & $\begin{array}{c}\text { Chromel- } \\
\text { Alumel }\end{array}$ & $24.45 \mathrm{~W} / \mathrm{m}-\mathrm{K}$ & $485.5 \mathrm{~J} / \mathrm{kg}-\mathrm{K}$ & $\begin{array}{c}\text { Not } \\
\text { Exposed }\end{array}$ \\
\hline
\end{tabular}

\section{Computational Results}

The results of the computational simulations for the validation case are presented first. These results will be compared to the experimental findings of the validation case given in Ref. 10. After validating the approach, the results of the conduction error simulations will be given and compared to the appropriate experimental results.

\subsection{Validation Case Results}

First, validation results with respect to aerodynamic corrections at low temperatures were investigated by Schneider and more information can be found in Refs. 12 and 15. A brief review of these results will be given here for completeness. Second, to validate the radiation model and conjugate heat transfer, the empirical radiation correction developed by Glawe et al. and given in Equation 4 was compared with the radiation correction obtained from the simulations.

\subsubsection{Aerodynamic Error Comparisons}

A validation for the use of CFD to predict the aerodynamic recovery of a thermocouple at low temperatures was conducted by Schneider in Ref. 15. Simulations were undertaken at a total temperature of $540 \mathrm{R}(300 \mathrm{~K})$ and total pressures of $1 \mathrm{~atm}$ and $20 \mathrm{~atm}$ with the Mach number varying between 0.3 and 0.9 . This was done to attempt to match the result presented by Glawe shown in Figure 6 and Figure 7. It was shown that the simulation results for the aerodynamic recovery followed the same trend as that of the experimental results. In particular, good quantitative agreement was found between the threedimensional, shielded model and the experimental results. However, the two-dimensional models, particularly the unshielded probe model, did not compare quite as well quantitatively. This was most likely due to the three-dimensional nature of the flow over the junction of the thermocouple not being captured by the two-dimensional rod model of 
the thermocouple that was used $[12,15]$. Also, the discrepancies between the twodimensional, axisymmetric results could be attributed to the assumptions made during the geometry development.

An in-depth presentation of these validation results can be found in Ref. 15, but overall, the results validated the use of CFD to predict the performance of total temperature thermocouple sensors at low temperatures.

\subsubsection{Radiation Error Comparisons}

To extend this validation to higher temperatures with a focus on radiation modeling, simulations were undertaken at total temperatures of 1600R $(888.89 \mathrm{~K}), 1800 \mathrm{R}(1000 \mathrm{~K})$, 2000R (1111.11K), 2200R (1222.22K), and 2500R (1388.89K) and a Mach number of 0.3. These conditions were chosen directly from the results presented in Ref. 10 .

Typical results from the simulations can be seen in Figures 23-26. These include the temperature distributions in the thermocouple and the surrounding gas as well as the radiative heat fluxes at the surface of the thermocouple. In the radiative heat transfer plots, positive values indicate radiation emitted from the thermocouple whereas negative values indicate radiation absorbed by the assembly. Figure 23 and Figure 24 show the results from the two-dimensional, axisymmetric simulation of the unshielded probe at a total temperature of $2500 \mathrm{R}(1388.89 \mathrm{~K})$. It is evident from these figures that junction of the thermocouple loses a large quantity of heat due to radiation as indicated by the cooler temperatures and the large amounts of emitted radiation.

Figure 25 and Figure 26 show the same results for the three-dimensional model of the shielded probe. Again, it is seen that the exposed junction is at a lower temperature than the true total temperature of the flow as well as the body of the thermocouple due to radiation heat transfer to the cooler surroundings. However, comparing the contours of temperature and radiative heat flux between those for the unshielded probe, it can be seen that the shield has significantly reduced the effect of this radiation heat transfer. This is better quantified in Figure 27 which shows the radiative heat flux along the length of the thermocouple from the axisymmetric models. The $\mathrm{x}$-axis represents the length from the tip of the thermocouple moving towards the housing. It is clearly evident that the shield limits the radiative heat loss to the surroundings. Also visible is the sharp increase in radiative heat flux at the tip of the shielded probe, where it is exposed at the entrance of the shield. In these cases, the temperature indicated by the thermocouple was $1308 \mathrm{~K}$ and $1258 \mathrm{~K}$, for the shielded and unshielded probes, respectively. Again, this is a large difference from the true total temperature of $1388.89 \mathrm{~K}$ and is almost entirely due to radiation effects. 


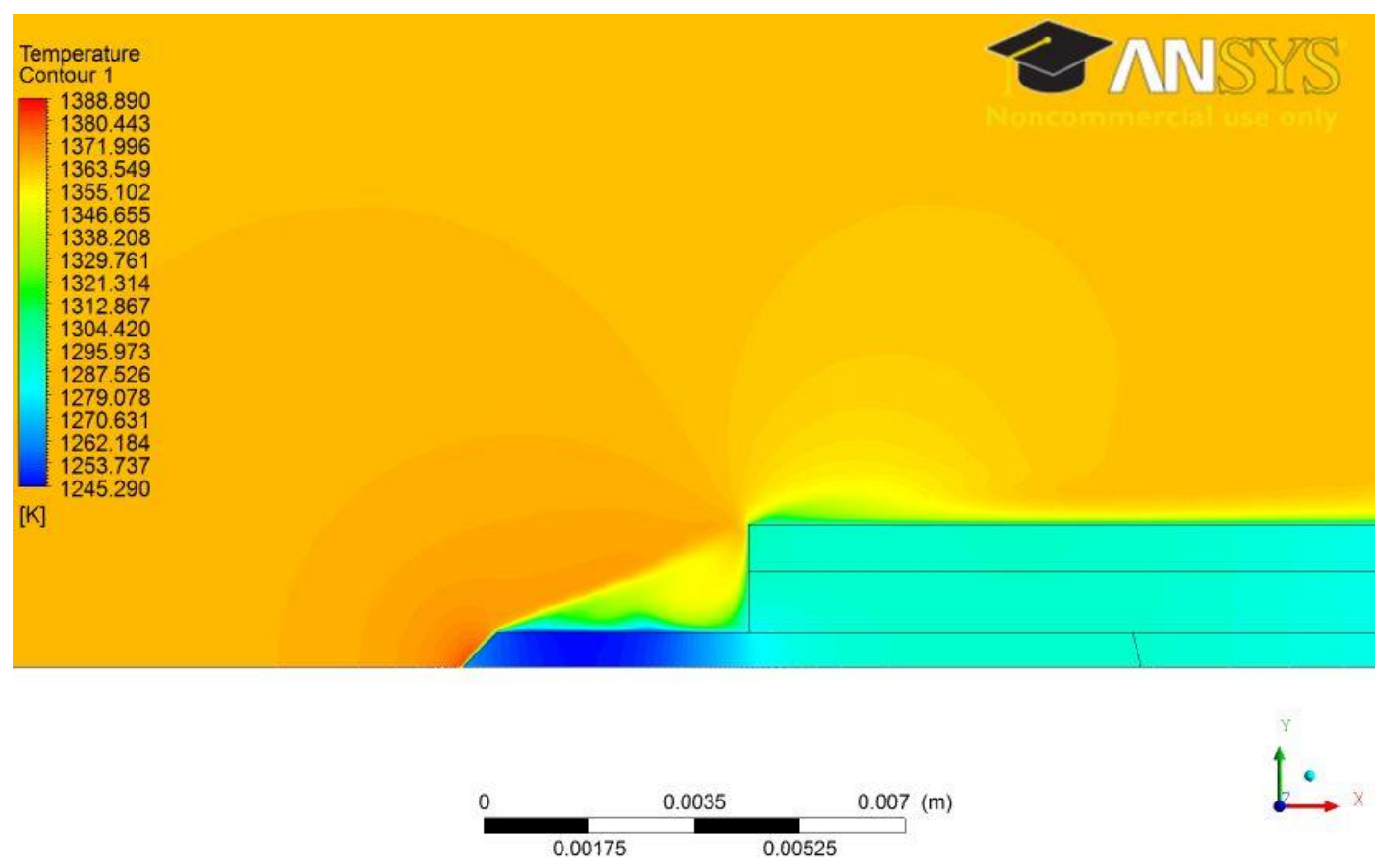

Figure 23: Predicted Temperature Distribution for Two-Dimensional, Axisymmetric Model of Unshielded Probe from Ref. 10 at Flow Total Temperature of 2500R and Mach Number of 0.3

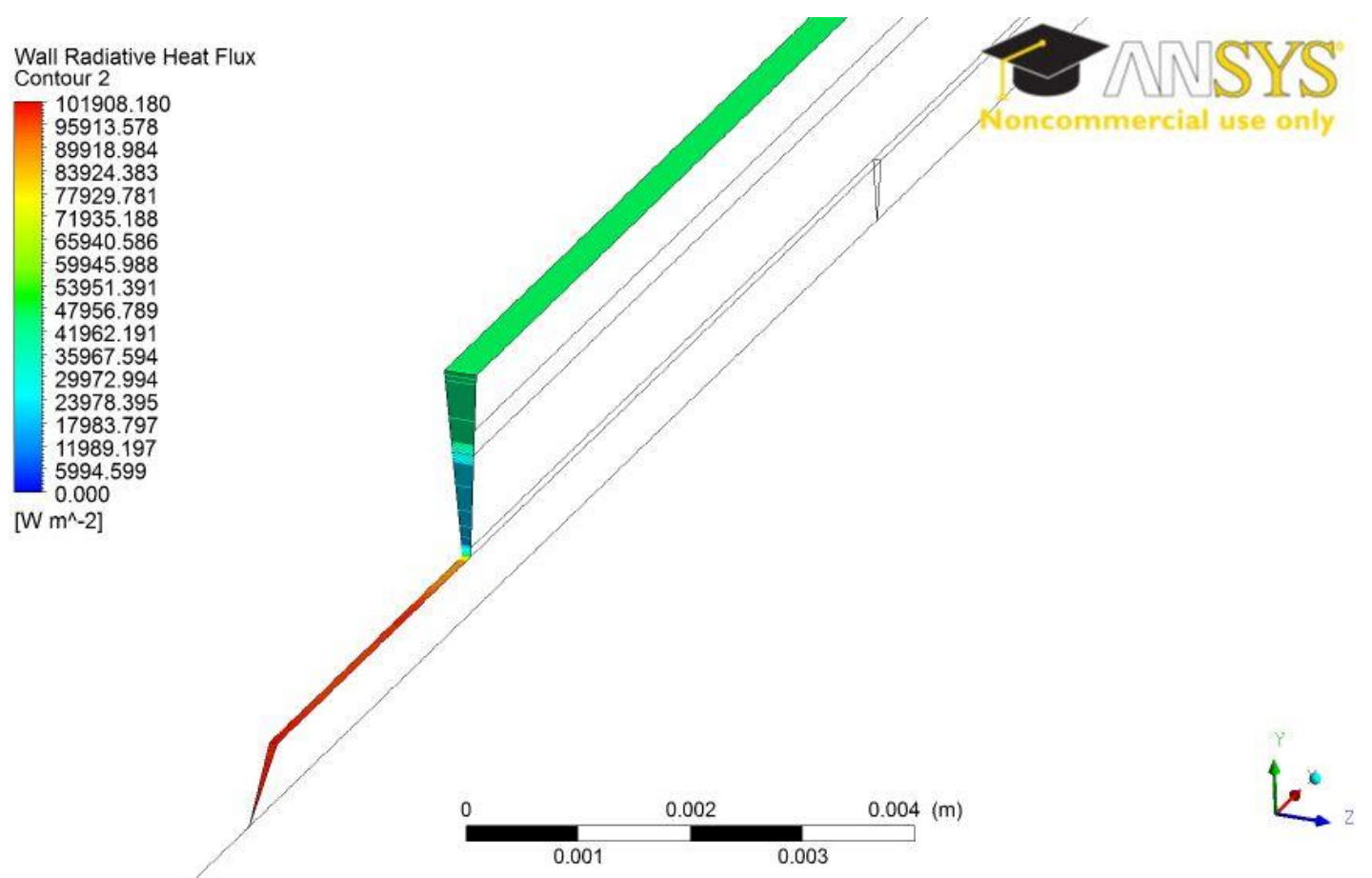

Figure 24: Predicted Radiative Heat Flux Distribution for Two-Dimensional, Axisymmetric Model of Unshielded Probe from Ref. 10 at Flow Total Temperature of 2500R and Mach Number of 0.3 


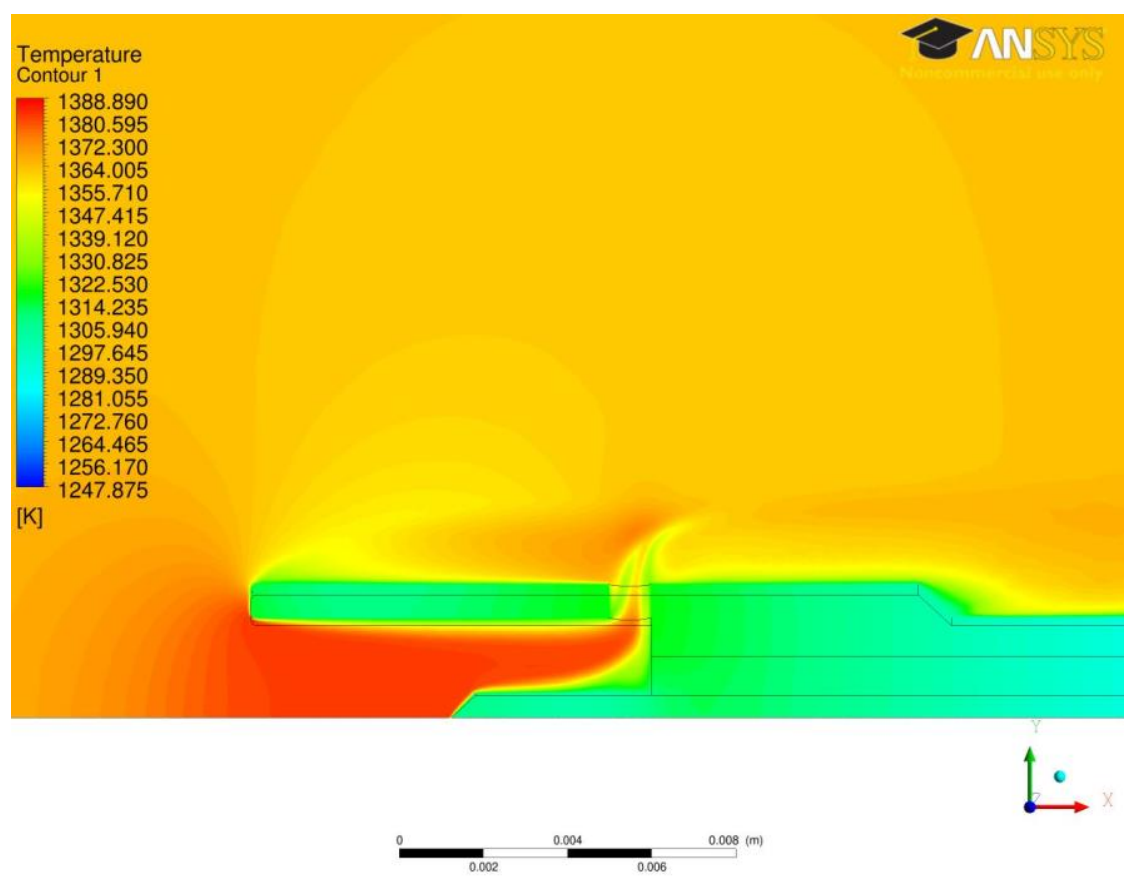

Figure 25: Predicted Temperature Distribution for Three-Dimensional Model of Shielded Probe of Ref. 10 at Flow Total Temperature of 2500R and Mach Number of 0.3

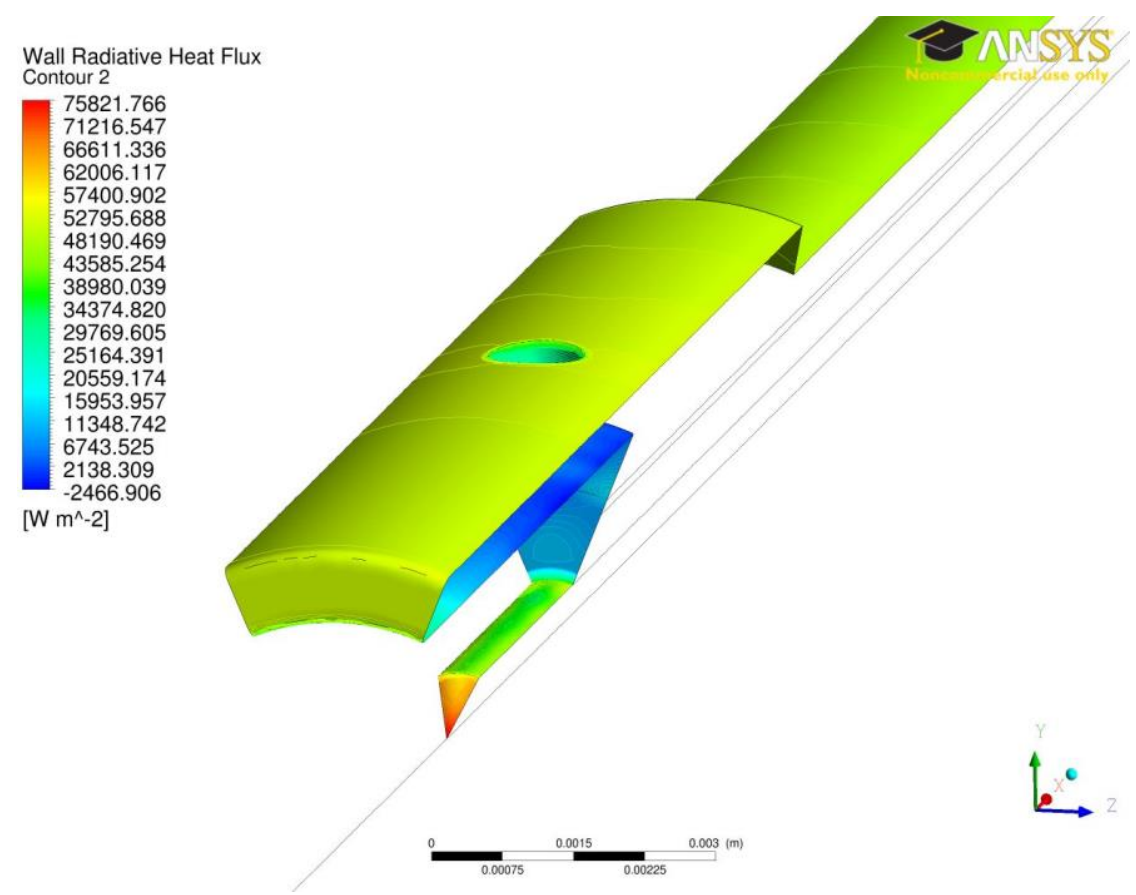

Figure 26: Predicted Radiative Heat Flux Distribution for Three-Dimensional Model of Shielded Probe of Ref. 10 at Flow Total Temperature of 2500R and Mach Number of 0.3 


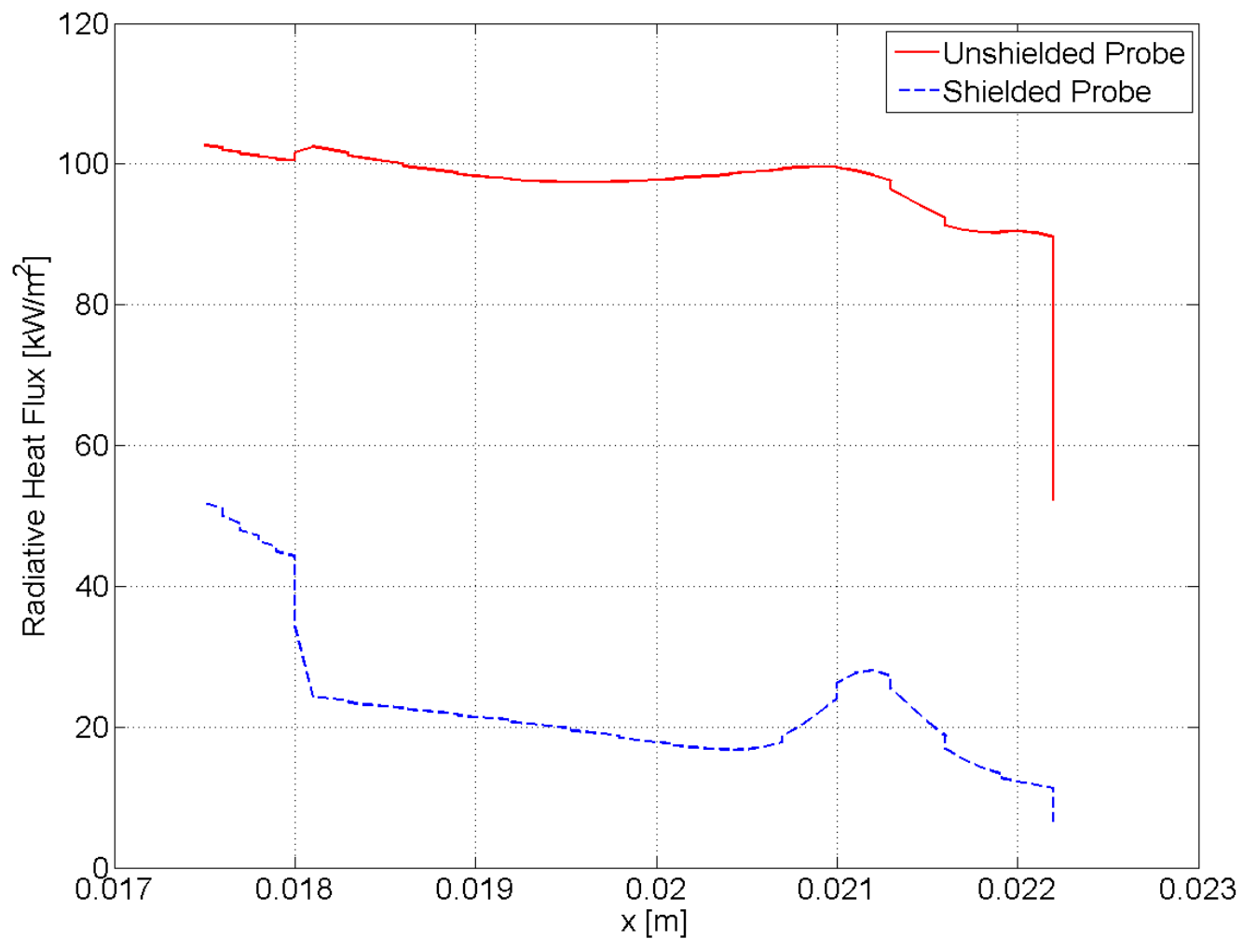

Figure 27: Radiative Heat Flux from Exposed Thermocouples from the Two-

Dimensional, Axisymmetric Models at Flow Total Temperature of 2500R and Mach Number of 0.3

The empirical radiation correction developed in Ref. 10 and given in Equation 4 was plotted and compared to the radiation corrections obtained from the simulations. To obtain the radiation correction from the simulations, the total temperature that would be measured by the thermocouple was extracted from the simulations by averaging the temperature in a conical volume at the tip of thermocouple rod where the junction would be located. This value was first corrected with the aerodynamic correction given in Ref. 10. It should be noted that this correction was quite small for a Mach number of 0.3 due to the probes' design. Once the junction temperature was corrected for aerodynamic errors, its value was subtracted from the true total temperature of the flow to yield the radiation correction. This procedure follows the experimental procedure and was done for the shielded and unshielded probes as plotted in Figure 28. In this figure, the radiation correction is plotted as a function of the indicated junction temperature of the thermocouple. The solid red curve is the radiation correction for the unshielded probe, and the broken blue curve is the radiation correction for the shielded probe as given by Equation 4 and documented in Ref. 10. The asterisks indicate the results of the twodimensional axisymmetric simulations using air as the working fluid. The diamonds indicate the results of the two-dimensional axisymmetric simulations using the gas mixture of combustion products as the working fluid. Finally, the solid circles are the results of the three-dimensional simulations of the shielded probe with air as the working fluid. 


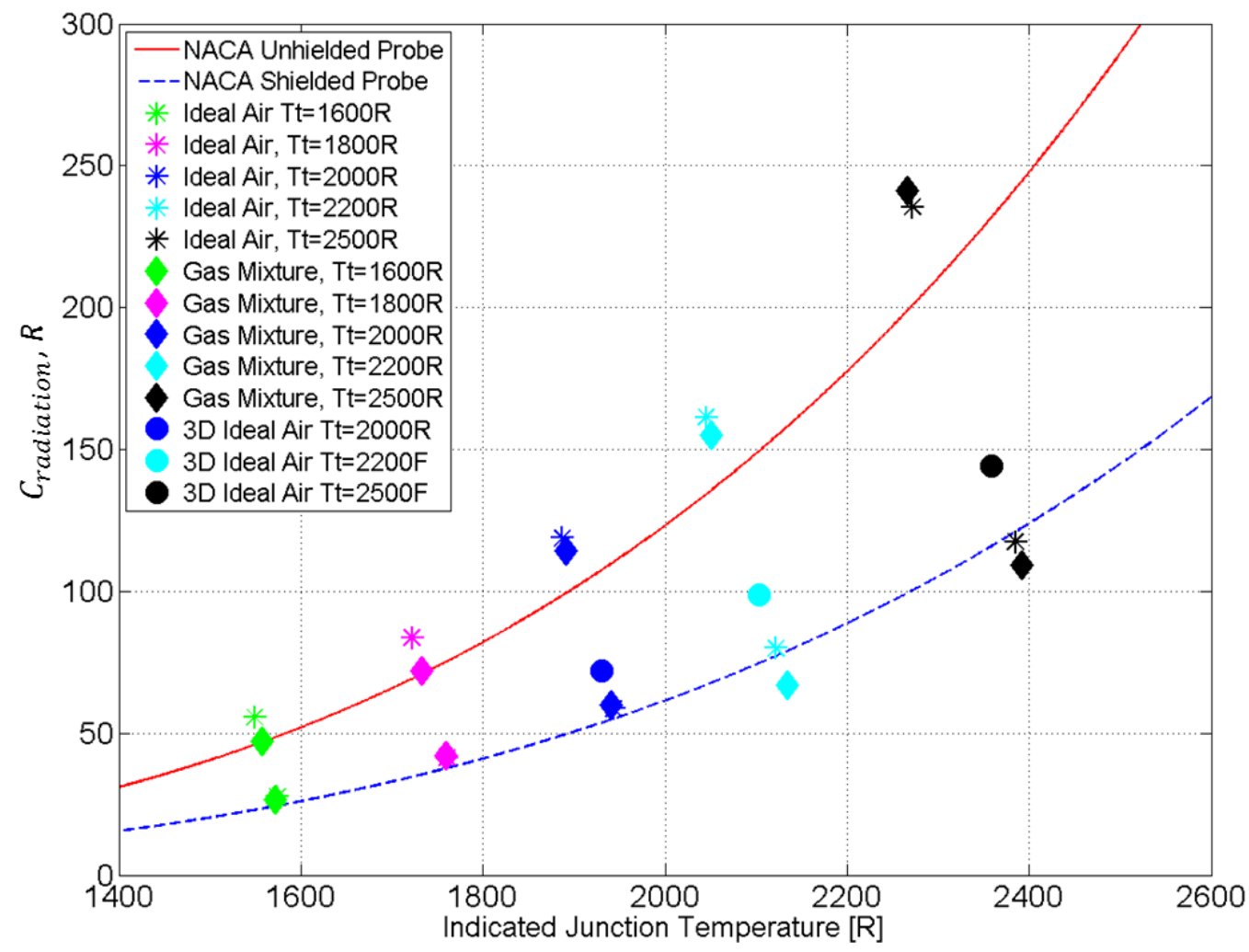

Figure 28: Comparison of Radiation Corrections from Current Simulations with Empirical Correlation from Ref. 10

The results compare well with the experimental results of Ref. 10 following the same trend of increasing as the total temperature of the flow increases. This is because as the flow total temperature increased, the junction of the thermocouple increased in temperature as well, leading to a larger temperature difference between it and the surroundings causing higher radiative heat fluxes. The effect of the fourth power in radiation heat transfer formulations is also clearly visible in the results, again as the temperature increases, the radiation correction rapidly increases [12]. Relevant statistics between the CFD predicted radiation corrections and those tabulated in the NACA report are given below.

Table 7: RMS Error and Percent Difference between Predicted Radiation Corrections and Reported Data from Ref. 10

A) Three-Dimensional, Shielded Model

\begin{tabular}{|c|c|c|c|c|}
\hline Junction Temp (R) & $\begin{array}{c}\text { CFD Radiation } \\
\text { Correction (R) }\end{array}$ & $\begin{array}{c}\text { NACA } \\
\text { Radiation } \\
\text { Correction (R) }\end{array}$ & $\begin{array}{c}\text { RMS Error } \\
\text { (R) }\end{array}$ & $\begin{array}{c}\text { Percent } \\
\text { Difference (\%) }\end{array}$ \\
\hline 1929.89 & 72.1 & 53.67 & 18.43 & 29.31 \\
\hline 2103.457 & 98.74 & 74.71 & 24.03 & 27.71 \\
\hline 2358.196 & 144.3 & 115.80 & 28.5 & 21.91 \\
\hline
\end{tabular}


B) Two-Dimensional, Axisymmetric, Shielded Model

\begin{tabular}{|c|c|c|c|c|}
\hline $\begin{array}{c}\text { Junction Temp } \\
\text { (R) }\end{array}$ & $\begin{array}{c}\text { CFD Radiation } \\
\text { Correction (R) }\end{array}$ & $\begin{array}{c}\text { NACA } \\
\text { Radiation } \\
\text { Correction (R) }\end{array}$ & $\begin{array}{c}\text { RMS Error } \\
\text { (R) }\end{array}$ & $\begin{array}{c}\text { Percent } \\
\text { Difference (\%) }\end{array}$ \\
\hline \multicolumn{5}{|c|}{ Air Results } \\
\hline 1574.02 & 27.58 & 24.44 & 3.14 & 12.06 \\
\hline 1760.44 & 41.36 & 37.68 & 3.68 & 9.32 \\
\hline 1943.30 & 58.68 & 55.12 & 3.56 & 6.26 \\
\hline 2122.14 & 80.05 & 77.29 & 2.76 & 3.51 \\
\hline 2385.11 & 117.39 & 120.95 & 3.56 & -2.98 \\
\hline \multicolumn{5}{|c|}{ Gas Mixture Results } \\
\hline 1572.96 & 26.64 & 24.38 & 2.26 & 8.86 \\
\hline 1759.64 & 42.16 & 37.61 & 4.55 & 11.41 \\
\hline 1941.90 & 60.06 & 54.96 & 5.10 & 8.86 \\
\hline 2135.30 & 66.88 & 79.14 & 12.26 & -16.79 \\
\hline 2393.11 & 109.39 & 122.51 & 13.12 & -11.31 \\
\hline
\end{tabular}

C) Two-Dimensional, Axisymmetric, Unshielded Model

\begin{tabular}{|c|c|c|c|c|}
\hline $\begin{array}{c}\text { Junction Temp } \\
\text { (R) }\end{array}$ & $\begin{array}{c}\text { CFD Radiation } \\
\text { Correction (R) }\end{array}$ & $\begin{array}{c}\text { NACA } \\
\text { Radiation } \\
\text { Correction (R) }\end{array}$ & RMS Error (R) & $\begin{array}{c}\text { Percent } \\
\text { Difference } \\
\text { (\%) }\end{array}$ \\
\hline \hline \multicolumn{5}{|c|}{ Air Results } \\
\hline 1549.27 & 55.53 & 45.97 & 9.56 & 18.84 \\
\hline 1721.78 & 83.62 & 69.17 & 14.45 & 18.91 \\
\hline 1887.22 & 118.78 & 98.49 & 20.28 & 18.67 \\
\hline 2045.20 & 161.38 & 134.15 & 27.24 & 18.43 \\
\hline 2271.99 & 235.51 & 200.80 & 34.71 & 15.91 \\
\hline \multicolumn{5}{|c|}{ Gas mixture } \\
\hline 1557.75 & 47.05 & 46.96 & 0.09 & 0.19 \\
\hline 1733.60 & 71.80 & 71.02 & 0.78 & 1.10 \\
\hline 1891.52 & 114.48 & 99.36 & 15.12 & 14.14 \\
\hline 2051.60 & 154.99 & 135.76 & 19.23 & 13.22 \\
\hline 2266.30 & 241.20 & 198.88 & 42.32 & 19.23 \\
\hline
\end{tabular}

In analyzing these results and looking at the RMS error, it can be seen that the radiation corrections of the simulations compare well at low temperatures but begin to depart the experimental curve as the temperature continues to increase. The reason for this is that, as mentioned in Section 4.1.2, the surrounding temperature was defined as constant at room temperature (540R or $300 \mathrm{~K})$. This surrounding temperature was to represent the surrounding walls of the tunnel. The temperature was specified to be room temperature because no information of the temperature that the duct reached was given in Ref. 10. 
However, in reality, it is likely that as the temperature of the flow increased, the wall temperature also increased. This would then reduce the radiation heat transfer from the junction of the thermocouple to the walls thus reducing the radiation correction.

Therefore, if the duct temperature was modeled more accurately, it is believed that the predicted radiation corrections at higher temperatures would compare better with the experimental results. It should also be noted that this effect is more apparent in the results for the unshielded probe, the reason being that the shield reduces the solid angle or view factor that the hot thermocouple has to "see" the cooler surroundings. Another source of discrepancy is the uncertainty in the emissivity values used. For example, in these simulations, the emissivity was defined as constant. However, there has been work that suggests that the emissivity, particularly that of Inconel, increases as the temperature increases [29]. Despite this, it is believed that the results show good agreement even without this information, which could only be obtained through comprehensive experimental tests.

Next, it was desired to compare the results between the simulations that used air as the working fluid and the simulations that used the combustion products mixture. The difference between the results are small, especially at low temperatures. As the temperature of the flow increased, there was a slight difference in the results, but it was still quite small. Again, this justified the initial assumption that the combustion was lean and the products dilute and could be accurately modeled as air.

Finally, the results of the two-dimensional, axisymmetric simulations and the threedimensional simulations of the shielded probe were compared. One can observed that the three-dimensional simulation results actually have a higher radiation correction than the two-dimensional models. The reason for this is that the "slot" in the two-dimensional model was sized to have the same mass flow rate as the vent holes of the actual probe when at a Mach number of 0.6. Therefore, it is likely that the slot was oversized when used at a Mach number of 0.3. This would imply that a greater mass flow rate existed over the junction of the thermocouple increasing the convective heat transfer to the junction and reducing the effect of radiation heat transfer from the junction.

\subsection{Conduction Error Test Case Results}

After validating the computational methods by comparisons with the legacy experimental work, it was desired to apply these methods to the experimental conduction error study being performed at Virginia Tech. This study focused on analyzing the effects of conduction error in total temperature thermocouple sensors both experimentally and computationally.

\subsubsection{Radiation Effects Results}

In these results, it was assumed that the primary source of error in the thermocouple reading was the induced conduction error. However, it was desired to investigate the effects of radiation as well. To investigate the effects of radiation on the recovery, simulations were run with and without the radiation model. A comparison between the 
recovery values with and without modeling radiation are tabulated in Table 8 . As can be seen, the effects of radiation are quite negligible. This is, of course, due to the modest $\left(<850^{\circ} \mathrm{F}\right)$ temperatures that were used in the experiment and modeled in the simulation. This was important to quantify as the experimental work was investigating solely conduction error, and these results prove that radiation played a minor role in the overall performance of the sensors.

Table 8: Recovery Values from Simulations with and without Radiation Modeling

\begin{tabular}{|c|c|c|c|c|}
\hline \multicolumn{3}{|c|}{ Simulated Case } & \multirow[b]{2}{*}{$\begin{array}{l}\text { Recovery with } \\
\text { Radiation }\end{array}$} & \multirow[b]{2}{*}{$\begin{array}{l}\text { Recovery without } \\
\text { Radiation }\end{array}$} \\
\hline $\begin{array}{c}\text { Total Temperature } \\
\left({ }^{\circ} \mathbf{F}\right)\end{array}$ & $\theta=\frac{\left(T_{t}-T_{b}\right)}{T_{t}}$ & $\mathbf{M}$ & & \\
\hline \multirow{2}{*}{850} & \multirow{2}{*}{0.5} & 0.8 & 0.9806 & 0.9813 \\
\hline & & 0.1 & 0.9124 & 0.9196 \\
\hline \multirow{2}{*}{550} & \multirow{2}{*}{0.5} & 0.8 & 0.9846 & 0.9848 \\
\hline & & 0.1 & 0.921 & 0.9232 \\
\hline
\end{tabular}

Typical contours of radiative heat flux for the entire assembly and the thermocouple surface, as well as temperature contours for the assembly are shown in Figure 29, Figure 30 and Figure 31, respectively. Specifically, the results for the case with a total temperature of $850^{\circ} \mathrm{F}(727 \mathrm{~K})$, Mach number of 0.8 , and conduction driver of 0.5 is shown. In Figure 29 and 30, the positive values of heat flux indicate that radiation is being emitted while negative values indicate that heat flux is being absorbed. Note that the maximum radiation heat flux being emitted is located along the surface of the shield. This is because the flow is at nearly the stagnation temperature making the shield hot compared to the surrounding temperature. Also, the shield, being made of stainless steel, has a high emissivity value. The majority of the strut is emitting radiation as it is warmer than the surroundings, despite the large cooling effect. The forward facing region of the strut shows a smaller net emission of radiation, because this region is absorbing heat from the upstream radiation source used to model the flow straightener.

In particular, knowledge of the radiation heat transfer at the surface of the thermocouple is critical. Figure 30 shows that, as expected, radiation is being emitted by the tip of the thermocouple sheath. However, the magnitude of this emitted radiation is much less than that of the shield because the sheath, made of Inconel, has a much lower emissivity than the stainless steel shield. Also, the thermocouple's view factor to the cooler surroundings is being blocked by the presence of the shield. This net radiation emission is the source of the radiation error in thermocouple readings; however, it has previously been shown that at the conditions of these experiments and simulations the radiation error is negligible.

Figure 31 shows the temperature contours over the sensor-strut assembly. Again, the shield and thermocouple are the hottest points due to the stagnation of the flow. The large conduction driver is evident in this figure, and the effects of conduction can be seen in the temperature contours on the shield. The effects of the cooling channels on the temperature distribution throughout the strut is also quite visible. Finally, Figure 32, 
shows temperature distribution in the sheath and the thermocouple wire. The effects of conduction error on the thermocouple measurement are clearly visible here.

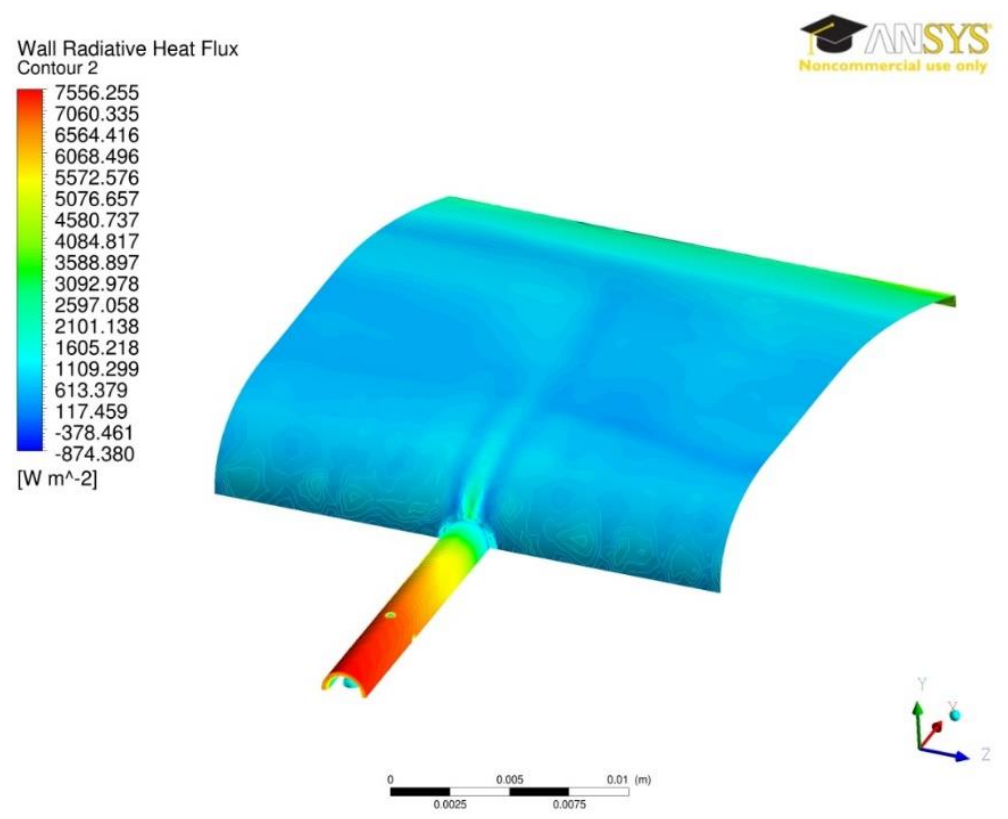

Figure 29: Radiative Heat Flux Contours for Conduction Error Probe at Total

Temperature of $850^{\circ} \mathrm{F}(727 \mathrm{~K})$, Mach Number of 0.8 , and Conduction Driver of 0.5

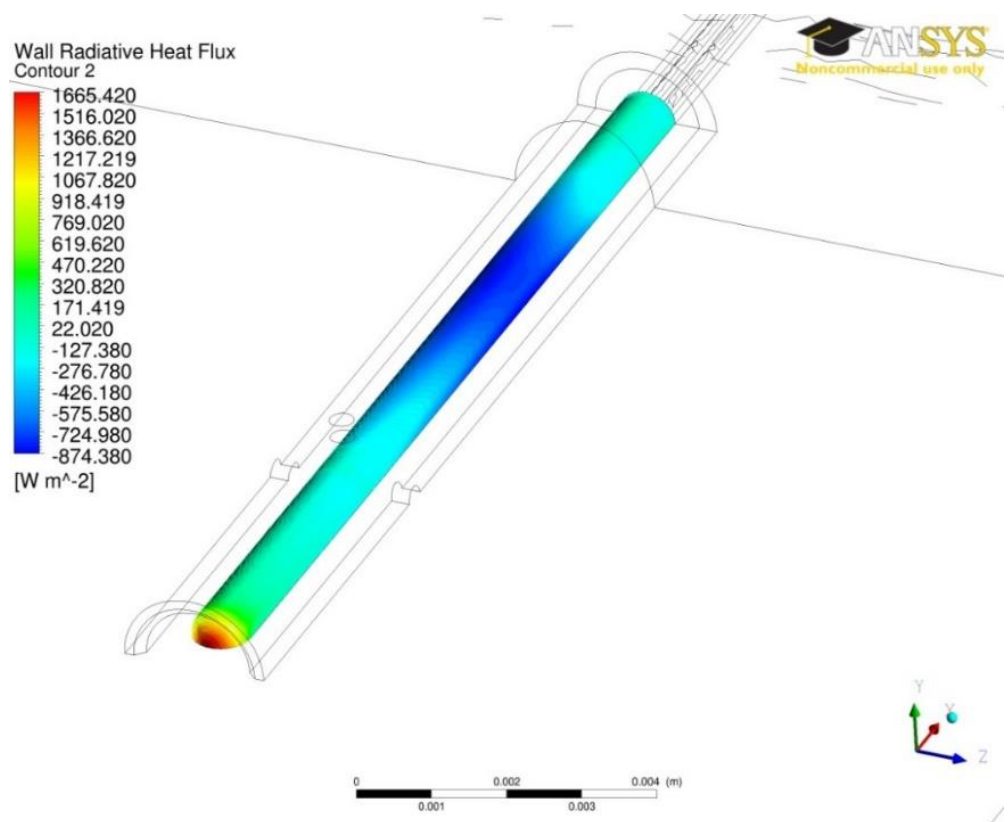

Figure 30: Radiative Heat Flux Contours along Thermocouple Surface for Conduction Error Probe at Total Temperature of $850^{\circ} \mathrm{F}(727 \mathrm{~K})$, Mach Number of 0.8 , and Conduction Driver of 0.5 


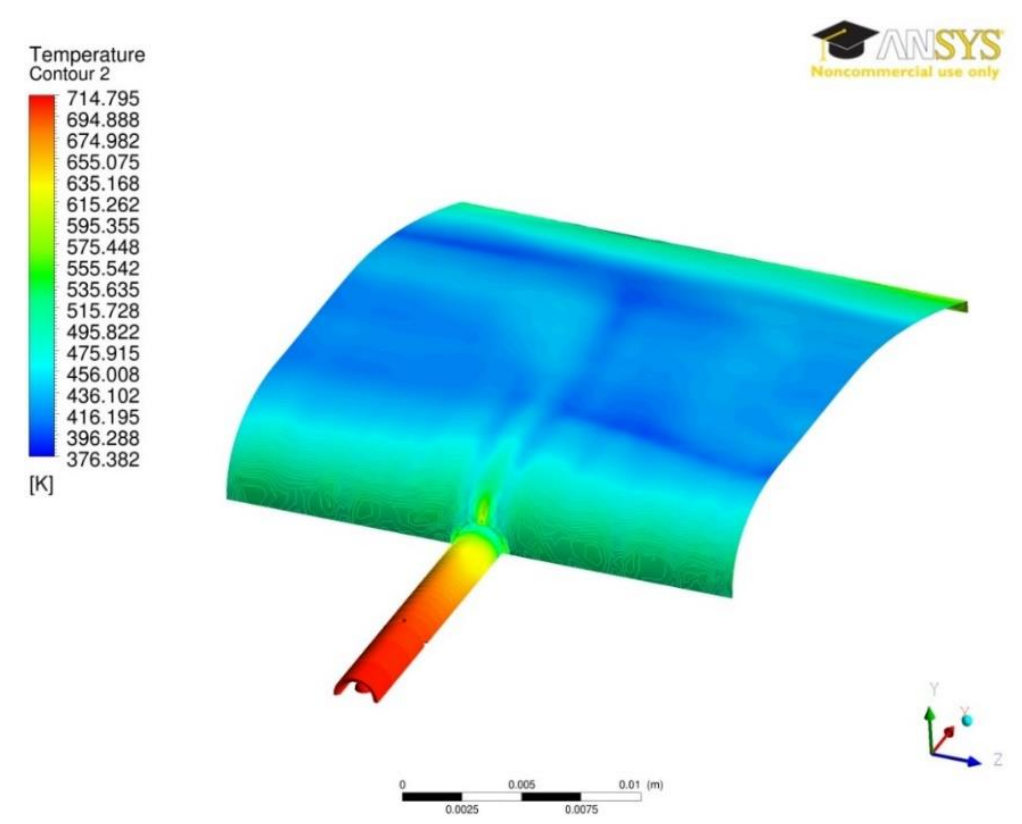

Figure 31: Temperature Contours for Conduction Error Probe at Total Temperature of $850^{\circ} \mathrm{F}(727 \mathrm{~K})$, Mach Number of 0.8 , and Conduction Driver of 0.5

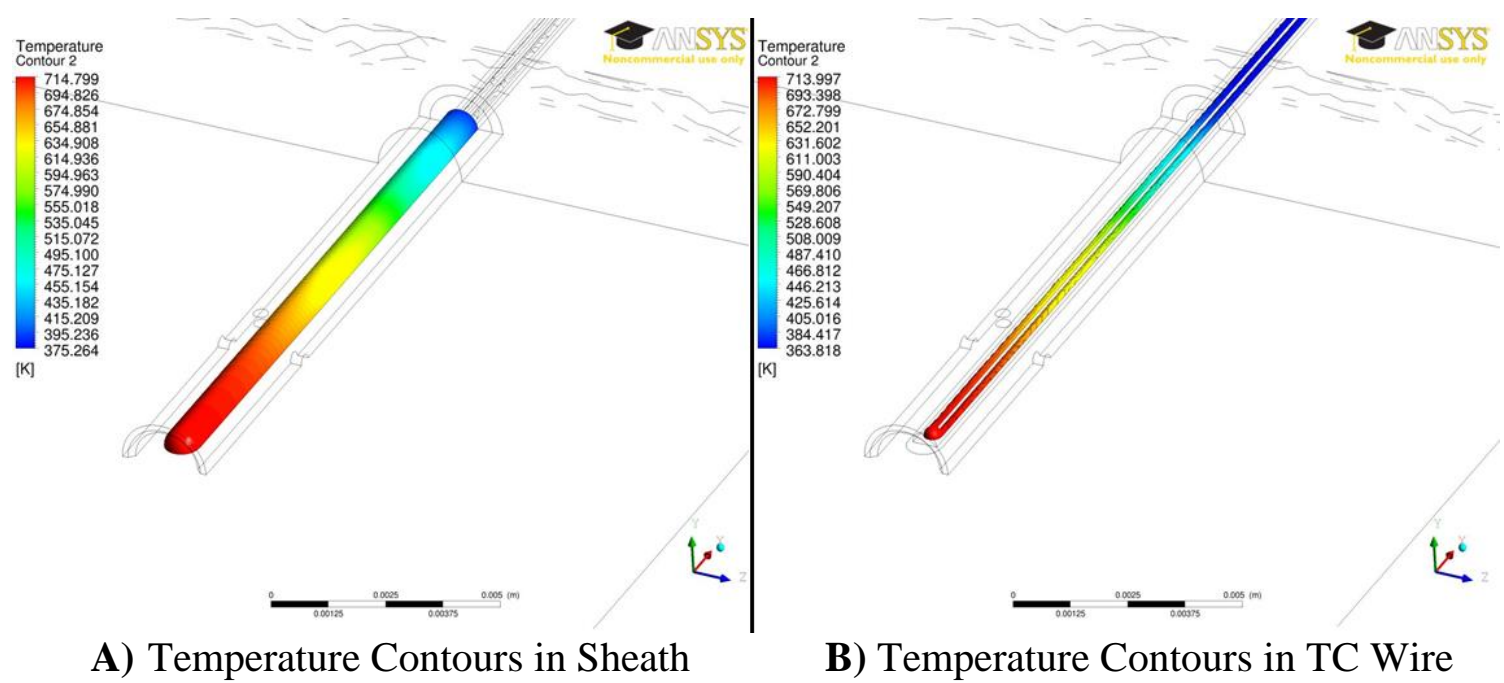

Figure 32: Temperature Contours in Sheath and TC Wire for Conduction Error Probe at Total Temperature of $850^{\circ} \mathrm{F}(727 \mathrm{~K})$, Mach Number of 0.8 , and Conduction Driver of 0.5

\subsubsection{Sensor Recovery Results}

The experimental study focused on the recovery of the thermocouple defined in Equation 6 for a variety of non-dimensional conduction drivers. The recovery for the straight-tube shielded probe used in the experiments are shown in Figure 33. The asterisks indicate the experimental data at total temperatures of $850^{\circ} \mathrm{F}$ and $550^{\circ} \mathrm{F}$ and conduction drivers of 0.6 and 0.5 , respectively. The results of the computational simulations at the same total 
temperatures are shown by the solid circles and diamonds for drivers of 0.5 and 0.3 , respectively.

If the thermocouple was indicating the exact total temperature of the flow, the recovery would be unity. As can be seen, the recovery is low at low Mach numbers but tends to increase as the Mach number is increased. This is because as the flow speed increases, the convective heat transfer coefficient increases. Therefore, at low Mach numbers, the effect of conduction is more dominant than at the higher Mach numbers, leading to a lower recovery value.

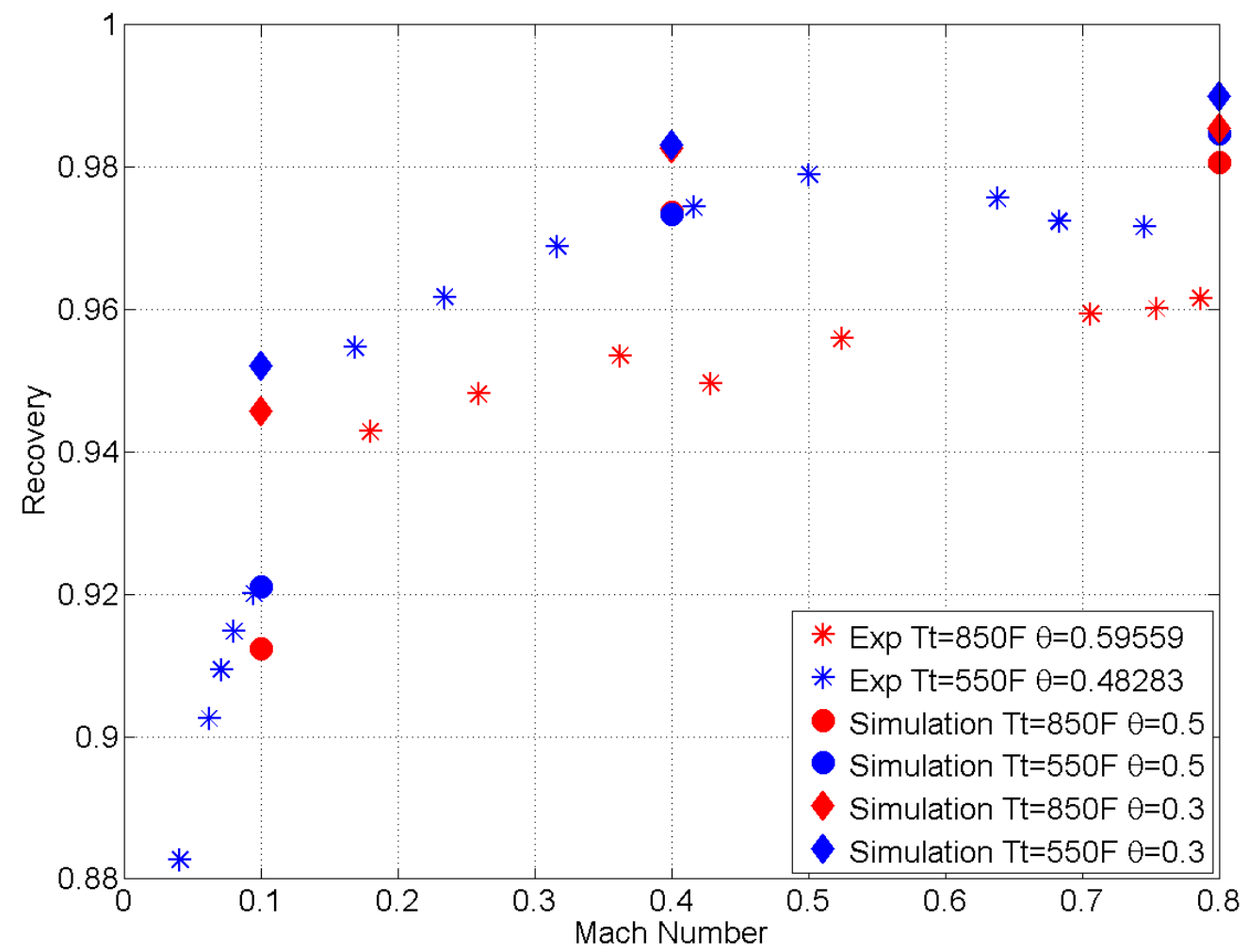

Figure 33: Recovery as a Function of Mach Number for Conduction Error ProbeExperimental Data [14] and Current Predictions

The computational results compare well with the experimental results, especially for the case with a total temperature of $550^{\circ} \mathrm{F}$. It should be noted that after conducting the experiment, it was found that a significant amount of heat may be leaving the strut via conduction through the clamping mechanisms. This possibility is the subject of ongoing investigation but would account for the fact that the simulations yielded higher recovery values than those obtained from the experiments, especially at higher temperatures and lower drivers where the strut would be much warmer. In particular, this is possible because the simulation model did not include the clamping mechanism and only modeled a portion of the strut in the flow, thus not allowing for heat transfer to leave at the strut at the boundaries. 
In addition, the recovery results were plotted as a function of the Reynolds number as it plays an important role in the overall heat transfer processes. Specifically, the convective heat transfer to the thermocouple can be related to the flow physics by using the Reynolds Analogy which relates the skin friction coefficient to the Nusselt or Stanton number [12]. In Figure 34, the recovery results are plotted as a function of the Reynolds number based on the properties and velocity of the freestream and the diameter of the thermocouple (0.032 in). Again, the good agreement between the simulated results and the experimental results can be noted.

Also, as discussed in Ref. 14 and elsewhere, the recovery should be independent of the total temperature of the flow, and should only depend on the Reynolds number and the conduction driver, the two pertinent non-dimensional numbers. This indeed can be seen in the results, where, for the same conduction driver, the results for the two total temperatures seem to align well. This is significant in that it further validates the hypothesis and the experimental results as well as confirms that the computational simulations contain the appropriate physics models.

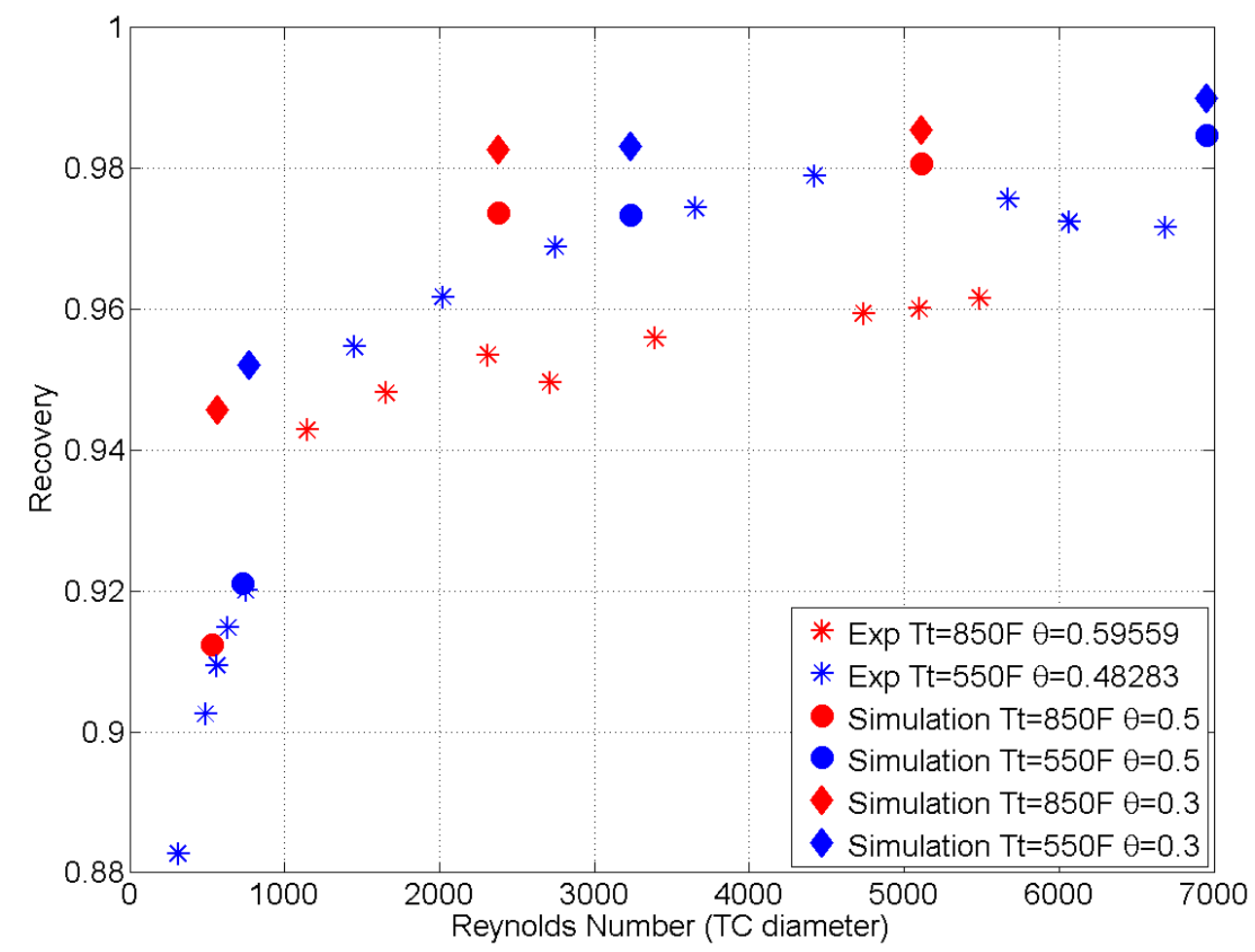

Figure 34: Recovery as a Function of Reynolds Number for Conduction Error Probe Experimental Data [14] and Current Predictions

\subsubsection{Convective Heat Transfer Coefficient Results}

It is useful to investigate the convective heat transfer coefficients, because the recovery is a direct function of it. Since this is not easily done experimentally, this was an ideal opportunity to apply the results of the computational simulations. The convective heat transfer coefficients were calculated from the simulations using Newton's Law of 
Cooling seen below. In this formulation, the reference temperature was chosen to be the value at a point in uniform flow within the shield. This point was chosen because it represented the mean temperature throughout the shield which would drive the convective heat transfer process. The reference point can be seen in Figure 35.

$$
q^{\prime \prime}{ }_{w}=h\left(T-T_{r e f}\right)
$$

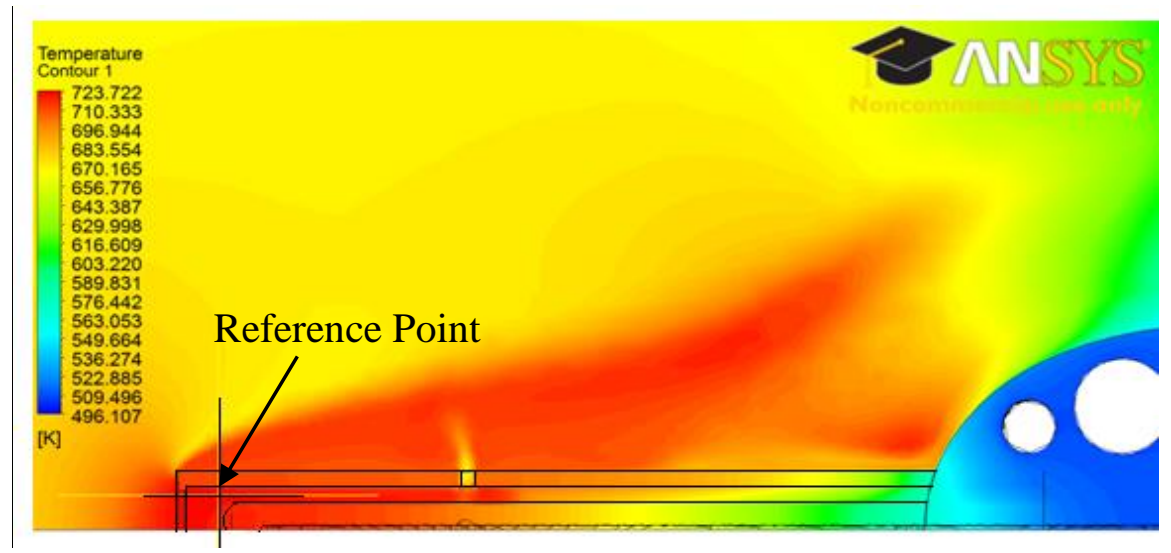

Figure 35: In-Shield Reference Point used in Heat Transfer Coefficient Calculations

Traditionally, the heat transfer coefficients are presented using non-dimensional numbers like the Nusselt Number as defined in Equations 27. Because the heat transfer over the thermocouple was being investigated, the thermocouple diameter was chosen as the length scale for defining the Nusselt number. Also, because the heat transfer process takes place inside the shield, the thermo-physical properties were evaluated at the same point as the reference temperature. This is in contrast to the Reynolds number used in Figure 34 to relate the recovery to the flow field. The reason for this change was that the Reynolds number used in Figure 34 was chosen because it could be easily calculated with known flow quantities and therefore could be used to predict the recovery a priori. However, because the heat transfer to the thermocouple is directly dependent on the local flow, it was necessary to use the local properties in the investigation of the heat transfer coefficients. It should also be noted that the temperature at the reference point inside the shield was very close to, but were not exactly the same as the stagnation values of the flow properties. This suggests that if it was desired to predict these quantities a priori as was done with the recovery, one could use the total temperature in formulation of the Reynolds number.

$$
N u=\frac{h d_{T C}}{k_{f}}
$$

Typical contours of the wall heat flux, and Nusselt number on the surface of the thermocouple sheath are shown in Figures 36 and 37 for the case of total temperature of $850^{\circ} \mathrm{F}$, Mach number of 0.8 , and conduction driver of 0.5 . Note that the wall heat flux is negative indicating heat flow is from the fluid domain into the solid domain. Also, significant heat transfer due to convection only exists up to the vent holes. This is due to the stagnated flow that exists inside the shield downstream of the vent holes. 


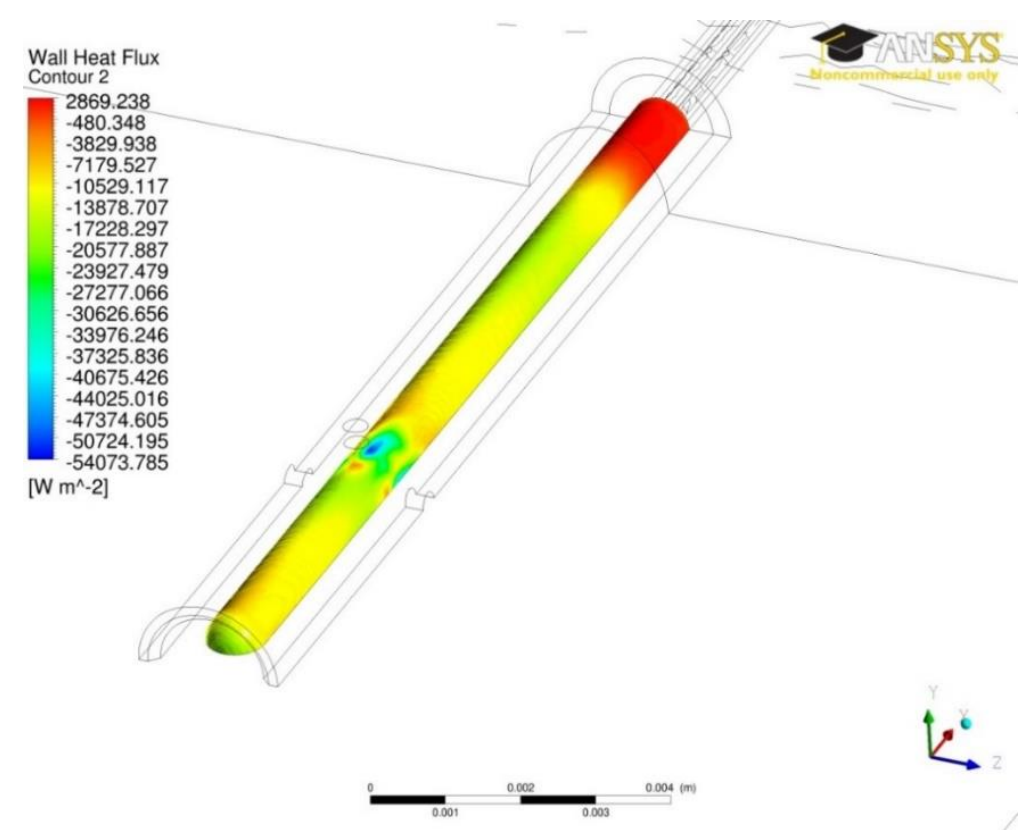

Figure 36: Contours of Wall Heat Flux along Thermocouple Body for Conduction Error Probe at Total Temperature of $850^{\circ} \mathrm{F}(727 \mathrm{~K})$, Mach Number of 0.8 , and Conduction Driver of 0.5

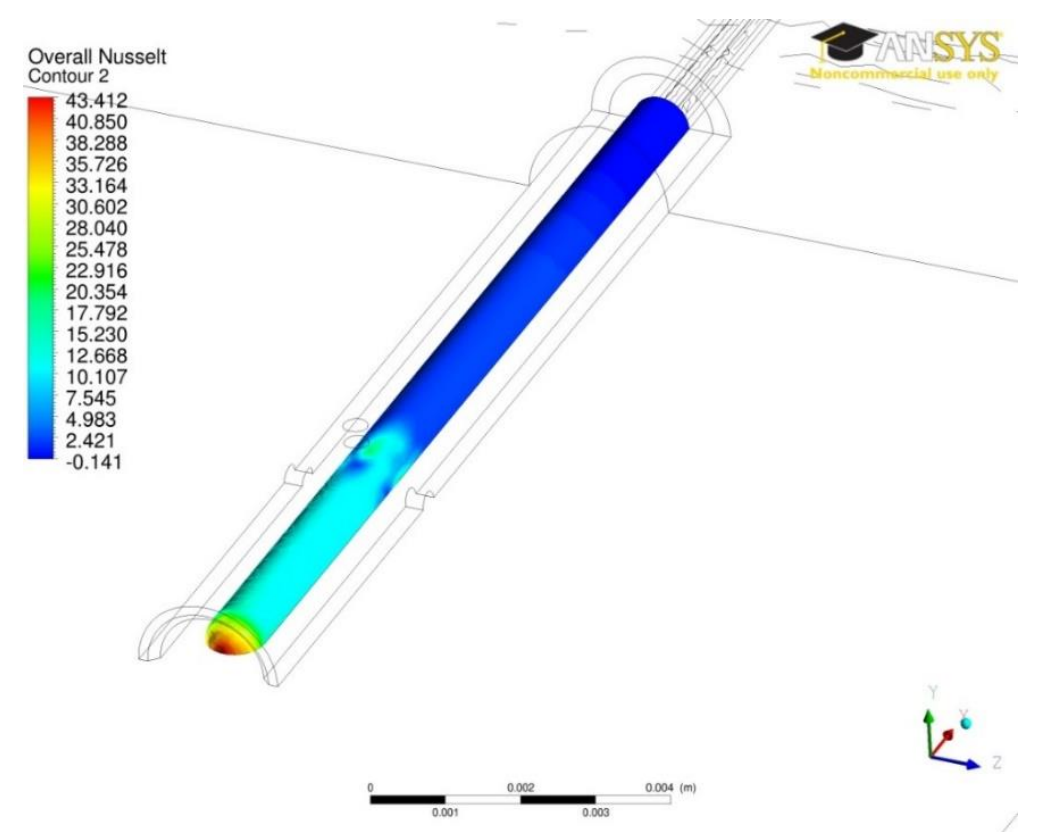

Figure 37: Contours of Nusselt Number along Thermocouple Body for Conduction Error Probe at Total Temperature of $850^{\circ} \mathrm{F}(727 \mathrm{~K})$, Mach Number of 0.8 , and Conduction Driver of 0.5

At the stagnation point, the heat transfer, and therefore the heat transfer coefficient, is much higher than along the length of the thermocouple, as expected. In the literature, the ratio of the heat transfer coefficient at the stagnation point to that along the sides of a hemisphere cylinder has been found to be about 3-5. To compare with this rule of thumb, 
the maximum heat transfer coefficient from the stagnation point was compared with an average value along the side of the thermocouple. The average only included the region immediately after the end of the radius of the TC to the end of the uniform region in front of the vent holes. Tabulated values for the ratio of the heat transfer coefficient at the stagnation point to the average side value in each case simulated are given in Table 9. For all of the cases simulated, the maximum heat transfer coefficient at the stagnation point was indeed between 3 to 5 times the average values along the body of the thermocouple. This was an important finding as it was used in the development of extended lower-order analysis techniques. Specifically, a fin analysis assuming one-dimensional heat transfer was being developed. This model initially used only a single average heat transfer coefficient with an adiabatic tip condition. However, this has now been modified to include separate heat transfer coefficients for the tip and the sides of the fin.

Table 9: Ratio of Convective Heat Transfer Coefficient at Stagnation Point to Average along Body of Thermocouple

\begin{tabular}{|c|c|c|c|}
\hline \multicolumn{3}{|c|}{ Simulated Case } & \multirow{2}{*}{$\begin{array}{l}\text { Film Coefficient } \\
\text { Ratio }\left(\mathbf{h}_{\text {tip }} / \mathbf{h}_{\text {side }}\right)\end{array}$} \\
\hline $\begin{array}{c}\text { Total Temperature } \\
\left({ }^{\circ} \mathbf{F}\right)\end{array}$ & $\boldsymbol{\theta}$ & $\mathbf{M}$ & \\
\hline \multirow{3}{*}{850} & \multirow{3}{*}{0.5} & 0.8 & 3.5 \\
\hline & & 0.4 & 3 \\
\hline & & 0.1 & 4.8 \\
\hline \multirow{3}{*}{850} & \multirow{3}{*}{0.3} & 0.8 & 3.6 \\
\hline & & 0.4 & 3 \\
\hline & & 0.1 & 4.7 \\
\hline \multirow{3}{*}{550} & \multirow{3}{*}{0.5} & 0.8 & 3.8 \\
\hline & & 0.4 & 3.3 \\
\hline & & 0.1 & 3.7 \\
\hline \multirow{3}{*}{550} & \multirow{3}{*}{0.3} & 0.8 & 4 \\
\hline & & 0.4 & 3.3 \\
\hline & & 0.1 & 3.6 \\
\hline
\end{tabular}

Also, in a legacy paper, Moffat [9] presented a correlation of the Nusselt Number as a function of the Reynolds number for bare-wire thermocouples parallel and perpendicular to the flow shown in Equations 28 and 29, respectively. These correlations were also used extensively in simple analyses and it was desired to compare the results of the computational simulations with these correlations.

$$
N u=(0.085 \pm 0.009) R e^{0.674}
$$




$$
N u=(0.44 \pm 0.06) R e^{0.5}
$$

A comparison of the Nusselt Numbers from Moffat's correlation for flow parallel to a thermocouple wire and those obtained from the simulations is given in Figure 38. The Nusselt number values from the simulations are obtained using the flow properties evaluated at the reference point shown in Figure 35 and the diameter of the thermocouple as the characteristic length scale. The values presented are the maximum values at the stagnation point. The Nusselt numbers from Moffat's correlation use the total temperature of the flow to evaluate the properties and the bare-wire thermocouple bead diameter as the characteristic length. To compare the results with Moffat's correlation, the Reynolds number was calculated using the freestream velocity and evaluating the gas properties at the total temperature. It can be seen that the results obtained computationally compare qualitatively well with the legacy correlation and certainly follow the same trend. It is important to note however, that Moffat's correlation was for bare-wire thermocouples without a shield as opposed to the shielded, sheathed thermocouple that was simulated. Using the same functional form as the correlation due to Moffat, a correlation based on the computational results can be given as $N u=0.5897 R e^{0.4857}$.

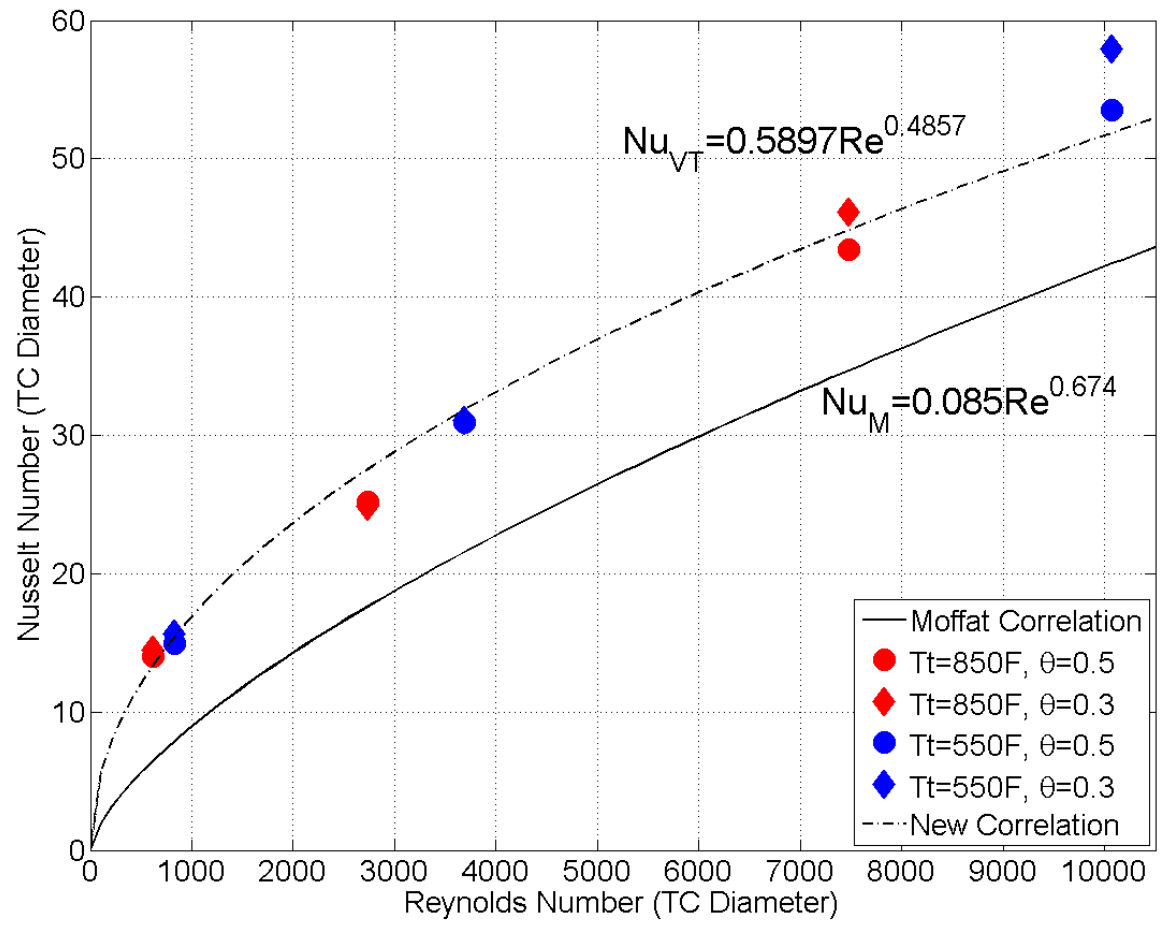

Figure 38: Comparison of Average Max Nusselt Numbers from Simulations and Legacy Correlation from Ref. 9

The Reynolds numbers used in plotting the Nusselt numbers of Figure 38 were based on the freestream velocity because this is what Moffat's correlation used (since he was analyzing bare-wire thermocouples). However, this is obviously not the velocity inside the shield. Moffat [9] does suggest a general guideline for flow through shielded sensors: the internal velocity is approximately $1 / 8^{\text {th }}$ the external velocity for a probe with a vent- 
to-inlet area ratio of about $20 \%$. It was, therefore, desired to compare the computational results with this general guideline to assess its applicability. The ratio of the in-tube velocity and the freestream velocity from the computational simulations are plotted below in Figure 39. As can be seen, Moffat's general rule is not a bad approximation, although it seems to underestimate the velocity for the geometry of the current sensor.

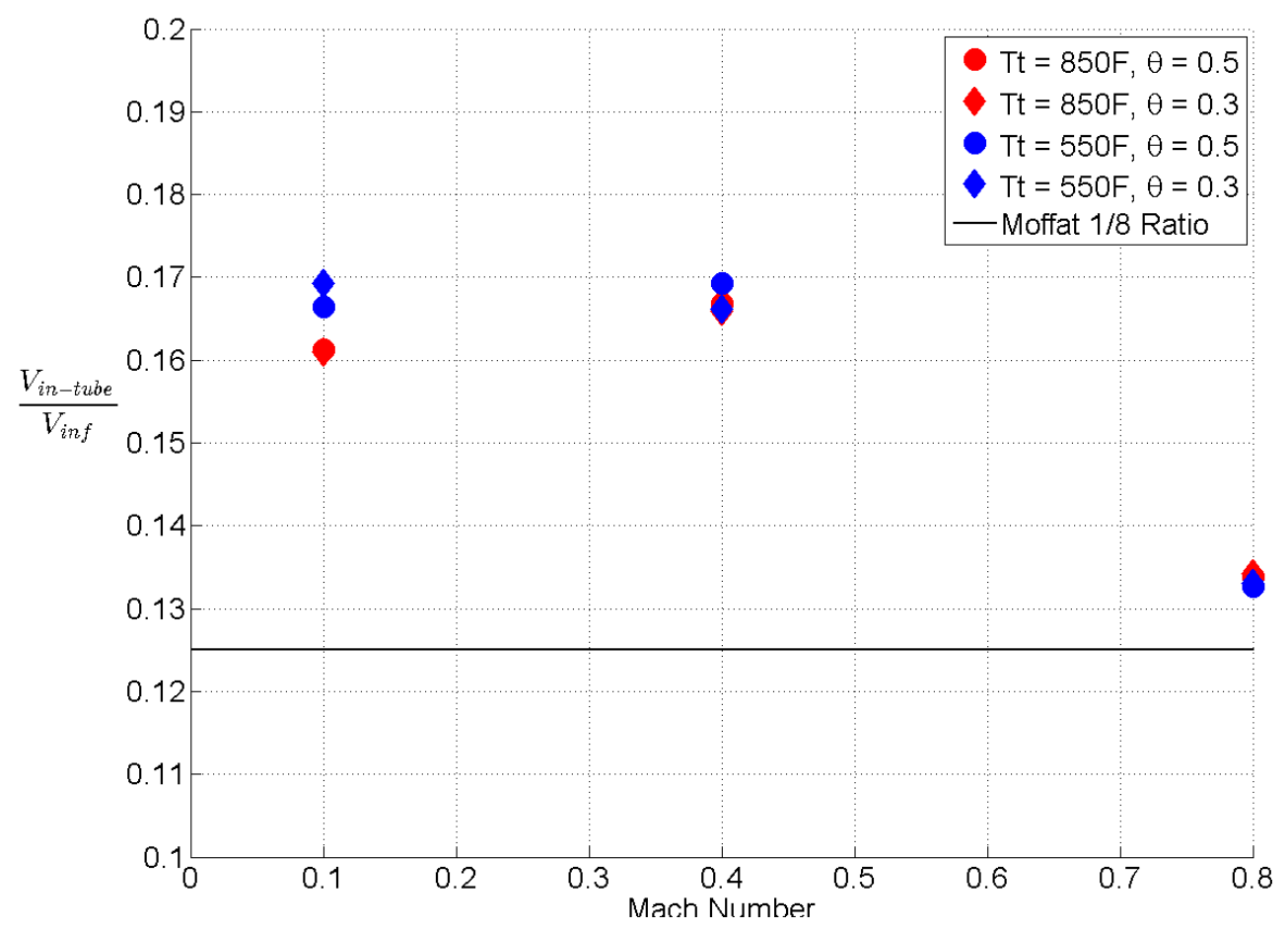

Figure 39: Ratio of In-Tube Velocity to Freestream Velocity for CFD Predictions and Estimate from Ref. 9

\subsubsection{Flow Field Results}

Although much interest has been placed on the heat transfer processes in the simulations, it is important to analyze the flow that exists over the thermocouple-strut assembly as the convective heat transfer is a direct result of this flow. In particular, it was found that the strut greatly affected the flow over the probe. Contours of the Mach number throughout the domain are shown in Figure 40 below for the case with a total temperature of $850^{\circ} \mathrm{F}$, Mach number of 0.8 , and conduction driver of 0.5 . As can be seen, as the flow encounters the strut it is accelerated over it and even reaches low supersonic Mach numbers (for the results shown with a relatively high subsonic Mach number). Also, in particular, notice the area of separated flow from the shield due to the adverse pressure gradient created by the presence of the strut seen in Figure 41. The flow exiting the vent holes is actually deflected forward by the presence of the strut. This is even more evident in Figure 42 which shows the streamlines entering the shield and exiting the vent holes. In this figure, the streamlines are colored by the velocity. The flow exiting the vent holes is greatly influenced by the presence of the strut and creates a complex vortical flow pattern. This can have a significant impact on the internal flow and heat transfer to the thermocouple. 
Also, this vortical flow may lead to unsteady flows in and around the shield which could lead to structural failure or fatigue of the thermocouple that would limit its operational lifetime. Finally, it is also important to point out that the trailing edge of the strut was blunted, which also led to a large separation region. This wake region is characterized by large amounts of turbulent mixing and therefore increased heat transfer. Therefore, the trailing edge is being significantly heated which can be significant at higher temperatures.

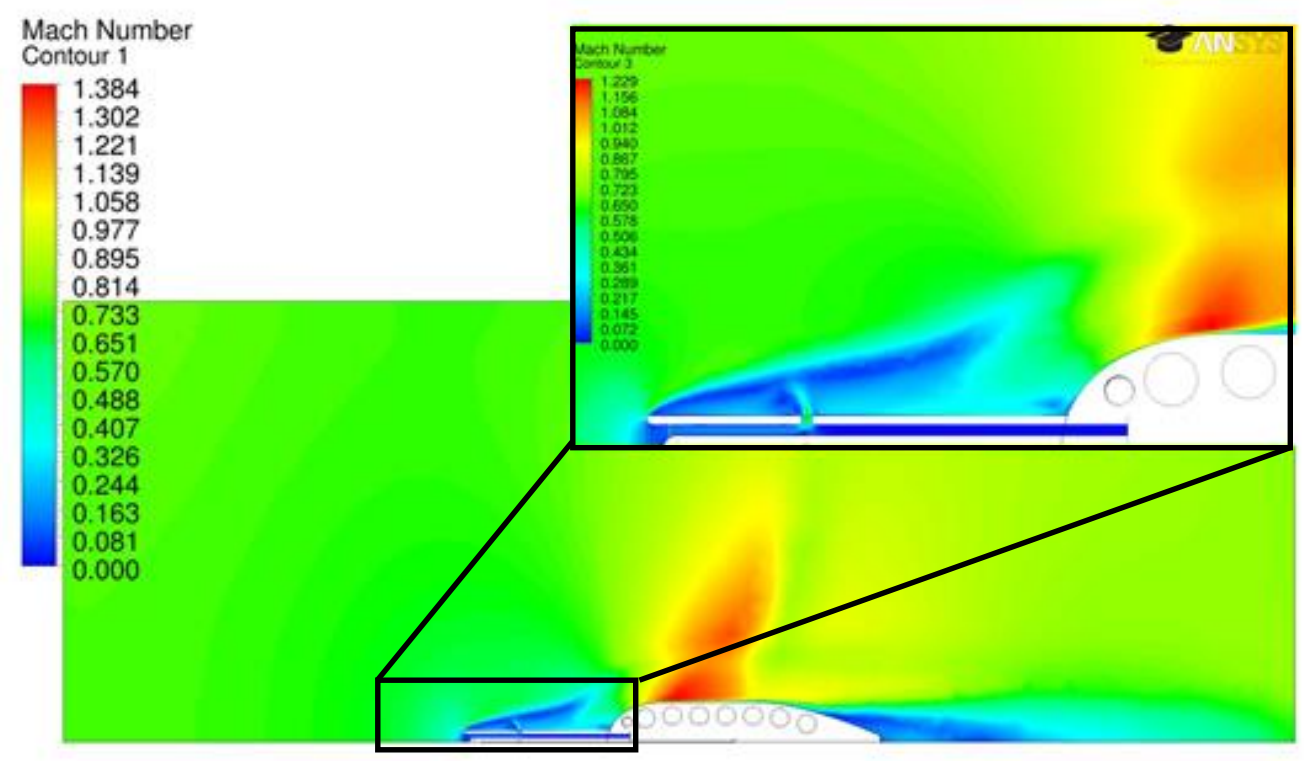

Figure 40: Mach Number Contours for Conduction Error Probe at Total Temperature of $850^{\circ} \mathrm{F}(727 \mathrm{~K})$, Mach Number of 0.8 , and Conduction Driver of 0.5

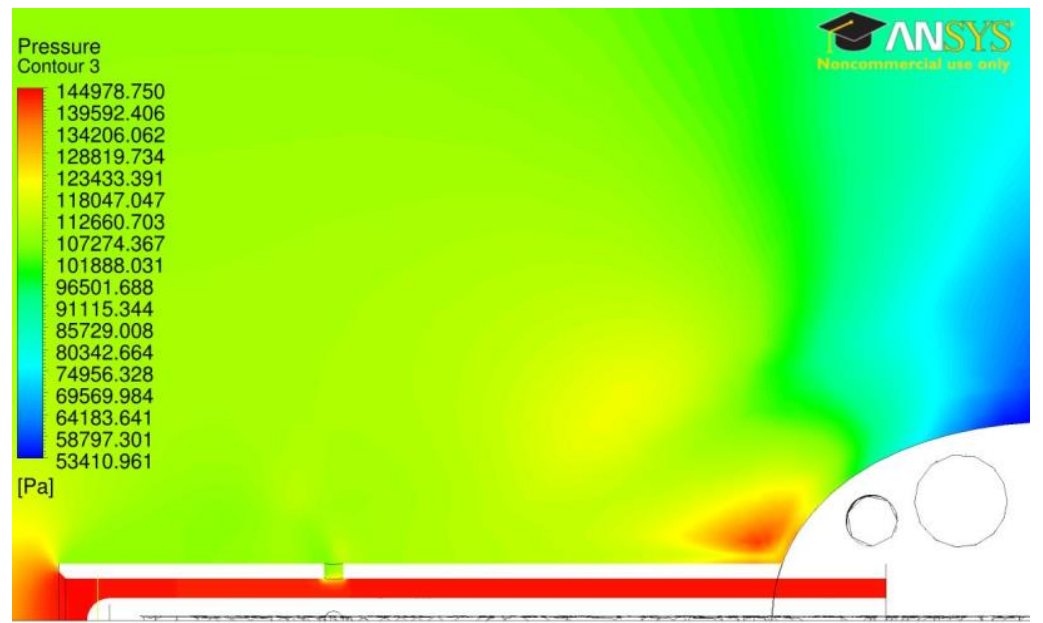

Figure 41: Pressure Contours at Leading Edge of Strut for Conduction Error Probe at Total Temperature of $850^{\circ} \mathrm{F}(727 \mathrm{~K})$, Mach Number of 0.8 , and Conduction Driver of 0.5 


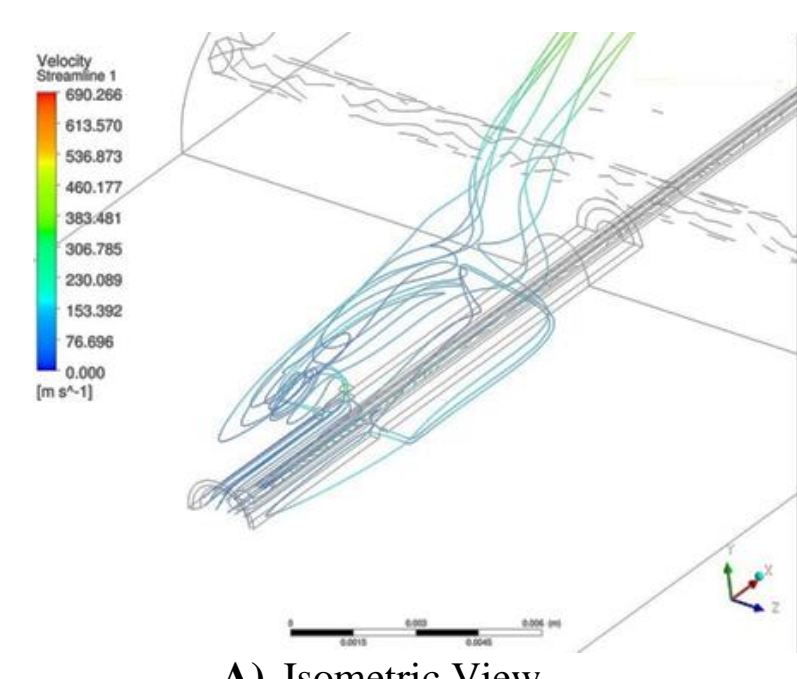

A) Isometric View

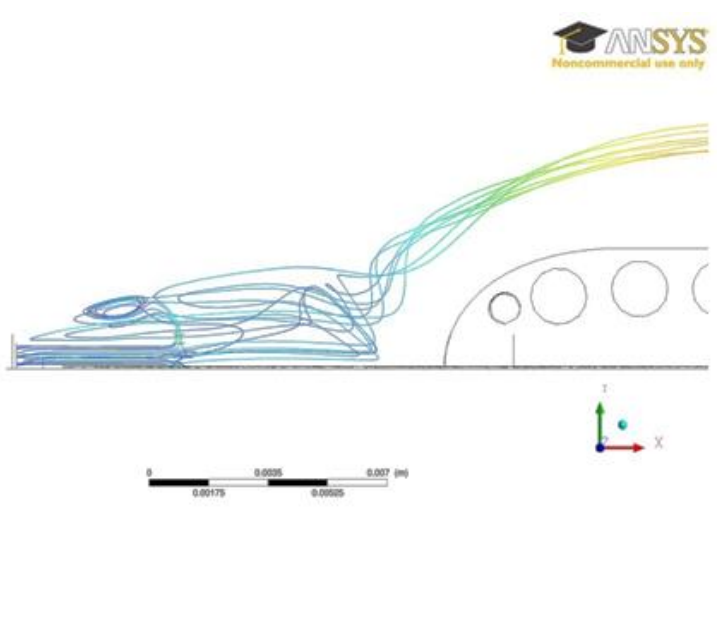

B) Side View

Figure 42: Streamlines through Shield for Conduction Error Probe at Total Temperature of $850^{\circ} \mathrm{F}(727 \mathrm{~K})$, Mach Number of 0.8 , and Conduction Driver of 0.5

\subsubsection{Basic Uncertainty Study}

Finally, because there were large uncertainties in some of the modeling parameters for the radiation calculations, it was desired to estimate the effect of these uncertainties on the results. To do this, an uncertainty analysis was conducted using the root-sum-square method for the radiation correction presented by Moffat [9]. Moffat's radiation correction is based on the balance of convective and radiative heat transfer and is shown in Equation 2. It should be noted that this radiation correction will be a worst case scenario as it does not take into account the effect that the shield has on the radiation heat transfer.

To obtain the uncertainty, it was assumed that the radiation correction is a function of four variables, specifically, the emissivity, the junction temperature, the surrounding temperature, and the convective heat transfer coefficient. The proceeding development follows from uncertainties in measurements as presented in Doeblin [1]. The radiation correction can be written in functional form such that $E_{r a d}=f\left(\varepsilon_{w}, T_{j}, T_{t}, h_{c}\right)$. If a small inaccuracy in the measurement or calculation of theses variables is assumed, a Taylor's series expansion can be used to approximate the change in the radiation correction for the corresponding inaccuracy such that

$$
\Delta E_{\text {rad }}=\frac{\partial f}{\partial \varepsilon_{w}} \Delta \varepsilon_{w}+\frac{\partial f}{\partial T_{j}} \Delta T_{j}+\frac{\partial f}{\partial T_{\text {surr }}} \Delta T_{\text {surr }}+\frac{\partial f}{\partial h_{c}} \Delta h_{c}
$$

As is done for measuring devices, the $\Delta$-terms can be interpreted as uncertainties so that the overall uncertainty in the radiation correction is given as the root sum square. It should be noted that the partial derivatives show the sensitivity of the radiation correction with respect to changes in the corresponding variable and that they are constants, evaluated at the operating condition. 


$$
\delta E_{\text {rad }}=\sqrt{\left(\frac{\partial f}{\partial \varepsilon_{w}} \delta \varepsilon_{w}\right)^{2}+\left(\frac{\partial f}{\partial T_{j}} \delta T_{j}\right)^{2}+\left(\frac{\partial f}{\partial T_{\text {surr }}} \delta T_{\text {surr }}\right)^{2}+\left(\frac{\partial f}{\partial h_{c}} \delta h_{c}\right)^{2}}
$$

To apply this model, one must have measured or estimated values for the input variables, namely $\varepsilon_{w}, T_{j}, T_{\text {surr }}$ and $h_{c}$. This is easily done if computational simulations are already completed as these variables can be readily extracted. If an estimate is desired without running additional simulations, the desired variables can be found using correlations such as those presented for the Nusselt number in a previous section.

This procedure was applied to a case that was modeled in the previous section. Specifically, the case with the total temperature of $850^{\circ} \mathrm{F}$, Mach number of 0.8 , and conduction driver of 0.5 was used. The values for $\varepsilon_{w}, T_{j}, T_{\text {surr }}$ and $h_{c}$ are shown in Table 10. Again, these values were directly extrapolated from the computational results. Because this simulation included radiation effects, the uncertainty estimated using the above formulation will yield bounds on the accuracy of the simulation with respect to the four input variables. In the analysis, each variable was analyzed for an uncertainty ranging from $0-100 \%$ of the nominal value used while assuming the uncertainty in the other variables was 0 . The results of this can be seen in Table 11 which shows the sensitivity derivatives and Figure 43 which shows the overall uncertainty in the radiation correction.

Table 11 indicates that the radiation correction is most sensitive to the value of emissivity. However, because the emissivity can only range between zero and one, the actual uncertainty in the emissivity, $\delta \varepsilon_{w}$, is going to be small, as seen in Figure 43 . The next largest sensitivity derivative corresponds to the junction temperature $T_{j}$. This makes sense because it is the driving factor in the radiation heat transfer process.

Table 10: Input Variables for Radiation Uncertainty Quantification at Total Temperature of $850^{\circ} \mathrm{F}(727 \mathrm{~K})$, Mach Number of 0.8 , and Conduction Driver of 0.5

\begin{tabular}{|c|c|}
\hline Variable & Nominal Values \\
\hline \hline$T_{j}$ & $713.5 \mathrm{~K}$ \\
\hline$T_{\text {surr }}$ & $300 \mathrm{~K}$ \\
\hline$\varepsilon_{w}$ & 0.2 \\
\hline$h_{c}$ & $2858 \mathrm{~W} / \mathrm{m}^{2}-\mathrm{K}$ \\
\hline
\end{tabular}


Table 11: Sensitivity Derivatives for Radiation Uncertainty at Total Temperature of $850^{\circ} \mathrm{F}(727 \mathrm{~K})$, Mach Number of 0.8 , and Conduction Driver of 0.5

\begin{tabular}{|c|c|}
\hline Variable & Sensitivity Derivative \\
\hline$T_{j}$ & $5.75 \mathrm{E}-3$ \\
\hline$T_{\text {surr }}$ & $-4.28 \mathrm{E}-4$ \\
\hline$\varepsilon_{w}$ & 4.981 \\
\hline$h_{c}$ & $-3.48 \mathrm{E}-4$ \\
\hline
\end{tabular}
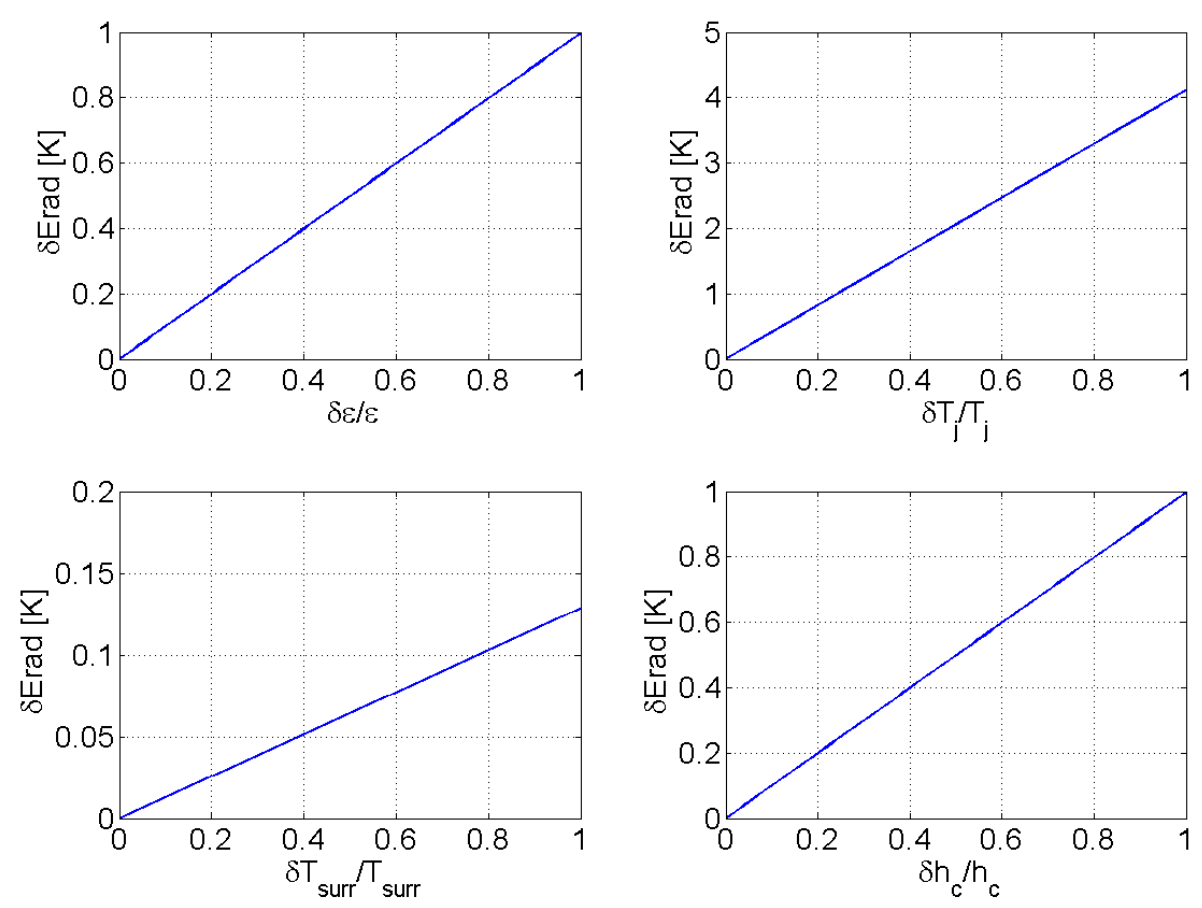

Figure 43: Uncertainty in Radiation Correction for Four Variables of Interest for Conduction Error Probe at Total Temperature of $850^{\circ} \mathrm{F}(727 \mathrm{~K})$, Mach Number of 0.8 , and Conduction Driver of 0.5

Now looking specifically at the uncertainty in the radiation correction given in Figure 43, the error in the radiation correction increases linearly with an increase in uncertainty of the individual variables. This is because each is being considered separately with the other uncertainties set at zero. As can be seen, at this low temperature, there are only small errors in the radiation correction even for large uncertainties in the given variable. At these conditions, the effect of radiation is least for changes in the surrounding temperature which perhaps had the largest uncertainty in the computational simulations. Also, another source of uncertainty in the simulations was the value of emissivity. However, it can be seen that even if the emissivity was $100 \%$ off, then the radiation correction is only about $1 \mathrm{~K}$ different than if the emissivity was known with $100 \%$ certainty. The variable that causes the largest uncertainty is the junction temperature. However, in reality, this uncertainty should be quite small because it is in fact what is being obtained computationally, thus plotting the error to $100 \%$ is truly only academic. 


\section{Discussion}

A brief discussion of the modeling assumptions and implications will be given first. Then, the lessons learned from radiation modeling and the overall usefulness of computer simulations in the design process will be highlighted.

\subsection{Comments on Modeling Assumptions}

First, because the computational domain can never include all the details of the surroundings, it was necessary to consider the effects that radiative heat transfer to and from the surroundings had on sensor performance. For example, in the validation case, it was found that radiation from the flame in the combustor had an effect, because the thermocouple had a direct view factor to it. The same can be said in the conduction test error case with respect to the upstream heating elements and flow control devices which were again directly upstream of the sensor. Although this may have had a relatively minor effect in that work, in other studies this may not be small and therefore considering all radiation sources and sinks is imperative. In addition to the warmer surfaces upstream, the cooler surroundings were of more importance. This is because the surroundings consisted of a much larger portion of the total solid angle that the probe could "see". Of course, the surroundings at temperatures lower than the surface of the thermocouple would cause the radiative cooling effect and lead to the radiation error. However, it was interesting to note that the warmer surfaces such as the flame or flow straightener would tend to help reduce this error at least to some small degree.

It should also be noted that there was uncertainty in choosing accurate values of emissivity for the different surfaces. In fact, the use of a constant surface emissivity itself was a limiting assumption. This technically is not the case because even at the relatively low temperatures that some of the experimental tests were conducted at, it could be seen that the surfaces of the materials became darker with use, thus indicating that the emissivity was increasing. However, the only way to know definitively the emissivity of the surfaces would be to measure them experimentally which would require extensive work.

Also, it is important to reconsider several assumptions that were made during the modeling processes to reduce the time and resources needed for the computational simulations. First and most importantly was the use of symmetric models. By simulating only half of the model, the computational domain could be refined much more than if a full three dimensional model was made with a comparable number of elements. This of course is a tradeoff; while the simulation may be more accurate due to a more refined mesh, the ability to compute any asymmetric flow field is lost, for example pitch or yaw angles. However, this study did not investigate the orientation of the sensors so the symmetric model was used.

Finally, the modeling conduction error due to the cooling channels was simplified. First it was assumed and then it was proven that actually modeling the flow through these channels was unnecessary. Instead, specification of a wall temperature to yield the 
desired base temperature for the conduction driver was sufficient. This is not insignificant as modeling the flow through these channels would have substantially increased the size of the mesh which would then have increased the time needed to converge the simulation. This increase in time and resources would not be warranted as no important information would be gained by the added complexity.

\subsection{Comments on Turbulence Model}

Throughout this work, the $k-\omega$ SST turbulence model was used. However, it is interesting to note that the eddy viscosity inside the shield is quite low in most cases. This suggests that the turbulence model may not be significant in calculating the heat transfer to the thermocouple inside of the shield. For example, Table 12 shows the eddy viscosity ratio, defined as the ratio of the eddy viscosity to the laminar viscosity.

Table 12: Eddy Viscosity Ratio inside Shield of Conduction Error Probe

\begin{tabular}{|c|c|c|c|}
\hline \multicolumn{3}{|c|}{ Simulated Case } & \multirow{2}{*}{$\begin{array}{l}\text { Eddy Viscosity } \\
\text { Ratio }\left(\mu_{T} / \mu\right)\end{array}$} \\
\hline $\begin{array}{c}\text { Total Temperature } \\
\left({ }^{\circ} \mathbf{F}\right)\end{array}$ & $\boldsymbol{\theta}$ & $\mathbf{M}$ & \\
\hline \multirow{3}{*}{850} & \multirow{3}{*}{0.5} & 0.8 & 1.38 \\
\hline & & 0.4 & 0.483 \\
\hline & & 0.1 & 0.0148 \\
\hline \multirow{3}{*}{850} & \multirow{3}{*}{0.3} & 0.8 & 1.39 \\
\hline & & 0.4 & 0.483 \\
\hline & & 0.1 & 0.0175 \\
\hline \multirow{3}{*}{550} & \multirow{3}{*}{0.5} & 0.8 & 1.699 \\
\hline & & 0.4 & 0.695 \\
\hline & & 0.1 & 0.0346 \\
\hline \multirow{3}{*}{550} & \multirow{3}{*}{0.3} & 0.8 & 1.698 \\
\hline & & 0.4 & 0.696 \\
\hline & & 0.1 & 0.0418 \\
\hline
\end{tabular}

The eddy viscosity ratio is very low inside of the shield but increases with Mach number. A low eddy viscosity ratio indicates that the eddy viscosity has a small effect compared to the laminar viscosity. Although the turbulent eddy viscosity plays an increasing role as the Mach number increases, it is always on the order of the laminar viscosity or below that. Therefore, the laminar processes will be more important than the turbulent 
processes, thus suggesting that the choice of the turbulence model is not critical and will not lead to large discrepancies.

It should be noted then, that as the diffusion due to viscosity is decreased, the numerical diffusion due to the grid will play a more important role. However, it is noted in Ref. 23 for example, that the numerical diffusion can be reduced by refining the mesh. That is exactly what was done in the vicinity surrounding the shield. The manual refinement region that was created should therefore reduce the effects of numerical diffusion and accurately calculate the heat transfer processes in the shield to the thermocouple.

\subsection{Comments on the Usefulness of Computational Simulations}

It is believed that this work highlights the advantages of including computational studies in the design and analysis of total temperature probes. The wide range of parameters that can be varied would require extensive laboratory testing. Also, quantities like the heat transfer coefficients can be obtained computationally more readily than in experimental work. Also, the effect of the airfoil strut mount on the external flow and internal flow exiting the vent holes was discovered through these computational simulations. The usefulness of simulations will continue to increase in future as temperature probe designs become smaller and smaller to be less intrusive in engine testing. Finally, computational validation is important in that it confirms that all the applicable physical phenomena are being considered. That is, in the setup of the simulations, different models need to be applied for different physical processes. If the results of the model do not compare well with the physical, experimental results, the question arises whether all the correct models are being applied. This can lead to further scrutiny of the physics and therefore lead to better understanding of the entire experiment.

\section{Conclusions and future work}

Overall, this work sought to use multi-physics computational simulations to model the performance of total temperature probes. The simulations developed were an extension of the methods developed in Ref. 15 to include the effects of radiation which become increasingly important at high temperatures. First a validation case was conducted where the computational methods were applied to data from legacy NACA probe designs. Specifically, the radiation correction over a range of total temperatures from 1600R to 2500R were calculated and compared to the experimental results documented in Ref. 10. Simulations were conducted using a three-dimensional, symmetric model and twodimensional, axisymmetric models. Despite several simplifications made to the geometry during the modeling process, good agreement was found for the radiation correction in these cases. This served as a validation of the computational method of procedure.

After successfully validating the methods, the simulations were used to model an experiment performed at Virginia Tech that focused on conduction errors. In this experiment, the goal was to isolate the effects of conduction error. The computational simulations were therefore used to assess the effect that radiation had on the experimental results. A three-dimensional symmetric model was created and in this case, it was found 
that the heat transfer due to radiation was negligible. Also, the recovery factor, the main performance parameter being investigated, was compared between the simulations and the experimental results. Very good agreement was found. In modeling this case, the computational results were used to investigate flow features that are not easily quantified experimentally. Specifically the heat transfer coefficients and the flow through the vented shield were investigated. The heat transfer coefficients were tabulated as Nusselt numbers and were compared to a legacy correlation. It was found that although the correlation under-predicted the Nusselt number, the predicted results did follow the same trend. A new correlation of the same functional form was therefore suggested. Also, it was confirmed that the heat transfer coefficient at the stagnation point on the thermocouple was three to five times higher than that along the length of the thermocouple which had been suggested in the literature. These correlations were in turn used in the development of analytical models.

Finally, it should be noted that although there were several simplifying assumptions made in the modeling process, good agreement with the physical data was found. Most notably were the use of symmetric models and the fact that instead of modeling the coolant flow through the cooling channels, a constant temperature was specified. These simplifications drastically reduced the computational cost needed yet still allowed for good comparison with the experimental work. This shows that the use of computational simulations during the design process can be an invaluable asset.

\subsection{Suggestions for Future Work}

Now that computational simulations have been shown to be useful in analyzing total temperature probes, more work can be done. For example, transient simulations to study the thermal time constants can be done. This can be easily implemented in the current simulations, but may require large computational time and resources. Also, full threedimensional models can be developed to assess the ability to computationally predict the effect of sensor orientation, specifically pitch and yaw angles. Again, because full threedimensional models would be needed, computational time and resources would be larger than in the current work.

Finally, and perhaps most importantly would be to extend the simulations to predict the sensor performance at much higher temperatures that are indicative of what they would experience in a true jet engine environment. At these temperatures other effects such as thermal-structural fatigue becomes prominent. Therefore, coupled fluid-thermalstructural simulations would be needed to accurately predict the performance of a total temperature sensor. In any event, the groundwork has been laid to pursue such work. 


\section{References}

[1] Doeblin, E.O. Measurement Systems: Application and Design $3^{\text {rd }}$ Ed. McGraw-Hill, New York, NY 1983

[2] Childs, Peter R.N. Practical Temperature Measurement. Butterworth-Heinemann, Oxford, 2001.

[3] Benedict, Robert P. Fundamentals of Temperature, Pressure, and Flow Measurements. Wiley, New York, 1984.

[4] Gerhard, R. E., "Shielded Thermocouples", In Physical Measurements in Gas Dynamics and Combustion, Princeton University Press, Princeton, NJ, 1954.

[5] Goldstein, R., Fluid Mechanics Measurements. CRC Press, 1996.

[6] Franz, A., "Pressure and Temperature Measurement in Supercharger Investigations", NACA Technical Report NACA-TM-953, National Advisory Committee for Aeronautics, 1940.

[7] Hottel, H.C., and Kalitinsky, A., “Temperature Measurements in High-Velocity Air Streams", Journal of Applied Mechanics, 12:A25-A32, 1945.

[8] Lindsey, W.F., "Calibration of Three Temperature Probes and Pressure Probe at High Speeds”, Report, National Advisory Committee for Aeronautics, 1942.

[9] Moffat, R.J., “Gas Temperature Measurement”, Temperature, Its Measurement and Control in Science and Industry, Vol. 3, Part 2, 1962.

[10] Glawe, G.E, Simmons, F.S., and Stickney, T.M., "Radiation and Recovery Corrections and Time Constants of Several Chromel-Alumel Thermocouple Probes in High Temperature, High Velocity as Streams", NACA TN 3766, National Advisory Committee for Aeronautics, 1956.

[11] King, W.J., "Measurement of High Temperatures in High-Velocity Gas Streams", Trans. ASME 65, 421, 1943.

[12] Reardon, J.P, Schneider, A., Schetz, J.A., and Lowe, K.T., Computational Modeling of Radiation Effects on Total Temperature Probes. $31^{\text {st }}$ AIAA Aerodynamic Measurement Technology and Ground Testing Conference, Dallas, TX, 2015.

[13] Scadron, M.D., and Warshawsky, I., "Experimental Determination of Time Constants and Nusselt Numbers for Bare-Wire Thermocouples in High-Velocity Air Streams and Analytic Approximation of Conduction and Radiation Errors", NACA TN 2599, National Advisory Committee for Aeronautics, 1952. 
[14] S.T. Englerth, "An Experimental Conduction Error Calibration Procedure for Cooled Total Temperature Probes", MS Thesis, Aerospace and Ocean Engineering Department, Virginia Polytechnic Institute \& State University, Blacksburg, VA, May 2015.

[15] Schneider, A., "Computational Modeling of Total Temperature Probes", MS Thesis, Aerospace and Ocean Engineering Department, Virginia Polytechnic Institute \& State University, Blacksburg, VA, May 2015.

[16] Cebeci, T. Shao, J.P., Kafyeke, F., and Laurendeau, E. Computational Fluid Dynamics for Engineers: From Panel to Navier-Stokes Methods with Computer Programs. Long Beach, CA: Horizons Pub., 2005.

[17] Schetz, J.A., and Bowersox, R.D.W., Boundary Layer Analysis, $2^{\text {nd }}$ ed., AIAA Educational Series., AIAA Reston, VA, 2011.

[18] Pope, S.B., Turbulent Flows, Cambridge University Press, Cambridge, 2000.

[19] Moin, P. and Mahesh, K. "Direct Numerical Simulation: A Tool in Turbulence Research". Annual Review of Fluid Mechanics, Vol 30 pp 539-578. 1998.

[20] Cebeci, T. Turbulence Models and Their Applications. Horizons Publishing Inc., Long Beach, CA 2004.

[21] Spalart, P.R. and Allmaras, S.R. "A One-Equation Turbulence Model for Aerodynamic Flows". AIAA Paper 92-0439, 1992.

[22] Jones, W.P. and Launders, B.E. "The Prediction of Laminarization with a TwoEquation Model of Turbulence". Int. J. Heat and Mass Transfer 15, 301-314, 1972.

[23] ANSYS Inc. ANSYS FLUENT, Release 14.5, Help System.

[24] Durbin, P.A. "Applications of a Near-Wall Turbulence Model to Boundary Layers and Heat Transfer". International Journal of Heat and Fluid Flow, Vol 14, No. 4, pp 316-323. 1993.

[25] Simpson, R.L., Whitten, D.G., and Moffat, R.J. "An Experimental Study of the Turbulent Prandtl Number of Air with Injection and Suction". International Journal of Heat and Mass Transfer, Vol. 13, pp 125-143. 1970.

[26] Bergman, Lavine, Incropera, and Dewitt. Fundamentals of Heat and Mass Transfer. John Wiley \& Sons. 2011.

[27] Glawe, G.E., and Shepard, C.E., "Some Effects of Exposure to Exhaust-Gas Streams on Emittance and Thermoelectric Power of Bare-Wire Platinum Rhodium-Platinum Thermocouples", NACA TN 3253, 1954. 
[28] Svehla, R.A., and McBride, B.J., "Fortran IV Computer Program for Calculation of Thermodynamic and Transport Properties of Complex Chemical Systems", NASA Technical Note TN D-7056.

[29] Greene, G.A., Finfrock, C.C., and Irvine, T.F., Jr. "Total Hemispherical Emissivity of Oxidized Inconel 718 in the Temperature Range $300-1000^{\circ} \mathrm{C}$ ", Experimental Thermal and Fluid Science 22, May, 2000, p145-153. 